\title{
A short perinuclear amphipathic $\alpha$-helix in Apq12 promotes nuclear pore complex biogenesis
}

3

4

5

6

Wanlu Zhang1, 3, 4, Azqa Khan ${ }^{1,4}$, Jlenia Vitale ${ }^{1}$, Annett Neuner ${ }^{1}$, Kerstin Rink ${ }^{2}$, Christian Lüchtenborg $^{2}$, Britta Brügger ${ }^{2}$, Thomas H. Söllner ${ }^{2}$, Elmar Schiebel ${ }^{1}$

1 Zentrum für Molekulare Biologie der Universität Heidelberg, DKFZ-ZMBH Allianz, Im Neuenheimer Feld 282, 69120 Heidelberg, Germany

2 Biochemie-Zentrum der Universität Heidelberg, Im Neuenheimer Feld 328, 69120 Heidelberg, Germany

${ }^{3}$ Present address: Cell Biology and Biophysics Unit, European Molecular Biology Laboratory (EMBL), Heidelberg, Germany

${ }^{4}$ both authors contributed equally

Correspondence to Elmar Schiebel: e.schiebel@zmbh.uni-heidelbeg.de ORCID ID of E. Schiebel: orcid.org/0000-0002-3683-247X 


\section{Summary}

22 The integral membrane protein Apq12 is an important nuclear envelope (NE)/ER modulator 23 that cooperates with the nuclear pore complex (NPC) biogenesis factors Brl1 and Brr6. How 24 Apq12 executes these functions is unknown. Here we identified a short amphipathic $\alpha$-helix $(\mathrm{AaH})$ in Apq12 that links the two transmembrane domains in the perinuclear space and has liposome-binding properties. Cells expressing an APQ12 (apq12-ah) version in which $\mathrm{A \alpha H}$ is disrupted show NPC biogenesis and NE integrity defects, without impacting upon Apq12-ah topology or NE/ER localization. Overexpression of APQ12 but not apq12-ah triggers striking over-proliferation of the outer nuclear membrane (ONM)/ER and promotes accumulation of phosphatidic acid (PA) at the NE. Apq12 and Apq12-ah both associate with NPC biogenesis intermediates and removal of $\mathrm{AaH}$ increases both $\mathrm{Brl} 1$ levels and the interaction between Brl1 and Brr6. We conclude that the short amphipathic a-helix of Apq12 regulates the function of Brl1 and Brr6 and promotes PA accumulation at the NE during NPC biogenesis. 


\section{Introduction}

The nuclear envelope (NE) is a double membrane consisting of the outer nuclear (ONM) and inner nuclear (INM) membranes that surround and protect the nucleus. The ONM is continuous with the endoplasmic reticulum (ER), contains attached ribosomes, carries attachment sites for cytoskeletal elements and shares components with the ER (reviewed in Hetzer et al., 2005). In addition, the ER and ONM are the sites of triacylglycerol (TAG) and steryl ester lipid biosynthesis (Sorger \& Daum, 2003). These lipids are essential for membrane growth, and, in case of TAG, also for energy storage in form of cytoplasmic lipid droplets (Hashemi \& Goodman, 2015, Thiam, Farese et al., 2013). The INM is involved in genome stability, chromatin organization and regulation of gene expression (Beck \& Hurt, 2017, Onischenko, Tang et al., 2017, Ungricht \& Kutay, 2017). A recent publication indicated that TAGs are also synthesised at the INM before being incorporated into nuclear lipid droplets (Romanauska \& Kohler, 2018). Consistent with these distinct functions, the INM and ONM are specified by proteins and lipids that are district from one another.

The NPC is a large oligomeric complex containing about 30 different proteins that is embedded at sites at which the INM and ONM fuse. The NPC acts as a gateway at the partition between the cytoplasm with the nucleoplasm (Beck \& Hurt, 2017). In human cells and other eukaryotes undergoing an open mitosis, NPCs assemble via two mechanistically distinct pathways. The post-mitotic pathway promotes the assembly of NPCs on the decondensing chromatin, shortly after anaphase onset (Otsuka, Steyer et al., 2018). During interphase, NPCs assemble by a distinct inside-out mechanism starting from within the nucleus at the INM, the so-called interphase pathway (Otsuka, Bui et al., 2016, Winey, Yarar et al., 1997, Zhang, Neuner et al., 2018). In budding yeast, with its closed mitosis, the interphase pathway is the only mechanism to assemble NPCs.

NPC biogenesis intermediates of the interphase pathway have been described in human cells by electron tomography (Otsuka et al., 2016). In wild type (WT) yeast cells, NPC intermediates have not been observed, probably because NPCs assembly is relatively fast and infrequent ( 2 assembly events per minute per cell (Winey et al., 1997)). However, mutations in genes coding for several nucleoporins (Nups) lead to the accumulation of, so called, herniations, deformations of the INM that are probably filled with Nups (Aitchison, Blobel et al., 1995, Murphy, Watkins et al., 1996, Rampello, Laudermilch et al., 2020, Wente \& Blobel, 1993). Recently, we suggested that at least some of these herniations arise from defective parts of the NPC biogenesis pathways, for example the failure of the fusion of the 
INM with the ONM during the assembly process (Onischenko et al., 2017, Zhang et al., 2018).

Mechanistic principles of NPC complex biogenesis by the interphase pathway are poorly understood. In mammalian cells, early factors involved in interphase NPC biogenesis include Y-complex subunits NUP107 and NUP133, the INM protein SUN1 and the transmembrane nucleoporin POM121 (Doucet, Talamas et al., 2010, Otsuka \& Ellenberg, 2018, Souquet, Freed et al., 2018, Talamas \& Hetzer, 2011). In yeast, the two paralogous integral NE proteins Brl1 and Brr6 function in NPC biogenesis (de Bruyn Kops \& Guthrie, 2001, Hodge, Choudhary et al., 2010, Lone, Atkinson et al., 2015, Saitoh, Ogawa et al., 2005, Tamm, Grallert et al., 2011, Zhang et al., 2018). Loss of function of Brl1 (Brr6 like protein No. 1) or Brr6 (bad response to refrigeration) gives rise to herniations without impacting upon the insertion or function of pre-existing NPCs to indicate that both proteins are specifically required for NPC assembly but not for the maintenance of assembled NPCs. Consistent with this notion, Brl1 and Brr6 only associate with NPC intermediates and not with fully assembled NPCs (Zhang et al., 2018). Brl1 and Brr6 physically and genetically interact with the small integral NE/ER protein Apq12 to reveal synergistic regulation by all three of these proteins (Hodge et al., 2010, Lone et al., 2015, Scarcelli, Hodge et al., 2007). Cells with a deletion in $A P Q 12(a p q 12 \Delta)$ are cold sensitive for growth for unknown reason. Puzzlingly, at $37^{\circ} \mathrm{C}$ apq12 12 cells show a defect in NPC biogenesis despite relatively normal growth (Thaller, Allegretti et al., 2019).

A clear functional link between $B R L 1$ and NPC assembly has been revealed by genetic suppression of the physical interaction between the FG repeat containing Nup116 and the inner ring protein Nup188 that has a scaffolding function during NPC biogenesis leading to the accumulation of herniations in NUP116 and NUP188 defective cells (Allegretti, Zimmerli et al., 2020, Onischenko et al., 2017). Interestingly, the INM/ONM fusion defect of these scaffolding defective yeast cells was efficiently suppressed by overexpression of $B R L 1$ but not of BRR6 (Zhang et al., 2018). This suggests a role of Brl1 in INM/ONM fusion and also shows that Brl1 and Brr6 have distinct functions during NPC biogenesis.

Here we report that Apq12 carries a short perinuclear amphipathic alpha-helix $(\mathrm{A \alpha H})$ connecting the two transmembrane domains. The $\mathrm{AaH}$ peptide binds to liposomes and amino acid substitutions that disrupt the amphipathic nature of the peptide abolish the binding to liposomes. Cells carrying an Apq12 with a defective AaH (apq12-ah) are cold sensitive for growth, show NPC biogenesis defects and disrupted NE even though the distribution of the mutant protein is unaffected and it assumes the correct topology within the 
108 membrane. Overexpression of $A P Q 12$ triggers strong over-proliferation of the ONM and the 109 accumulation of phosphatidic acid (PA) at the NE in a manner that is reliant upon the 110 presence of a functional $\mathrm{AaH}$. Apq12 associates with NPC biogenesis intermediates at bent 111 INM segments, a localization that does not require a functional $\mathrm{AaH}$. In addition, apq12-ah 112 mutant shows elevated Brl1 and Brr6 levels and enhanced interaction between Brl1 and 113 Brr6. Taken together, these data place the AaH of Apq12 into a strategic position to co114 ordinate PA accumulation at the NE, Brl1-Brr6 interaction and NPC biogenesis.

\section{Results}

\section{Apq12 carries a short lipid-binding AaH between the two transmembrane domains} Recently we have shown that the integral membrane protein Brl1 associates with NPC assembly intermediates and may promote fusion of the ONM with the INM during NPC biogenesis (Zhang et al., 2018). Brl1 interacts and cooperates with the integral membrane protein Apq12 (Lone et al., 2015). In order to gain an understanding of the molecular roles of Apq12 (Fig. 1A), we sought functional elements within this protein. The AmphipaSeeK program predicated an amphipathic $\alpha$-helix $(\mathrm{AaH})$ with two positively charged amino acids (Lys and Arg) on the hydrophilic side and hydrophobic amino acid residues on the opposite side of the helix (Fig. 1B; "Apq12") (Sapay, Guermeur et al., 2006), between the two TM domains of Apq12 (Fig. 1A).

The positively charged amino acids of an AaH (Fig. 1B, marked blue) have the ability to interact with polar-apolar lipid surfaces while the hydrophobic interface (Fig. 1B, yellow) may interact with the aliphatic chains of the lipids (Gimenez-Andres, Copic et al., 2018). Consistent with this lipid binding prediction, we found that the Atto488 labelled synthetic Apq12 peptide $(\mathrm{AaH})$ bound to uni-lamellar vesicles (GUVs; 1-10 $\mu \mathrm{M}$ in diameter; Fig. 1C). As control for the binding, we used the Atto488 dye alone, and introduced amino acid changes in the Atto488-labeled Apq12 peptide (AaH-ah) that abolished the helical hydrophobic moment, as an indication of the amphiphilicity of the helix (Eisenberg, Weiss et al., 1982), from 0.595 to 0.054 (Fig. 1B). The Atto488 dye and the AaH-ah peptide both failed to bind to liposomes (Fig. 1C).

139 To analyze the binding efficiency of AaH peptide in a more quantitative manner, SUV (small

140 uni-lamellar vesicles; 80-120 nm, Fig. S1A) bound peptide was separated from the unbound 141 peptide through a nycodenz gradient. Using SUVs composed of nuclear envelope (NE) and 142 plasma membrane (PM) lipids, we tested the impact of the lipid composition. NE derived 143 SUVs showed a higher AaH peptide binding efficiency compared to the PM SUVs (Fig. S1B). 
144 The Atto488 dye and the AaH-ah peptide did not bind to the SUVs in this experimental 145 regime (Fig. S1B). In addition, the peptide without liposomes was unable to float through the 146 nycodenz gradient (Fig. S1B, control).

148 Next, SUV binding of the AaH peptide was quantified using microscale thermophoresis 149 (MST) measurements. The $\mathrm{K}_{\mathrm{D}}$ of AaH peptide binding to NE-lipid derived SUVs was $16 \mu \mathrm{M}$ 150 (Fig. 1D). The binding affinity of the AaH peptide to $\mathrm{PM}$ derived SUVs was decreased to $K_{D}=$ $15159 \mu \mathrm{M}$ (Fig. 1D). Importantly, AaH-ah peptide failed to bind to any of these SUVs with a measurable $\mathrm{K}_{\mathrm{D}}$. Thus, the $\mathrm{AaH}$ peptide binds to liposomes depending on its amphipathic nature and the lipid composition of the liposomes.

The AaH of Apq12 localizes in the perinuclear space and is not required for subcellular localization and topology of Apq12

We analyzed whether the amphipathic nature of the $\mathrm{AaH}$ is important for the function of Apq12. Since apq12 $\Delta$ mutants show a growth defect at $16^{\circ} \mathrm{C}$ (Scarcelli et al., 2007), we tested APQ12, apq12 $\Delta$, apq12-ah and an additional AaH APQ12 mutant, apq12 F5DI6RV9N, for growth at different temperatures. The helical hydrophobic moment of the $\mathrm{AaH}$ was decreased in $\mathrm{AaH}^{\mathrm{FDDI6RV9N}}$ to 0.222 and therefore has an intermediate value between the WT $\mathrm{AaH}$ of Apq12 and AaH-ah of Apq12-ah (Fig. 1B). apq12-ah mutant completely failed to grow at $16^{\circ} \mathrm{C}$ and showed reduced growth at $23^{\circ} \mathrm{C}$, similar to the apq12 12 cells (Fig. $2 \mathrm{~A}$ ). In contrast, growth of apq12 55 DI6RV9N mutant cells was only reduced, but not completely abolished, at $16^{\circ} \mathrm{C}$ (Fig. 2A). Therefore, all further experiments were performed with apq12ah cells. Thus, the amphipathic nature of the AaH in Apq12 is important for cell growth at lower temperatures.

We next analyzed whether the subcellular localization of Apq12 requires the $\mathrm{A \alpha H}$. Consistent with published data on Apq12 distribution (Lone et al., 2015), yeGFP-Apq12 localized along the NE and the cell periphery, and a location that is probably the peripheral ER (Fig. 2B).

172 Similar localizations were observed for yeGFP-Apq12-ah (Fig. 2B). Thus, the integrity of the $\mathrm{AaH}$ of Apq12 is not important for the subcellular distribution of the protein.

Immuno-electron microscopy of yeGFP-Apq12 and yeGFP-Apq12-ah using GFP antibodies and protein A-gold detected both proteins along the INM, ONM, cytoplasmic and cortical ER (Fig. 2C and Fig. S1C). Apq12 was detected with the same frequency at the INM as at the ONM (Fig. S1C). For Apq12-ah there was a mild enrichment at the INM over the ONM (Fig. S1C). In addition, yeGFP-Apq12 and yeGFP-Apq12-ah associated with $10 \%$ and $16 \%$ of

180 NPCs, respectively, while most NPCs were not labelled (Fig. S1D). This may indicate a 
181 transient association of the proteins with assembling NPCs, as is the case for Brl1 and Brr6

182 (Zhang et al., 2018). In summary, Apq12 and Apq12-ah show similar subcellular 183 localizations.

Apq12 is a protein of the NE and the ER with two predicted membrane spanning regions (TM1 and TM2) that are connected by the AaH (Fig. 1A). To determine the topology of Apq12 and whether it requires a functional $\mathrm{AaH}$, we measured the accessibility of the $\mathrm{N}$ - and C-terminus of Apq12 and Apq12-ah by two approaches. First, we used the split GFP system for assessment of the localization of the $\mathrm{N}$ - and C-termini of Apq12 (Smoyer, Katta et al., 2016). The overlapping localization of $\mathrm{GFP}_{11}$ and $\mathrm{GFP}_{1-10}$ restores GFP fluorescence. Thus, by combining GFP $_{1-10}$ tagged versions of a protein with nuclear and cytoplasmic GFP $_{11}$ localised proteins, we can determine the topology of a protein. As a control, we used the perinuclear space localization of the C-terminus of the SUN-domain protein Mps3-GFP ${ }_{1-10}$ that failed to restore a fluorescent GFP signal when co-expressed with the ER and ONM localized GFP $_{11}$-mCherry-Scs2TM that carries GFP $_{11}$ at the ONM/ER on the cytoplasmic side and the nuclear GFP $_{11}$-mCherry Pus1 (Fig. 2D, top panel) consistent with published data (Smoyer et al., 2016). Interestingly, Apq12-GFP ${ }_{1-10}$ and GFP $_{1-10}-A_{p q 12}$ combined with GFP $_{11}$-mCherry Pus1 resulted in a green fluorescent NE signal and in case of GFP $_{11}$ mCherry-Scs2TM a NE/ER signal. These data show that the $\mathrm{N}$ - and C-termini of Apq12 are localized to either the cytoplasm or the nucleoplasm depending on whether Apq12 is at the ONM/ER or INM, respectively (Fig. 2D, middle). Very similar results were obtained for Apq12-ah (Fig. 2D, bottom).

As a further probe of Apq12 topology, we took advantage of the fact that biotin ligases are not contained in the perinuclear space, and assessed the ability of the $\mathrm{N}$ - or the C-termini of Apq12 and Apq12-ah to be biotinylated when fused with the histidine-biotin-histidine $(\mathrm{HBH})$ tag as a topology marker (Zhang et al., 2018). Pom152 that contains a short cytosolic Nterminal region, one TM domain and a C-terminal region in the perinuclear space was used as a control (Tcheperegine, Marelli et al., 1999). Consistent with the topology of Pom152, only the $\mathrm{N}$-terminal $\mathrm{HBH}$ tag but not the $\mathrm{C}$-terminal tag of Pom152 was biotinylated, demonstrating that the $\mathrm{HBH}$ biotinylation approach identifies the topology of NE proteins correctly (Fig. S1E). Both the $\mathrm{N}$ - and C-termini of Apq12 and Apq12-ah were biotinylated when fused to the $\mathrm{HBH}$ tag that carries a biotin acceptor site (Fig. S1E), confirming the findings from the split GFP approach. nucleoplasm, the $\mathrm{AaH}$ resides in the perinuclear space where it connects the two TM 
218 domains (Fig. 2E). A functional $\mathrm{A \alpha H}$ is not required for this topological arrangement of 219 Apq12. In order to understand how loss or impairment of the Apq12 function affects the NE and NPCs, we analyzed the phenotypes of $A P Q 12$, apq12 $\triangle$ and apq12-ah cells by electron microscopy (EM). As expected, APQ12 wild type cells had spherical nuclei with intact NE (Fig. 3A). Herniations as an indication of a defect in NPC biogenesis were detected as major phenotype in apq12 $\Delta$ (Fig. 3B and C, nucleus marked by red square) and apq12-ah mutants at $37^{\circ} \mathrm{C}$ (Fig. 3D and E, nucleus marked by red square). About $70 \%$ of the apq $12 \Delta$ mutants incubated at $23^{\circ} \mathrm{C}$ showed invaginations of the NE (Fig. $3 \mathrm{C}$ ). Herniations were relatively infrequent at this growth temperature. Invaginations of the NE and NE breakdown were the major defects of the apq12-ah mutant at $23^{\circ} \mathrm{C}$ (Fig. 3E). At $16^{\circ} \mathrm{C}$, the major defects of apq12 $\Delta$ cells were NE invaginations and NE breakdown followed by herniations (Fig. 3B and C). apq12-ah mutant showed NE breakdown and extrusions as major defects at 16ㅇ (Fig. 3D and E). Taken together, apq12 12 and apq12-ah mutants show phenotypic variations indicating that inactivation of the $\mathrm{AaH}$ does not cause the complete loss of Apq12 function. In addition, the consequence of Apq12 or AaH loss depends on the temperature. The rupture of the NE explains the lethality of apq12 $\Delta$ and apq12-ah cells at $16^{\circ} \mathrm{C}$. Loss of AaH function triggers a defect in NPC biogenesis at $37^{\circ} \mathrm{C}$.

It has been suggested that Apq12 has a function in lipid homeostasis (Lone et al., 2015), which might then account for the NE and NPC defects of apq12 and apq12-ah mutants. Therefore, we directly asked whether the AaH of Apq12 has an impact on lipid homeostasis, by incubating WT, apq12 1 and apq12-ah cells at $16^{\circ} \mathrm{C}, 30^{\circ} \mathrm{C}$ and $37^{\circ} \mathrm{C}$ and assessing their cellular lipid content by mass spectrometry. Overall apq12 $\Delta$ and apq12-ah mutants showed comparable lipid changes relative to WT (Fig. S2). The increase in ergosteryl ester (EE) and TAG and a decrease in ergosterol (Erg) and most species of glycerophospholipids (GPL) were prominent phenotypes in apq12 $\Delta$ and apq12-ah mutants (Fig. S2A and B), indicating a lipid metabolism flow from membrane lipids to storage lipids. In addition, we observed a significant decrease of double bonds in GPL in apq12 $\Delta$ and apq12-ah mutants (Fig. S2C). Moreover, the chain length (>34) in GPL was significantly increased in both APQ12 mutants (Fig. S2D). The reduction of membrane lipids, the decrease in the number of double bonds and the increase of chain length in GPL indicate a decrease in membrane fluidity in apq12 $\Delta$ and apq12-ah mutants, explaining the defects in NE breakdown at $16^{\circ} \mathrm{C}$ when the flexibility of membrane lipids will be reduced by the reduction in kinetic energy. These data suggests that the $\mathrm{AaH}$ of Apq12 does play a role in maintaining lipid homeostasis. 
Increased Apq12 levels are toxic to cells and cause the mis-localization of the NPC biogenesis factors Brl1 and Brr6 and the ER proteins Sec63 and Ole1

To gain deeper insights into the function of Apq12, we overexpressed $A P Q 12$ using the galactose-inducible $P_{\text {Gal1 }}$ promoter and followed the growth of these modified yeast cells. Because we lack an Apq12 antibody, we tagged Apq12 with 6His (Apq12-6His) to support immuno-detection of the fusion protein. Overexpression of $A P Q 12$ and APQ12-6His was equally toxic for cells (Fig. 4A). Such overexpression toxicity was not observed for the partner proteins $B R L 1$ and $B R R 6$ which also encode integral membrane proteins (Fig. 4B). Thus, of the three proteins in this functional module, only $A P Q 12$ has toxic consequences upon overproduction.

We next tested whether overexpression of $A P Q 12$ and $A P Q 12-6$ His impact on NE structure and function. We started by analyzing the impact of $A P Q 12$ over-expression on the distribution of the NPC biogenesis factors Brl1 and Brr6 (Fig. 4C-H; Fig. S3A and B). In vector control cells, yeGFP-Brl1 and yeGFP-Brr6 exhibited a uniform distribution throughout the NE (Fig. S3C and D). In contrast, one hour of induction of the $\mathrm{P}_{\text {Gal1 }}$ promoter to elevate Apq12 levels led to dense clustering of Brl1 and Brr6 on the NE (Fig. 4C-H, Fig. S3A and B). These clusters were devoid of the NPC marker Nup85-tdTomato (Fig. 4C, D, F and G, Fig. S3A and B). Clustering of yeGFP-Brl1 and yeGFP-Brr6 in response to APQ12-6His overexpression was also seen in time-lapse experiments (Fig. S3E and F). Because $\mathrm{P}_{\text {Gal1- }}$ $A P Q 12$ and $\mathrm{P}_{\text {Gal1 }}-A P Q 12-6 H$ is caused similar defects, we used $\mathrm{P}_{\text {Gal1 }}-A P Q 12-6$ His in further experiments.

We next asked how $A P Q 12-6 H i s$ overexpression affected the distribution of proteins at the NE/ER and INM, using the NE/ER proteins Sec63 and Ole1, the INM protein Asi3 and the ER luminal marker dsRED-HDEL as exemplars to test the impact upon NE/ER proteins (Feldheim, Rothblatt et al., 1992, Khmelinskii, Blaszczak et al., 2014). As seen for yeGFPBrl1 and yeGFP-Brr6, the smooth distribution of Sec63-yeGFP and Ole1-yeGFP along the $\mathrm{NE}$ was transformed into clusters as early as $1 \mathrm{~h}$ of $\mathrm{P}_{\mathrm{Gal} 1}-A P Q 12-6$ His induction (Fig. $4 \mathrm{C}, \mathrm{F}, \mathrm{I}$ and J). The localization of INM protein Asi3-yeGFP was initially unaffected by APQ12 overexpression, at the $1 \mathrm{~h}$ time point, however, by $3 \mathrm{~h}$ of $\mathrm{P}_{\text {Gal1 }}$ induction, modest clustering of Asi3-yeGFP along the NE emerged (Fig. S4A; white arrows). The ER marker dsRED-HDEL, that in vector control cells uniformly stained the NE and the cortical ER, started to accumulate in clusters within an hour of $\mathrm{P}_{\text {Gal1 }}-A P Q 12-6 H i$ is induction (Fig. S4B). Thus, increased Apq12 levels appears to impact upon the subcellular localization of ONM/ER proteins more strongly than the INM protein Asi3. 


\section{The luminal AaH of Apq12 contributes to toxicity}

293 We asked whether $\mathrm{AaH}$ is important for the deformation of the NE. PGal1-apq12-ah-6His

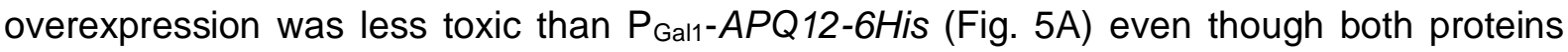
were overexpressed to similar levels (Fig. S5A). Consistent with the strongly reduced toxicity

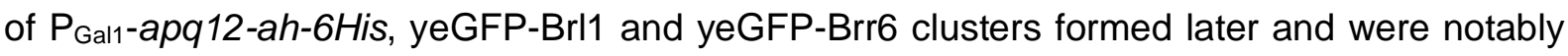

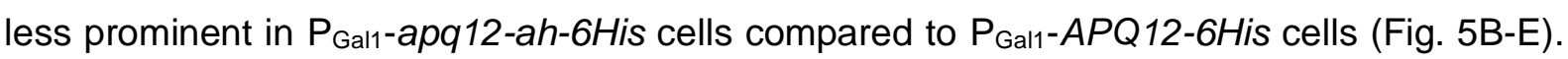
Thus, an intact $\mathrm{AaH}$ is a major factor in the toxic impact of $A P Q 12$ overexpression.

\section{Apq12 needs a functional AaH for the over-proliferation of the ONM and ER}

To understand how APQ12 overexpression leads to the changes on the ONM and ER, we

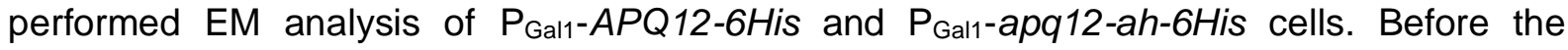
addition of galactose, the NE had uniform spherical morphology (Fig. S5B). Induction of $\mathrm{P}_{\mathrm{Gal1}}-\mathrm{APQ12}-6 \mathrm{His}$ led to a shift in the number of NE extensions (Fig. 6A-a, arrow) that were, in some cases, connected to the cortical ER (Fig. 6A-b) from 5\% to $40 \%$ (Fig. 6B). After 1 and $3 \mathrm{~h}$ of galactose addition, $\mathrm{P}_{\text {Gal1 }}-A P Q 12-6 H$ is cells contained ONM encircled vesicles containing granular material (Fig. 6A-c-f, red asterisks, and Fig. 6B). In contrast abnormal morphologies were not detected at the INM until $3 \mathrm{~h}$ after the induction of $A P Q 12$ overexpression, whereupon the INM formed small buds into the luminal space of the ONM vesicles (Fig. 6A-e, arrow). The consequences of apq12-ah-6His overexpression were less

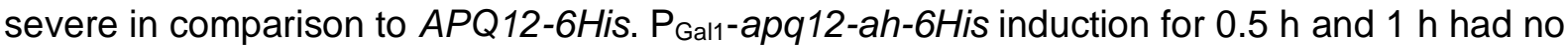
visible impact on the ONM or the ER in most cells (Fig. 6A-a'-d'). Only $3 \mathrm{~h}$ of $\mathrm{P}_{\text {Gal1-apq12-ah- }}$ 6 His induction caused proliferation of the ONM in $\sim 25 \%$ of the cell sections (Fig. 6A-e', arrows and Fig. 6B). This delayed accumulation of phenotypes in apq12-ah-6His cells is consistent with the reduced clustering of the BRL1-yeGFP and BRR6-yeGFP and impact on growth compared to APQ12-6His (Fig. 5).

Overexpression of $A P Q 12$ causes the accumulation of large, granular ONM encircled vesicles. To investigate the origin of the content in these vesicles, we expressed $\mathrm{P}_{\text {Gal1- }}$ APQ12-6His in cells with yeGFP tagged Brl1 as marker for the vesicles, and NLS-mRFP as nuclear marker. Overexpression of Apq12-6His induced yeGFP-Brl1 areas that were devoid of NLS-mRFP (Fig. 6C, top) indicating that these vesicles did not contain nuclear components. In a complementing experiment, we expressed $\mathrm{P}_{\text {Gal1 }}-A P Q 12-6$ His in RPL25yeGFP dsRed-HDEL cells with the green fluorescent ribosomal subunit as cytoplasmic marker and dsRed-HDEL as ONM/ER marker (Fig. 6C, bottom). Indeed, the dsRed-HDEL ONM extrusions co-localized with Rpl25-yeGFP, demonstrating that these vesicles contained cytoplasmic components. 
329 In a time-lapse experiment, we analyzed the cellular distribution of Apq12-yeGFP and 330 Apq12-ah-yeGFP upon $P_{\text {Gal1 }}$ expression (Fig. S5C). Apq12-yeGFP accumulated most 331 prominently at the NE extensions (Fig. S5C, 30 min, arrowheads). After 40 min of induction

332 Apq12-yeGFP localized in larger spots (Fig. S5C, arrowheads), consistent with the 333 accumulation of filled ONM deformations seen by EM (Fig. 6A). For the first 40 min of $P_{\text {Gal1 }}$ 334 induction Apq12-ah-yeGFP showed comparable localizations to Apq12-yeGFP (Fig. S5C). 335 However, due to its defective $\mathrm{AaH}$, Apq12-ah-yeGFP did not accumulate into larger spots. 336 Analysis of $\mathrm{P}_{\text {Gal1 }}$-APQ12-yeGFP after 0.5 and $3 \mathrm{~h}$ of galactose addition by immuno-EM 337 detected Apq12-yeGFP locating at NE extensions and ONM enriched vesicles (Fig. S5D). 338 Thus, the enrichment of Apq12 at ONM/ER sites induces membrane proliferation.

When taken together, $A P Q 12$ overexpression promotes extension of ER tubes from the ONM (Fig. 6D; steps 1 and 2). Fusion of these extensions with the NE (step 3) may entrap cytoplasmic content into ONM encircled compartments (step 4).

\section{APQ12 triggers PA accumulation at the NE dependent on its AaH}

The experiments above indicate that Apq12 promotes over-proliferation of the ONM and the $\mathrm{ER}$ in an $\mathrm{AaH}$ dependent manner. A mobilization of lipids by Apq12 would explain the ONM/ER expansion. To test for this possibility, we applied lipid mass spectrometry analysis

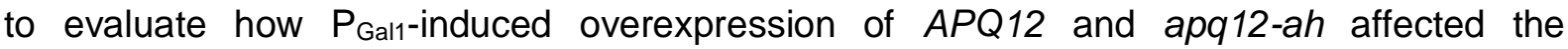
composition of cellular lipids. Cells carrying the pGal1 construct were used as vector control. Samples were analyzed at 0,1 and $3 \mathrm{~h}$ of galactose addition (Fig. S6A-D). Throughout the experiment the vector control behaved in a similar manner to apq12-ah cells. Interestingly,

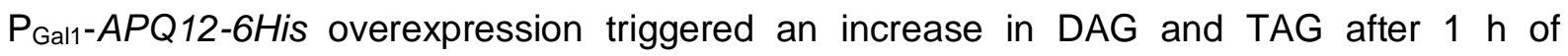

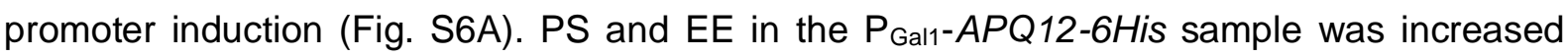
after $3 \mathrm{~h}$ induction. In addition, the number of two double bonds in glycerophospholipids (GPL) decreased in the $\mathrm{P}_{\text {Gal1 }}-A P Q 12-6$ His sample after $1 \mathrm{~h}$ but with an increase of one double bond after $3 \mathrm{~h}$. Furthermore, $\mathrm{P}_{\mathrm{Gal1}}-A P Q 12-6 H$ is expression also affected the chain length of GPL after $3 \mathrm{~h}$ of induction compared to $\mathrm{P}_{\mathrm{Gal1}}$-apq12-ah-6His induction (Fig. S6D). Thus, $\mathrm{P}_{\mathrm{Gal1}}-A P Q 12-6$ His expression has a mild impact on the lipid composition of cells.

Lipid mass spectrometry analysis did not enable us to make conclusions about local changes in lipid content, for example understanding whether the changes were restricted to one location such as the NE or cytoplasmic membrane systems. Using recently reported lipid sensors (Romanauska \& Kohler, 2018), we tested whether APQ12 overexpression affects PA accumulation at the INM or ONM and compared the outcome of overproduction of the wild type molecule with the impact of apq12-ah overexpression. We used the cytoplasmic 
366 Q2-mCherry and nuclear PA sensor NLS-Q2-mCherry for the analysis of PA changes at the 367 ONM and INM, respectively. Consistent with published data (Romanauska \& Kohler, 2018), at $\mathrm{t}=0$ strong Q2-mCherry decoration of the cytoplasmic membrane (Fig. 7A and B) was accompanied by a weak Q2-mCherry nuclear signal. After $1 \mathrm{~h}$ and $3 \mathrm{~h}$ of $\mathrm{P}_{\text {Gal1 }}-A P Q 12$ induction, the Q2-mCherry signal accumulated in a rim-like pattern at the NE and colocalized with developing yeGFP-Brl1 clusters at the NE (Fig. 7A-C). In $\mathrm{P}_{\text {Gal1-apq12-ah cells }}$ at $t=0$ the $Q 2-m C h e r r y$ reporter showed similar localization to $P_{\text {Gal1 }}-A P Q 12$ cells (Fig. 7A) and induction for $1 \mathrm{~h}$ did not impact the localization of the Q2-mCherry sensor. After $3 \mathrm{~h}$ of induction the nuclear Q2-mCherry signal was nearly completely diminished without directing the sensor to the NE (Fig. 7A-C).

Most interestingly, the nuclear NLS-Q2-mCherry $(\mathrm{t}=0)$ was recruited from a nuclear localization at $\mathrm{t}=0$ to a rim-like distribution at the NE 1-3 $\mathrm{h}$ after induction of $\mathrm{P}_{\text {Gal1 }}-A P Q 12$ 6His (Fig. 7D-F). In addition, NLS-Q2-mCherry strongly co-localized with yeGFP-Brl1 enrichments at the NE (Fig. 7D). In contrast, $\mathrm{P}_{\mathrm{Gal1}}$-apq12-ah expression did not impact the nuclear localization of NLS-Q2-mCherry (Fig. 7D-F). Thus, the AaH of APQ12 is required to induce PA enrichment at the NE. The co-localization of Apq12 and apq12-ah with a small subset of NPCs (Fig. S1D) raises the possibility that both proteins resemble Brl1 and Brr6 in associating with the newly assembled NPCs but not fully assembled NPCs (Zhang et al., 2018). We tested this notion by studying Apq12 and Apq12-ah localization in cells carrying temperature dependent $t d-b r l 1$ and td-brr6 degrons, that accumulate NPC biogenesis intermediates at $37^{\circ} \mathrm{C}$ (Zhang et al., 2018). Localization of Apq12 and Apq12-ah at herniations in nup116 cells was not analyzed because of the synthetically lethal phenotype of nup116 apq12 mutant combination (Scarcelli et al., 2007). Interestingly, in $t d-b r l 1$ and $t d-b r r 6$ cells incubated at $37^{\circ} \mathrm{C}$, yeGFP-Apq12 colocalized with Nup85-tdTomato while this was not the case in the equally treated WT control cells, as indicated by the scan of the NE signal (Fig. 8A and B). Quantification of the colocalization of yeGFP-Apq12 and Nup85-tdTomato signals along the NE showed a clear correlation between the Nup85-tdTomato and yeGFP-Apq12 signals in $t d$-brr6 and $t d$-brl1 cells, but not in WT control cells (Fig. $8 \mathrm{C}$ ). We next asked whether a functional AaH is required for the co-localization of Apq12 with NPC biogenesis intermediates. In a manner that is reminiscent of yeGFP-Apq12 behaviour (Fig. 8A and B), yeGFP-Apq12-ah co-

400 localized with Nup85-tdTomato in $t d$-brl1 and $t d$-brr6, but not WT cells (Fig. 8D and E). The 401 yeGFP-Apq12-ah and Nup85-tdTomato signals along the NE correlated in td-brr6 and td-br/1 402 cells but not in the WT control cells (Fig. 8F). This together suggests that Apq12 associates 
403 with NPC assembly intermediates in $t d-b r / 1$ and $t d-b r r 6$ cells and that the AaH of Apq12 is

404

405

406

407

408

409

410

411

412

413

414

415

416

417

418

419

420

421

422

423

424

425

426

427

428

429

430

431

432

433

434

435

436

437

438

439

not required for this localization.

In order to understand the precise localization of yeGFP-Apq12 and yeGFP-Apq12-ah in $t d$ brl1 and $t d$-brr6 cells in greater depth, immuno-EM analysis with anti-GFP antibodies was used to determine the subcellular distribution of these proteins. yeGFP-Apq12 was detected at, or inside, herniations (Fig. 8G and Fig. S7A). yeGFP-Apq12-ah was also recruited to herniations (Fig. 8E and Fig. S7A and B). To ensure that the Apq12 enrichment was not an indirect consequence of the increase in membrane surface in herniations compared to the $\mathrm{NE}$, we measured gold particle number relative to the length of the sectioned membrane along NE and in herniations. This analysis detected less than 1 gold particle per $\mu \mathrm{m}$ of the NE in WT, $t d$-brl1 and $t d$-brr6 cells (Fig. S7C). In herniations, this number was increased to 5 gold particles per $\mu \mathrm{m}$ clearly indicating an enrichment of Apq12 with herniations.

\section{Interaction of Brl1 and Brr6 is regulated by AaH of APQ12}

Apq12 shows physical and genetic interactions with BRL1 and BRR6 (Hodge et al., 2010, Lone et al., 2015, Scarcelli et al., 2007). A dysfunctional AaH could have an impact on Brl1 and Brr6 behaviour and their interaction with Apq12. Analysis of the Brl1 protein indicated an increase of Brl1 levels in apq12 1 and apq12-ah mutants (Fig. 9A and B) compared to WT. This increase was more pronounced in apq12 $\Delta$ mutants (Fig. 9A and B). Because of the lack of Brr6 antibodies, we tested the abundance of Brr6-yeGFP in WT, apq12 $\Delta$ and apq12-ah mutants. apq12 $\Delta$ showed lethality in combination with BRR6-yeGFP and was therefore not tested. The Brr6-yeGFP level was increased in apq12-ah mutant compared to the WT (Fig. $9 \mathrm{C}$ and D). Analysis of the localization of Brl1-yeGFP and Brr6-yeGFP in apq12-ah mutants showed cellular distributions of both proteins that were reminiscent of that seen in WT (Fig. $9 \mathrm{E})$, with an increase in signal intensity along the NE and cortical ER in apq12-ah mutants (Fig. 9E, Grey Value of the NE scans). This is consistent with the increase in protein levels of Brl1 and Brr6 in apq12-ah cells (Fig. 9A-D).

We next analyzed whether the increase in protein levels of Brl1 and Brr6 in mutants is also reflected in Brl1-Brr6 interaction in co-IP experiments in which Brr6-yeGFP was immunoprecipited with anti-GFP antibodies followed by the analysis of the immunoprecipitated proteins with Brl1 and Apq12-6HA antibodies. The Brr6-yeGFP immunoprecipitation showed complex formation of Apq12 and Apq12-ah with Brl1 and Brr6 (Fig. 9F). Quantification of the immunoprecipitated proteins from three independent experiments showed that although Brr6-yeGFP precipitation efficiency was very similar, more Apq12 and Brl1 were co-immunoprecipitated from apq12-ah cells than from APQ12 cells 
440 (Fig. 9F and G). Thus, a defect in AaH of Apq12 enhances the interaction between Brr6, Brl1 441 and Apq12.

\section{Discussion}

444 Apq12 is important for NPC function and cooperates with the essential NPC biogenesis 445 factors Brl1 and Brr6 (Hodge et al., 2010, Lone et al., 2015, Scarcelli et al., 2007). These 446 data indicate that Apq12 is at the heart of machinery that ensures NPC assembly, raising the 447 important question about its molecular function. Because Apq12 does not have any 448 sequence similarities to proteins with enzymatic activity, we have to assume that it functions 449 either as a protein scaffold or impacts the shape of the NE as reported for reticulons that 450 bend the ER by two cooperating pairs of trans-membrane domains with an adjacent $\mathrm{AaH}$ 451 (Hu, Shibata et al., 2008, Wang, Clark et al., 2021). Following the rational that a combination 452 of transmembrane domains and $\mathrm{AaH}$ could be the basis for the functional of Apq12, we 453 analyzed Apq12 by the AmphipaSeeK program that predicated the presence of a short $454 \mathrm{AaH}$ connecting the two transmembrane domains. Consistent with this predication, the 455 synthetic $\mathrm{AaH}$ peptide binds to liposomes dependent on its amphipathic nature and the lipid composition. In addition, topological analysis showed that the $\mathrm{AaH}$ resides in the intermembrane space of the NE, while $\mathrm{N}$ - and C-terminal regions of Apq12 localize in the nucleus or cytoplasm depending on the INM or ONM localization of Apq12.

In terms of cold sensitivity, NE and NPC biogenesis defects the apq12-ah mutant behaves similar to complete loss of APQ12 function (this study, (Hodge et al., 2010, Lone et al., 2015, Scarcelli et al., 2007). However, phenotypic differences between apq12-ah and apq12 $\Delta$ are also detectable (Fig. 3). In addition, Apq12-ah associates at least as efficiently with Brl1 and Brr6 as Apq12, has the same topological arrangement as Apq12 and associates with NPC assembly intermediates. This together indicates that apq12-ah is not a loss of function allele. The $\mathrm{AaH}$ is required for the overall function of Apq12. However, $\mathrm{N}$ - and C-termini of Apq12 fulfill functions without the involvement of $\mathrm{AaH}$. This probably explains why prolonged overexpression of apq12-ah still shows a mild membrane deforming phenotype (Fig. 5). apq12-ah and apq12 $\Delta$ mutants impact cellular lipid composition, independent of the tested growth temperature, in a way that reduces membrane fluidity. This accounts for the cold sensitive growth defect and the NE breakdown phenotype at reduced growth temperatures. In addition, we observed the striking accumulation of PA either through synthesis or relocalization at the NE upon overexpression of Apq12, dependent on the functionality of the 
476

et al., 2021) raising the question as to whether PA accumulation at the $N E$ induced by $P_{\text {Gal1- }}$ APQ12 overexpression is a consequence of defective NPCs. The observation that $\mathrm{P}_{\text {Gal1- }}$ $A P Q 12$ overexpression merely displaces NPCs into areas that lack ONM expansions without the accumulation of defective NPC assemblies (Figs. 4C and S3A), indicates that overproduced Apq12 has the ability to induce PA accumulation at the NE even when NPCs are intact.

Before we have shown that INM membrane deformations in $t d$-brl1 and $t d$-brr6 cells reflect emerging NPCs that do not fully assemble because of a defect in one of the steps leading to the generation of functional NPCs (Zhang et al., 2018). Using cells carrying $t d-b r / 1$ and $t d$ brr6 we observed strong accumulation of Apq12 with NPC biogenesis intermediates and this localization does not require a functional $\mathrm{A \alpha H}$. In contrast, Apq12 only co-localizes with a small number of NPCs in WT cells. Thus, Apq12 joins Brl1 and Brr6 (Zhang et al., 2018) in a small subset of proteins required for NPC assembly that transiently interact with assembling NPCs but then dissociate from NPCs as soon as they are fully assembled.

As Apq12, Brl1 and Brr6, the Lap2-emerin-MAN1 (LEM) family proteins Heh1 and Heh2 are also nonstructural components of NPCs. However, in contrast to Apq12, Brl1 and Brr6 that function directly in NPC biogenesis, Heh1 and Heh2 contribute to the surveillance of NPC biogenesis (Webster, Colombi et al., 2014). Upon NE damage caused by a NPC biogenesis defect, Chm7, a component of an ESCRT-III like complex, enters the nucleus where it is activated by nuclear Heh1 to promote the sealing of the disrupted NE by the ESCRT machinery (Thaller et al., 2019, Webster et al., 2014). In contrast to Heh1, Heh2 probably functions as a senor for the assembly state of NPCs (Borah, Thaller et al., 2021). Thus, Apq12, Brl1 and Brr6 and Heh1 and Heh2 fulfill quite distinct functions at NPCs.

Based on our findings and the observation that the role of the Apq12-Brl1/Brr6 module overlaps with that of the nucleoporin Nup116 in scaffolding NPC biogenesis (Onischenko et al., 2017, Zhang et al., 2018), we suggest the following stages for the stepwise assembly of NPC assembly. The FG nucleoporin Nup116 was shown to scaffold NPC assembly together with Nup188 on the nuclear side of the INM (Onischenko, Noor et al., 2020, Onischenko et al., 2017). As Nup116, Apq12, Brl1 and Brr6 also associate with NPC intermediates (van Leeuwen, Pons et al., 2020, Zhang et al., 2018 and this study). The interaction of Brl1 with the integral membrane protein Ndc1 (Winey, Hoyt et al., 1993) and Nup188 could be the trigger for the recruitment of this protein to assembling NPCs (Zhang et al., 2018). How Apq12 is recruited to NPC intermediates is not understood. The interacting Brl1 and Brr6 are 
512 not essential for this localization since Apq12 associates with herniations upon induced 513 depletion of Brl1 and Brr6 (Fig. 8).

Apq12 probably executes multiple functions, some of which are at least partly mediated by its $516 \mathrm{AaH}$ at NPC biogenesis sites. First, Apq12 inserts through its membrane active AaH into the 517 NE from within the intermembrane space (Fig. 1). This insertion has the potential to generate 518 membrane curvature that may stabilize membrane deformations that arise during NPC 519 biogenesis. AaH peptide did not deform GUVs under the experimental conditions we applied 520 (Fig. 1). However, the yeast reticulon Yop1 only deforms liposomes in the context of transmembrane domains and $\mathrm{A \alpha H}$ (Wang et al., 2021). Thus, in future experiments it will be important to measure the membrane-deforming ability of the TM-AaH-TM core of Apq12. Second, the enrichment of Apq12 at NPC biogenesis sites may promote local accumulation of PA as indicated by the $A P Q 12$ overexpression phenotype. Because of its conical shape, PA could stabilize bent membrane regions at INM bends during NPC biogenesis (Zhukovsky, Filograna et al., 2019). In addition, PA has the ability to interact with a range of proteins via short stretches of positively charged amino acid residues (Jang, Lee et al., 2012, Tanguy, Kassas et al., 2018). Presently, no Nup or NPC biogenesis factor with PA binding activity has been described. However, it was only until recently that the PA-binding activity of the ESCRTIII protein Chm7 was reported (Thaller et al., 2021), raising the possibility that proteins involved in NPC assembly may carry hidden PA binding sites. Third, considering that Apq12 has an impact on Brl1 and Brr6 levels and the interaction of Apq12, Brl1 and Brr6 (Fig. 9), it may coordinate localization of the interacting Brl1 and Brr6 at NPCs biogenesis sites, a function that would be most important at elevated temperatures when Apq12 most strongly plays a role in NPC assembly. APQ12, BRL1 and NUP116 show genetic interactions and overexpression of $B R L 1$ is able to suppress the NPC biogenesis defect of nup116 $\triangle$ and

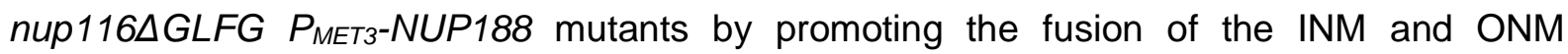
(Onischenko et al., 2017, Scarcelli et al., 2007, Zhang et al., 2018). We therefore suggest that Brl1, in part scaffolded by Apq12, directly or indirectly, facilitates the fusion between the INM with the ONM during NPC biogenesis.

542 The Apq12-Brl1/Brr6 module is only conserved in organisms with closed mitosis (Tamm et 543 al., 2011). However, considering the simple domain architecture of Apq12 with two TM 544 domains and a short $\mathrm{AaH}$, proteins with similar organisation may substitute for the function of 545 Apq12 in higher eukaryotes without having detectable amino acid homology to Apq12, rather 546 a structural similarity with two closely opposed TM domains. Brl1 and Brr6 have an extended 547 intermembrane space domain that is stabilized by two functionally important disulphide 548 bridges (Tamm et al., 2011, Zhang et al., 2018). This stabilization principal could be 
549 substituted for alternative structural features in functional equivalent proteins in higher 550 eukaryotes.

\section{Materials and Methods}

\section{Yeast strains and plasmids}

555 Yeast strains and plasmids used in this study are listed in Table S1. All yeast strains are derived from ESM356-1 (MATa ura3-52 trp1 463 his3 $\Delta 200$ leu2 $\Delta$ 1). Gene deletion and epitope tagging of endogenous genes was performed using a PCR-based integration approach (Janke, Magiera et al., 2004, Knop, Siegers et al., 1999). Yeast strains were grown in SC (synthetic complete) medium, SC-selection medium (Guthrie \& Fink, 1991), YPD (yeast extract, peptone and glucose) or YPRaf (yeast extract, peptone and raffinose) with or without $0.1 \mathrm{mM} \mathrm{CuSO}_{4}$ at $23^{\circ} \mathrm{C}, 30^{\circ} \mathrm{C}$ or $37^{\circ} \mathrm{C}$. Galactose was added to a final concentration of $2 \%$ to induce expression of genes under control of the $P_{\text {Gall }}$ promoter. Alkaline lysis and TCA precipitations were used to prepare yeast extracts in order to analyse protein levels by immunoblotting (Janke et al., 2004). To test for growth defects, yeast cells were grown over night in the indicated selection medium, afterwards the cell density was adjusted to $\mathrm{OD}_{600}=$ 1. The cell suspension was then spotted in 10 -fold serial dilutions onto selection plates that were incubated as indicated.

\section{EM and immuno-EM analysis of yeast cells}

570 Cells were high pressure frozen, freeze-substituted, sectioned, labelled and stained for electron microscopy as described (Giddings, O'Toole et al., 2001). Briefly, cells were collected onto a $0.45 \mu \mathrm{m}$ polycarbonate filter (Millipore) using vacuum filtration and then high pressure frozen with a HPM010 (Abra-Fluid, Switzerland). Cells were freeze-substituted using the EM-AFS2 device (Leica Microsystems, Vienna, Austria) (freeze substitution solution: $0.1 \%$ glutaraldehyde, $0.2 \%$ uranyl acetate, $1 \%$ water - dissolved in anhydrous acetone) and stepwise infiltrated with Lowicryl HM20 (Polysciences, Inc., Warrington, PA), started at $-90^{\circ} \mathrm{C}$. For polymerization the samples were exposed to UV light for $48 \mathrm{~h}$ at $-45^{\circ} \mathrm{C}$ and gradually warmed up to $20^{\circ} \mathrm{C}$. Embedded cells were serially sectioned using a Reichert Ultracut S Microtome (Leica Instruments, Vienna, Austria) to a thickness of $80 \mathrm{~nm}$. Poststaining with $3 \%$ uranyl acetate and lead citrate was performed. Sections were imaged at a Jeol JE-1400 (Jeol Ltd., Tokyo, Japan) operating at $80 \mathrm{kV}$ equipped with a 4k $x$ 4k digital camera (F416, TVIPS, Gauting, Germany). Micrographs were adjusted in brightness and contrast using ImageJ. For immuno-labelling the primary antibody was used against GFP. 
585 from the freeze substitution solution. The sections were treated with blocking buffer (1.5\% 586 BSA, $0.1 \%$ fish skin gelatin in PBS), then incubated with the primary antibody, followed by 587 treatment with protein A-gold conjugates $(10 \mathrm{~nm}$, Utrecht University, Utrecht, The 588 Netherlands).

589

590 Fluorescence light microscopy and image analysis

591 A DeltaVision RT system (Applied precision, Olympus IX71 based) equipped with the 592 Photometrics CoolSnap HQ camera (Roper Scientific), a 100x/1.4 NA UPlanSAPO objective 593 (Olympus), a mercury arc light source and the softWoRx software (Applied Precision) was 594 used for cell imaging. Imaging was done at $16^{\circ} \mathrm{C}, 23^{\circ} \mathrm{C}, 30^{\circ} \mathrm{C}$ or $37^{\circ} \mathrm{C}$ using the GFP, and 595 the mCherry channels with different exposure times according to the fluorescence intensity of 596 each protein. For time-lapse experiments, cells were immobilized on Concanavalin A (Sigma597 Aldrich)-coated 35-mm glass bottomed dishes (P35G-1.5-14C; MatTek Corporation) and 598 kept in their respective media. Images were deconvolved with the softWoRx software 599 (Applied Precision) and processed with ImageJ (National Institutes of Health, Bethesda MD). 600 For Fig. 5 C and E, Quantification of maximum intensity of individual cells was done by 601 utilising CellProfiler 3.1.9 software (Broad Institute, Cambridge, MA) (Carpenter, Jones et al., 602 2006).

603

\section{Lipid analysis by mass spectrometry}

$60510 \mathrm{OD}$ of cells were harvested and homogenized using a FastPrep machine (MP 606 Biomedicals) in $155 \mathrm{mM}$ ammonium bicarbonate buffer at $\mathrm{pH}$ 7.5. Homogenized cells were 607 subjected to acidic Bligh\&Dyer lipid extractions in the presence of internal lipid standards 608 added from a master mix containing PC (phosphatidylcholine, 13:0/13:0, 14:0/14:0, 609 20:0/20:0; 21:0/21:0, Avanti Polar Lipids), PI (phosphatidylinositol, 17:0/20:4, Avanti Polar 610 Lipids), PE and PS (phosphatidylethanolamine and phosphatidylserine, 14:1/14:1, 20:1/20:1, 611 22:1/22:1, semi-synthesized as described (Ozbalci, Boyaci et al., 2013), DAG (diacylglycerol, 612 17:0/17:0, Larodan), TAG (triacylglycerol, D5-TAG-Mix, LM-6000/D5-TAG 17:0,17:1,17:1, 613 Avanti Polar Lipids), PA (phosphatidic acid, 17:0/20:4, Avanti Polar Lipids), PG 614 (phosphatidylglycerol, 14:1/14:1, 20:1/20:1, 22:1/22:1, semi-synthesized (Ozbalci et al., 615 2013), and Cer (ceramide, Avanti Polar Lipids). Lipids recovered in the organic extraction 616 phase were evaporated by a gentle stream of nitrogen. Prior to measurements, lipid extracts 617 were dissolved in $10 \mathrm{mM}$ ammonium acetate in methanol and transferred to 96 -well plates 618 (Eppendorf twintec plate 96). Mass spectrometric measurements were performed in positive 619 ion mode on an AB SCIEX QTRAP 6500+ mass spectrometer, equipped with chip-based 620 (HD-D ESI Chip, Advion Biosciences) nano-electrospray infusion and ionization (Triversa 621 Nanomate, Advion Biosciences) as described (Ozbalci et al., 2013). The following precursor 
622 ion scanning (PREC) and neutral loss scanning (NL) modes were used for the measurement

623 of the various lipid classes: +PREC 184 (PC), +PREC282 (t-Cer), +NL141 (PE), +NL185 624 (PS), +NL277 (PI), + NL189 (PG), +NL115 (PA), +PREC 77 (ergosterol), + PREC379 625 (ergosteryl ester). Ergosterol was quantified following derivatization to ergosterol acetate in 626 the presence of the internal standard (22E)-Stigmasta-5,7,22-trien-3-beta-ol (Aldrich, 627 R202967) using $100 \mu \mathrm{l}$ acetic anhydride/chloroform (1:12 v/v) (Ejsing, Sampaio et al., 2009). 628 Data evaluation was done using LipidView (ABSciex) and an in house-developed software 629 (ShinyLipids).

630

\section{Liposome preparation}

632 All lipids were received from Avanti Polar lipids with the exception of Atto647N, which was 633 obtained from Atto-Tec. The lipid composition of the PM mix consisted of: $34.8 \mathrm{~mol} \%$ 634 1-palmitoyl-2-oleoyl-sn-glycero-3-phosphocholine (POPC), 15 mol\% 1,2-dioleoyl-sn-glycero635 3-phosphoserine (DOPS), 20 mol\% 1-hexadecanoyl-2-octadecenoyl-sn-glycero-3-phospho636 ethanolamine (POPE), $25 \mathrm{~mol} \%$ cholesterol (from ovine wool), $5 \mathrm{~mol} \%$ liver L- $\alpha-$ 637 phosphatidylinositol (PI, from liver) and $0.2 \mathrm{~mol} \%$ Atto647N-DPPE. The composition of the 638 used nuclear envelope (NE) lipid mix was: 19.8 mol\% POPC, 3 mol\% DOPS, 42 mol \% 639 Cholesterol, 7 mol\% POPE, $23 \mathrm{~mol} \% \mathrm{PI}, 5 \mathrm{~mol} \% \mathrm{PI}(4,5) \mathrm{P}_{2}$, and $0.2 \mathrm{~mol} \%$ Atto647 (Zhendre, 640 Grelard et al., 2011). SUVs (small uni-lamellar vesicles) were formed as described previous 641 (Weber, Zemelman et al., 1998) by dissolving the lipid mixtures in Octyl- $\beta$-D glucopyranoside 642 (OG) containing buffer, OG dilution below the critical micellar concentration, flow dialysis and 643 SUV isolation using Nycodenz-gradient centrifugation. Subsequently, the concentrated 644 liposomes were extruded 23-times with a $100 \mathrm{~nm}$ filter and stored at $4^{\circ} \mathrm{C}$.

645 For preparation of GUVs (giant uni-lamellar vesicles) the PM mix was used. SUVs were 646 prepared to produce GUVs as described previously (Malsam, Parisotto et al., 2012) with the 647 following modifications: (I) the GUVs were desalted two times employing a PD10 column (GE 648 Healthcare) instead of using a Sephadex-G50 gel filtration column in the second desalting 649 step. (II) platinum-coated glass slides (GeSiM) were applied instead of ITO-coated glass 650 slides (GeSiM) (Meijer, Dorr et al., 2018).

651 The lipid concentration was measured by the fluorescence of Atto647N with an excitation of $652647 \mathrm{~nm}$ and an emission of $670 \mathrm{~nm}$ using a Fluoroskan Ascent FL plate reader (Thermo 653 Scientific) in a 96-well plate (integration time: $1000 \mathrm{~ms}$ ). Therefore, the liposomes were 654 disrupted by $0.5 \%$ Dodecyl-maltoside (DDM). The recovery of the total lipid was compared to 655 the lipid after preparation. 
660 DLS was applied to monitor the size of the liposomes. After adding $2 \mu \mathrm{M}$ lipid (5 $\mu$ final 661 volume) to the quartz cuvette, the particle size was determined in a DynaPor NanoStar 662 (Wyatt technologies) instrument at room temperature. The buffer composition for size 663 determination was set to PBS and 20 acquisitions with $5 \mathrm{~s}$ time intervals were measured.

664

\section{Binding studies of SUVs}

666 The binding of the $\mathrm{AaH}$ and $\mathrm{AaH}$-ah peptides (from PSL Peptide Specialty Laboratories $667 \mathrm{GmbH}$, Heidelberg; amino acid sequence: KLLMNFITLVKRFL, KLDMNRNTLVKRNL) 668 coupled with Atto488 (freshly solved in DMSO and diluted in $25 \mathrm{mM} \mathrm{HEPES} \mathrm{pH} \mathrm{7.4,} 135 \mathrm{mM}$ $669 \mathrm{KCl}, 1 \mathrm{mM}$ DTT (fusion buffer)) with SUVs was measured by a Nycodenz gradient 670 centrifugation. $0.45 \mathrm{mM}$ lipid was incubated with $18 \mu \mathrm{M} \mathrm{AaH}$ or $\mathrm{AaH}$-ah peptide or $18 \mu \mathrm{M}$ 671 Atto488 in $25 \mathrm{mM}$ HEPES pH7.4, $135 \mathrm{mM} \mathrm{KCl}, 2.1 \%$ DMSO and $1 \mathrm{mM}$ DTT for 30 minutes 672 at room temperature, whereas $10 \%$ of the sample was used as the reference input. The 673 peptide without liposomes functioned as a floatation control. Nycodenz (solved in fusion 674 buffer) was added to the sample producing a $40 \%$ Nycodenz layer in the bottom of the centrifuged tube. The sample was overlaid with decreasing concentrations of Nycodenz (30\%, 20\%, 10\% and 0\%) and centrifuged in a SW55 rotor (Beckman Coulter) 2h 30 min at $55000 \mathrm{rpm}$ and $4^{\circ} \mathrm{C}$. The floated SUVs were located in the layer between $10 \%-20 \%$ Nycodenz. The recovery of the SUVs was measured by the fluorescence of Atto647N in the DDM lysed sample and compared to the input. The binding of the peptide or Atto488 was analyzed by the fluorescence signal of Atto488 (excitation: $460 \mathrm{~nm}$, emission: $538 \mathrm{~nm}$, integration time: $1000 \mathrm{~ms}$ ) in this layer and compared to the input probe. The binding of the peptide was corrected to the amount of the floated SUVs.

\section{Binding studies of GUVs}

685 For the peptide interaction to GUVs, 3.3 $\mu \mathrm{M}$ GUVs were incubated with different amounts of peptide in a ratio of $1: 1$ to $10: 1$ in fusion buffer at room temperature for 15 minutes. As a control analysing the change of the GUV morphology $0.04 \%$ DMSO was added to the GUVs without peptide. The morphology of the GUVs was visible in the fluorescence of Atto647 and the interaction of the peptide by the fluorescent signal of Atto488. It was measured in a chambered coverslip (Ibidi, catalogue No. 80826) using a DeltaVision microscope.

693 For determination of the $\mathrm{K}_{\mathrm{D}}$ values via MST $2 \mu \mathrm{M}$ Atto488 coupled to $\mathrm{AaH}$ and $\mathrm{AaH}$-ah 694 peptide or Atto488 as a control was incubated 15 minutes at room temperature with 695 increasing liposome concentrations in fusion buffer with $0.01 \%$ Triton $X-100$. All samples 
696 were filled in Premium capillaries and the thermophoresis was measured in a Monolith

697 NT.115 (NanoTemper) instrument with a LED power of 40\% (green filter) and an MST power 698 of $17 \%$. The MST data were evaluated using a single exponential function in consideration of 699 the initial state calculated by linear regression. The data were normalized to the ratio of 700 bound/unbound peptide and the $K_{D}$ value was determined according to the Hill equation:

$701 \quad y=\frac{[c]^{n}}{[c]^{n}+K_{D}{ }^{n}}$ whereas $\mathrm{n}=$ Hill slope.

702

703

704

705

706

707

708

709

710

711

712

713

714

715

716

717

718

719

720

721

722

723

724

725

726

\section{Immunoprecipitation}

Cells (25 OD) were harvested and resuspended in lysis buffer $(20 \mathrm{mM}$ Tris- $\mathrm{Cl}, \mathrm{pH}$ 8.0, $150 \mathrm{mM} \mathrm{NaCl}, 5 \mathrm{mM} \mathrm{MgCl}_{2}$, and $10 \%$ glycerol) supplemented with $10 \mathrm{mM} \mathrm{NaF}$, $60 \mathrm{mM}$-glycerophosphate, 1 tablet/50 $\mathrm{ml}$ Roche protease inhibitor cocktail complete (EDTA free), and $1 \mathrm{mM}$ PMSF. Glass beads (BioSpec Products) were added, and cells were lysed in a FastPrep machine (MP Biomedicals). Cell lysate was supplemented with $0.5 \%$ Triton X-100 and incubated on ice for $10 \mathrm{~min}$. The soluble proteins were separated from the cell debris by centrifugation and incubated with GFP-Trap agarose beads (Chromotek) at $4^{\circ} \mathrm{C}$ for $2 \mathrm{~h}$. Beads were washed three times with lysis buffer supplemented with $0.1 \%$ Triton X-100 and twice with wash buffer ( $20 \mathrm{mM}$ Tris- $\mathrm{Cl}, \mathrm{pH} 8.0,150 \mathrm{mM} \mathrm{NaCl}$, and $5 \mathrm{mM} \mathrm{MgCl}$ ). Bound proteins were eluted in $50 \mu \mathrm{l}$ of $2 \times$ SDS-PAGE sample buffer, heated to $95^{\circ} \mathrm{C}$ for $5 \mathrm{~min}$, separated by SDS-PAGE, and transferred to PVDF membrane (Millipore) for Western blotting.

\section{Statistical analysis}

For the statistical analyses, PRISM v.7 software (GraphPad) was used. Comparisons of samples were performed using unpaired $t$ test with two-tailed $\mathrm{p}$-value. Data distribution was assumed to be normal, but this was not formally tested.

\section{Antibodies}

Antibodies and their conditions of use are as follows: mouse anti-His (Western blot, 1:1000; 34660; Qiagen), rabbit anti-Brl1 (Western blot, 1:1000; made in-house), rabbit anti-Tub2 (Western blot, 1:1000; made in-house), and rabbit anti-GFP (Western blot, 1:1000; Proteintech), rabbit anti-HA (Western blot, 1:500; Proteintech), rabbit anti-GFP (immuno-EM, 1:5; gift from M. Seedorf, Zentrum für Molekulare Biologie, Heidelberg, Germany). 


\section{Acknowledgements}

728 We thank the electron microscopy facility of Heidelberg University for their support. This work

729 is supported by a grant of the Deutschen Forschungsgemeinschaft (DFG) to E. Schiebel 730 (DFG Schi 295/5-3) and to B. Brügger (Project Number 112927078 - TRR 83). We thank A.

731 Köhler for the gift of the PA-mCherry sensor plasmids and lain Hagan for the Brl1 antibody.

732

\section{Online supplementary material}

734 Fig. S1 shows the quantification of localization data from immuno-EM. It also includes data 735 showing the topology of Apq12 and apq12-ah by biotinylation of the $\mathrm{HBH}$ tag. Fig. S2 shows 736 the lipid analysis of WT, apq12 $\triangle$ and apq12-ah cells. Fig. S3 shows time courses and time 737 lapses of Apq12 overexpression under the galactose promoter in yeGFP-BRL1 NUP85738 tdTomato and yeGFP-BRR6 NUP85-tdTomato cells. Fig. S4 shows effects of APQ12 739 overexpression on the INM protein Asi3 and ER luminal marker HDEL. Fig. S5 shows the 740 localization of overexpressed Apq12-yeGFP and apq12-ah-yeGFP with cellular sites of 741 membrane over proliferation. Fig. S6 shows the lipid analysis of cells with overexpression of 742 Apq12 and Apq12-ah. Fig. S7 shows immuno-EM images of yeGFP-Apq12 and apq12-ah743 yeGFP localizing with herniations.

\section{Author Contributions}

\section{Wanlu Zhang}

747 Zentrum für Molekulare Biologie der Universität Heidelberg, DKFZ-ZMBH Allianz,

748 Heidelberg, Germany.

749 Cell Biology and Biophysics Unit, European Molecular Biology Laboratory (EMBL),

750 Heidelberg, Germany.

751 Contribution: Conceptualization, Investigation, Formal analysis, Visualization, Review and 752 editing

753 Competing Interest: No competing interests declared

754

\section{Azqa Khan}

756 Zentrum für Molekulare Biologie der Universität Heidelberg, DKFZ-ZMBH Allianz, 757 Heidelberg, Germany.

758 Contribution: Investigation, Formal analysis, Data curation and visualization, Review and 759 editing

760 Competing Interest: No competing interests declared 


\section{Jlenia Vitale}

764 Zentrum für Molekulare Biologie der Universität Heidelberg, DKFZ-ZMBH Allianz,

765 Heidelberg, Germany.

766 Contribution: Investigation, Formal analysis and visualization

767 Competing Interest: No competing interests declared

Annett Neuner

770

Zentrum für Molekulare Biologie der Universität Heidelberg, DKFZ-ZMBH Allianz,

771 Heidelberg, Germany.

772 Contribution: EM and immune-EM and analysis

773 Competing Interest: No competing interests declared

\section{Kerstin Rink}

776 Biochemie-Zentrum der Universität Heidelberg, Heidelberg, Germany

777 Contribution: Design and analysis of lipid binding experiments

778 Competing Interest: No competing interests declared

\section{Christian Lüchtenborg}

781 Biochemie-Zentrum der Universität Heidelberg, Heidelberg, Germany

782 Contribution: Lipid mass spectrometry analysis

783 Competing Interest: No competing interests declared

\section{Britta Brügger}

786 Biochemie-Zentrum der Universität Heidelberg, Heidelberg, Germany

787 Contribution: Lipid mass spectrometry analysis

788 Competing Interest: No competing interests declared

\section{Thomas H. Söllner}

791 Biochemie-Zentrum der Universität Heidelberg, Heidelberg, Germany

792 Contribution: Design and analysis of lipid binding experiments

793 Competing Interest: No competing interests declared

\section{Elmar Schiebel}

796 Zentrum für Molekulare Biologie der Universität Heidelberg, DKFZ-ZMBH Allianz,

797 Heidelberg, Germany.

798 Contribution: Conceptualization, Supervision, Funding acquisition, Writing, Review and editing 
800 For Correspondence: e.schiebel@zmbh.uni-heidelberg.de

801 Competing Interest: No competing interests declared

802

\section{References}

804 Aitchison JD, Blobel G, Rout MP (1995) Nup120p: A yeast nucleoporin required for

805 NPC distribution and mRNA transport. Journal of Cell Biology 131: 1659-1675

806 Allegretti M, Zimmerli CE, Rantos V, Wilfling F, Ronchi P, Fung HKH, Lee CW, 807 Hagen W, Turonova B, Karius K, Bormel M, Zhang XJ, Muller CW, Schwab Y,

808 Mahamid J, Pfander B, Kosinski J, Beck M (2020) In-cell architecture of the nuclear 809 pore and snapshots of its turnover. Nature 586: 796-+

810 Beck M, Hurt E (2017) The nuclear pore complex: understanding its function through 811 structural insight. Nat Rev Mol Cell Biol 18: 73-89

812 Borah S, Thaller JD, Hakhverdyan Z, Rodriguez CE, Isenhour WA, Rout MR, King 813 CM, Lusk CP (2021) Heh2/Man1 may be an evolutionarily conserved sensor of NPC 814 assembly state. Mol Biol Cell 32

815 Carpenter AE, Jones TR, Lamprecht MR, Clarke C, Kang IH, Friman O, Guertin DA, 816 Chang JH, Lindquist RA, Moffat J, Golland P, Sabatini DM (2006) CellProfiler: image analysis software for identifying and quantifying cell phenotypes. Genome Biology 7 de Bruyn Kops A, Guthrie C (2001) An essential nuclear envelope integral membrane protein, Brr6p, required for nuclear transport. EMBO J 20: 4183-93

820 Doucet CM, Talamas JA, Hetzer MW (2010) Cell cycle-dependent differences in nuclear pore complex assembly in metazoa. Cell 141: 1030-41

822 Eisenberg D, Weiss RM, Terwilliger TC (1982) The Helical Hydrophobic Moment - a Measure of the Amphiphilicity of a Helix. Nature 299: 371-374

Ejsing CS, Sampaio JL, Surendranath V, Duchoslav E, Ekroos K, Klemm RW, Simons K, Shevchenko A (2009) Global analysis of the yeast lipidome by quantitative shotgun mass spectrometry. Proceedings of the National Academy of Sciences of the United States of America 106: 2136-2141 Sec63p, an endoplasmic reticulum membrane protein required for secretory protein translocation. Mol Cell Biol 12: 3288-96 Giddings TH, Jr., O'Toole ET, Morphew M, Mastronarde DN, Mclntosh JR, Winey M (2001) Using rapid freeze and freeze-substitution for the preparation of yeast cells for electron microscopy and three-dimensional analysis. Methods Cell Biol 67: 27-42 Gimenez-Andres M, Copic A, Antonny B (2018) The Many Faces of Amphipathic Helices. Biomolecules 8 Guthrie C, Fink GR (1991) Guide to yeast genetics and molecular biology. Meth Enzymol 194: 429-663 33: 119-24

840 Hodge CA, Choudhary V, Wolyniak MJ, Scarcelli JJ, Schneiter R, Cole CN (2010) 841 Integral membrane proteins Brr6 and Apq12 link assembly of the nuclear pore complex to lipid homeostasis in the endoplasmic reticulum. J Cell Sci 123: 141-51

843 Hu JJ, Shibata Y, Voss C, Shemesh T, Li ZL, Coughlin M, Kozlov MM, Rapoport TA, 844 Prinz WA (2008) Membrane proteins of the endoplasmic reticulum induce high845 curvature tubules. Science 319: 1247-1250 
Jang JH, Lee CS, Hwang D, Ryu SH (2012) Understanding of the roles of phospholipase $D$ and phosphatidic acid through their binding partners. Progress in Lipid Research 51: 71-81 Janke C, Magiera MM, Rathfelder N, Taxis C, Reber S, Maekawa H, MorenoBorchart A, Doenges G, Schwob E, Schiebel E, Knop M (2004) A versatile toolbox for PCR-based tagging of yeast genes: new fluorescent proteins, more markers and promoter substitution cassettes. Yeast 21: 947-962

Khmelinskii A, Blaszczak E, Pantazopoulou M, Fischer B, Omnus DJ, Le Dez G, Brossard A, Gunnarsson A, Barry JD, Meurer M, Kirrmaier D, Boone C, Huber W, Rabut G, Ljungdahl PO, Knop M (2014) Protein quality control at the inner nuclear membrane. Nature 516: 410-3

Knop M, Siegers K, Pereira G, Zachariae W, Winsor B, Nasmyth K, Schiebel E (1999) Epitope tagging of yeast genes using a PCR-based strategy: More tags and improved practical routines. Yeast 15: 963-972

Lone MA, Atkinson AE, Hodge CA, Cottier S, Martinez-Montanes F, Maithel S, MeneSaffrane L, Cole CN, Schneiter R (2015) Yeast Integral Membrane Proteins Apq12, Brl1, and Brr6 Form a Complex Important for Regulation of Membrane Homeostasis and Nuclear Pore Complex Biogenesis. Eukaryot Cell 14: 1217-27

Malsam J, Parisotto D, Bharat TAM, Scheutzow A, Krause JM, Briggs JAG, Sollner TH (2012) Complexin arrests a pool of docked vesicles for fast Ca2+-dependent release. Embo Journal 31: 3270-3281

Meijer M, Dorr B, Lammertse HCA, Blithikioti C, van Weering JRT, Toonen RFG, Sollner TH, Verhage M (2018) Tyrosine phosphorylation of Munc18-1 inhibits synaptic transmission by preventing SNARE assembly. Embo Journal 37: 300-320 Murphy R, Watkins JL, Wente SR (1996) GLE2, a Saccharomyces cerevisiae homologue of the Schizosaccharomyces pombe export factor RAE1, is required for nuclear pore complex structure and function. Molecular Biology of the Cell 7: 19211937

Onischenko E, Noor E, Fischer JS, Gillet L, Wojtynek M, Vallotton P, Weis K (2020) Maturation Kinetics of a Multiprotein Complex Revealed by Metabolic Labeling. Cell 183: $1785-+$

Onischenko E, Tang JH, Andersen KR, Knockenhauer KE, Vallotton P, Derrer CP, Kralt A, Mugler CF, Chan LY, Schwartz TU, Weis K (2017) Natively Unfolded FG Repeats Stabilize the Structure of the Nuclear Pore Complex. Cell 171: 904-917 e19 Otsuka S, Bui KH, Schorb M, Hossain MJ, Politi AZ, Koch B, Eltsov M, Beck M, Ellenberg $J(2016)$ Nuclear pore assembly proceeds by an inside-out extrusion of the nuclear envelope. Elife 5

Otsuka S, Ellenberg J (2018) Mechanisms of nuclear pore complex assembly - two different ways of building one molecular machine. FEBS Lett 592: 475-488

Otsuka S, Steyer AM, Schorb M, Heriche JK, Hossain MJ, Sethi S, Kueblbeck M, Schwab Y, Beck M, Ellenberg J (2018) Postmitotic nuclear pore assembly proceeds by radial dilation of small membrane openings. Nat Struct Mol Biol 25: 21-28

Ozbalci B, Boyaci IH, Topcu A, Kadilar C, Tamer U (2013) Rapid analysis of sugars in honey by processing Raman spectrum using chemometric methods and artificial neural networks. Food Chem 136: 1444-1452

Rampello AJ, Laudermilch E, Vishnoi N, Prophet SM, Shao L, Zhao C, Lusk CP, Schlieker C (2020) Torsin ATPase deficiency leads to defects in nuclear pore biogenesis and sequestration of MLF2. J Cell Biol 219

Romanauska A, Kohler A (2018) The Inner Nuclear Membrane Is a Metabolically Active Territory that Generates Nuclear Lipid Droplets. Cell 174: 700-715 e18 
896 Saitoh YH, Ogawa K, Nishimoto T (2005) Brl1p - a novel nuclear envelope protein required for nuclear transport. Traffic 6: 502-517

898 Sapay N, Guermeur Y, Deleage G (2006) Prediction of amphipathic in-plane 899 membrane anchors in monotopic proteins using a SVM classifier. BMC Bioinformatics 7: 255

902

Scarcelli JJ, Hodge CA, Cole CN (2007) The yeast integral membrane protein Apq12 potentially links membrane dynamics to assembly of nuclear pore complexes. $\mathrm{J}$ Cell Biol 178: 799-812 Smith SE, Slaughter BD, Unruh JR, Jaspersen SL (2016) Analysis of membrane proteins localizing to the inner nuclear envelope in living cells. Journal of Cell Biology 215: $575-590$

908 Sorger D, Daum G (2003) Triacylglycerol biosynthesis in yeast. Applied Microbiology and Biotechnology 61: 289-299 Souquet B, Freed E, Berto A, Andric V, Auduge N, Reina-San-Martin B, Lacy E, Doye V (2018) Nup133 Is Required for Proper Nuclear Pore Basket Assembly and Dynamics in Embryonic Stem Cells. Cell Reports 23: 2443-2454

Tagwerker C, Zhang H, Wang X, Larsen LS, Lathrop $\mathrm{RH}$, Hatfield GW, Auer B, Huang L, Kaiser P (2006) HB tag modules for PCR-based gene tagging and tandem affinity purification in Saccharomyces cerevisiae. Yeast 23: 623-32

Talamas JA, Hetzer MW (2011) POM121 and Sun1 play a role in early steps of interphase NPC assembly. Journal of Cell Biology 194: 27-37

918 Tamm T, Grallert A, Grossman EP, Alvarez-Tabares I, Stevens FE, Hagan IM (2011) Brr6 drives the Schizosaccharomyces pombe spindle pole body nuclear envelope insertion/extrusion cycle. J Cell Biol 195: 467-84 Tanguy E, Kassas N, Vitale N (2018) Protein-Phospholipid Interaction Motifs: A Focus on Phosphatidic Acid. Biomolecules 8

Tcheperegine SE, Marelli M, Wozniak RW (1999) Topology and functional domains of the yeast pore membrane protein Pom152p. J Biol Chem 274: 5252-8

Thaller DJ, Allegretti M, Borah S, Ronchi P, Beck M, Lusk CP (2019) An ESCRTLEM protein surveillance system is poised to directly monitor the nuclear envelope and nuclear transport system. Elife 8 Thaller DJ, Tong DQ, Marklew CJ, Ader NR, Mannino PJ, Borah S, King MC, Ciani B, Lusk CP (2021) Direct binding of ESCRT protein Chm7 to phosphatidic acid-rich membranes at nuclear envelope herniations. Journal of Cell Biology 220 Thiam AR, Farese RV, Jr., Walther TC (2013) The biophysics and cell biology of lipid droplets. Nat Rev Mol Cell Biol 14: 775-86 Ungricht R, Kutay U (2017) Mechanisms and functions of nuclear envelope remodelling. Nat Rev Mol Cell Biol 18: 229-245 van Leeuwen J, Pons C, Tan GH, Wang JSZ, Hou J, Weile J, Gebbia M, Liang W, Shuteriqi E, Li ZJ, Lopes M, Usaj M, Lopes AD, van Lieshout N, Myers CL, Roth FP, Aloy P, Andrews BJ, Boone C (2020) Systematic analysis of bypass suppression of essential genes. Molecular Systems Biology 16 Wang N, Clark LD, Gao Y, Kozlov MM, Shemesh T, Rapoport TA (2021) Mechanism of membrane-curvature generation by ER-tubule shaping proteins. Nat Commun 12 Weber T, Zemelman BV, McNew JA, Westermann B, Gmachl M, Parlati F, Sollner TH, Rothman JE (1998) SNARE dependent membrane fusion. Mol Biol Cell 9: 331a$331 \mathrm{a}$ complex assembly by ESCRT-III/Vps4. Cell 159: 388-401 
946 Wente SR, Blobel G (1993) A temperature-sensitive NUP116 null mutant forms a

947 nuclear envelope seal over the yeast nuclear pore complex thereby blocking 948 nucleocytoplasmic traffic. J Cell Biol 123: 275-84

949 Winey M, Hoyt MA, Chan C, Goetsch L, Botstein D, Byers B (1993) NDC1: a nuclear 950 periphery component required for yeast spindle pole body duplication. J Cell Biol 951 122: 743-751 Winey M, Yarar D, Giddings TH, Jr., Mastronarde DN (1997) Nuclear pore complex number and distribution throughout the Saccharomyces cerevisiae cell cycle by three-dimensional reconstruction from electron micrographs of nuclear envelopes. Mol Biol Cell 8: 2119-32

956 Zhang W, Neuner A, Rüthnick D, Sachsenheimer T, Lüchtenborg C, Brügger B, 957 Schiebel E (2018) Brr6 and Brl1 locate to nuclear pore complex assembly sites to 958 promote their biogenesis. J Cell Biol 217: 877-894 Key Role of Polyphosphoinositides in Dynamics of Fusogenic Nuclear Membrane Vesicles. Plos One 6 Zhukovsky MA, Filograna A, Luini A, Corda D, Valente C (2019) Phosphatidic acid in membrane rearrangements. Febs Letters 593: 2428-2451 


\section{Figure legends}

991

992

993

994

995

996

997

998

999

1000

1001

1002

1003

1004

1005

1006

1007

1008

1009

1010

1011

1012

1013

1014

1015

1016

1017

1018

1019

1020

1021

1022

1023

1024

\section{Figure 1. Apq12 contains an amphipathic helix in the luminal domain}

(A) Domain organization of Apq12. The first transmembrane (TM1) domain, an AaH (blue) and the second transmembrane (TM2) domain are indicated. (B) Heliquest predictions of the $\mathrm{AaH}, \mathrm{AaH}-\mathrm{ah}$, and $\mathrm{AaH}^{\mathrm{F5DI6RV} 9 \mathrm{~N}}$ helices along with their hydrophobic moments. The amino acids marked in green indicate amino acid changes introduced in $\mathrm{AaH}$ in order to reduce the hydrophobic moment. (C) Binding of Atto488 labelled synthetic $\mathrm{AaH}$ and $\mathrm{AaH}$-ah peptides to Atto647 labelled GUVs, in vitro. DMSO and Atto488 dye are used as controls. Scale bar: 5 $\mu \mathrm{m}$. (D) Liposomes with different lipid compositions were titrated against Atto488 labeled $\mathrm{AaH}$ and $\mathrm{AaH}$-ah peptides and the Atto488 dye as control. The data were normalized to the amount of bound peptide and the $\mathrm{K}_{\mathrm{D}}$ value of $\mathrm{AaH}$ peptide-liposome interaction was calculated from the Hill equation ( $\left.P M: K_{D}=58.90 \pm 5.35 \mu \mathrm{M}, N E: K_{D}=15.90 \pm 0.91 \mu \mathrm{M}\right)$. The $\mathrm{AaH}-\mathrm{ah}$ peptide and the Atto488 dye (together with NE lipids; same result was obtained with PM lipids) show no or only very weak MST signals. Values are given as means \pm S.E.M, $\mathrm{n}=3$.

Figure 2. The AaH of Apq12 is located in the perinuclear space and does not influence the subcellular localization and topology

(A) Growth test of WT APQ12, apq12L, apq12-ah, apq12 F5DI6RV9N mutants at the indicated temperatures. 10-fold serial dilutions were spotted onto YPAD plates. (B) Localization of yeGFP-Apq12 and yeGFP-apq12-ah were analyzed by fluorescence microscopy. Scale bar: $5 \mu \mathrm{m}$. (C) yeGFP-Apq12 and yeGFP-apq12-ah localization by immuno-EM. Gold particles $(10 \mathrm{~nm})$ indicate the localization of yeGFP-Apq12 and yeGFP-apq12-ah at the NE and ER. The rectangles indicate the enlargements that are shown underneath. Abbreviation: $N$ : nucleus. Scale bars: $200 \mathrm{~nm}$ and enlargements $50 \mathrm{~nm}$. (D) Strains carrying C- and Nterminal fusions of Apq12 and Apq12-ah with GFP $_{1-10}$ were imaged to check for reconstitution of GFP with GFP $_{11}$ from GFP 11 -mCherry-Scs2TM (ER reporter; GFP 11 in the cytoplasm) and GFP $_{11}$-mCherry-PUS1 (nuclear reporter). Mps3-GFP ${ }_{1-10}$ is used as a negative control. Scale bar: $5 \mu \mathrm{m}$. (E) Localization and topology model for Apq12.

\section{Figure 3. Phenotypes of apq12 $\Delta$ and apq12-ah cells}

(A) EM analysis of $A P Q 12$ WT cells incubated at the indicated temperatures. (B) EM analysis of apq12 12 mutants incubated at the indicated temperatures. The arrows point towards the phenotype that is indicated by the colour code encircling the picture. (C) 
1025 Quantification of phenotypes from (B). Cells $\left(n=44 / 16^{\circ} \mathrm{C}, 40 / 23^{\circ} \mathrm{C}\right.$ and $\left.57 / 37^{\circ} \mathrm{C}\right)$ were 1026 analyzed per temperature. (D) EM analysis of apq12-ah mutants grown at the indicated 1027 temperature. The arrows point towards the phenotype that is indicated by the colour code 1028 encircling the picture. (E) Quantification of phenotypes from (D). Cells ( $\left.n=27 / 16^{\circ} \mathrm{C}, 3^{39}\right)^{2} 3^{\circ} \mathrm{C}$ 1029 and $28 / 37^{\circ} \mathrm{C}$ ) were analyzed per temperature. (A, B and D) Abbreviation: N, nucleus. Scale 1030 bars: (A) $100 \mathrm{~nm}$; (B, D) $500 \mathrm{~nm}$.

1031

1032

Figure 4. Overexpression of $A P Q 12$ causes the mislocalization of ER proteins

1033 (A) Overexpression of $A P Q 12$ is toxic for the cells. WT cells with the vector control,

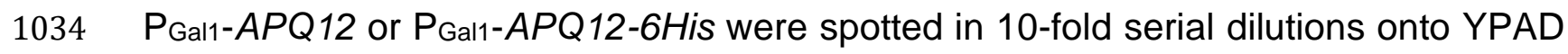
1035 (Glu) and YARaf/Gal (Gal/Raf) plates at 30․ (B) Of the APQ12, BRL1, BRR6 1036 module only $A P Q 12$ overexpression is toxic. WT cells with the vector control, PGal11037 APQ12, $\mathrm{P}_{\mathrm{Gal1}}-B R R 6$ and $\mathrm{P}_{\mathrm{Gal1}}-B R L 1$ were spotted in 10-fold serial dilutions onto YPAD (Glu) and YARaf/Gal (Gal/Raf) plates at 30ㄷ. (C) Overexpression of APQ12 causes mislocalization of yeGFP-Brl1. Cells with either the vector control (Fig. S3C) or PGal1-APQ12-6His were incubated with galactose for the indicated times. The boxed cell at $1 \mathrm{~h}$ is a two-fold enlargement of the selected cell. (D) Line scan along the NE of a PGal1-APQ12-6His yeGFP-BRL1 NUP85-tdTomato cell (enlarged boxed cell in $(\mathrm{C})$ ) incubated for $1 \mathrm{~h}$ with galactose. It shows that yeGFP-Brl1 and Nup85tdTomato are localized on separate domains along the NE. (E) Immunoblot of PGal1APQ12-6His yeGFP-BRL1 NUP85-tdTomato cells. The pGal1 promoter was induced by the addition of galactose $(\mathrm{t}=0)$. Samples were taken after the indicated times. Tub2 is a loading control. Apq12-6His was detected by anti-His antibodies. (F) Overexpression of $A P Q 12$ causes mislocalization of yeGFP-Brr6 and Nup85tdTomato. Cells with either the vector control (Fig. S3D) or PGal1-APQ12-6His were incubated with galactose for the indicated times. The boxed cell at $1 \mathrm{~h}$ is a two-fold enlargement of the selected cell. (G) Line scan along the NE of a PGal1-APQ12-6His yeGFP-BRR6 NUP85-tdTomato cell (enlarged cell in $(\mathrm{F})$ ) incubated for $1 \mathrm{~h}$ with

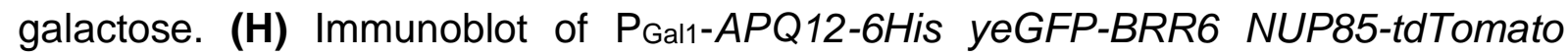
cells. The pGal1 promoter was induced by the addition of galactose $(t=0)$. Samples were taken after the indicated times. Tub2 is a loading control. (I, J) Overexpression of $A P Q 12$ causes mislocalization of the ER protein Sec63-yeGFP (I) and Ole1yeGFP $(\mathbf{J})$. Cells with either the vector control or $\mathrm{P}_{\text {Gal1-APQ12-6His were incubated }}$ with galactose for the indicated time. (C, F, I and J) Scale bars: $5 \mu \mathrm{m}$. 
1062 (A) Inactivation of the amphipathic $\alpha$-helix of Apq12 reduces overexpression toxicity. Growth

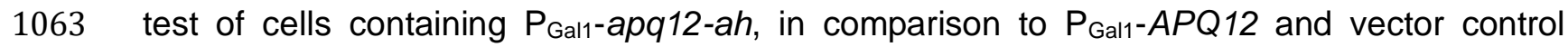
1064 cells. 10-fold serial dilutions of cells were spotted onto glucose (Glu) and galactose/raffinose 1065 (Gal/Raf) plates at 30ㄷ. (B-E) Impact of $A P Q 12$ and apq12-ah overexpression on (B, C) 1066 yeGFP-BRL1 NUP85-tdTomato and (D, E) yeGFP-BRR6 NUP85-tdTomato cells. (C, E) are 1067 quantifications of the maximum GFP intensity of individual cells from (B) and (D) over time. 1068 Two independent experiments were performed, and more than 60 cells were analyzed per 1069 experiment for each time point. Values are given as means $+/-\mathrm{SD}$. $t$-test ${ }^{* * * *} \mathrm{p}<0.0001$. (B, D) 1070 Scale bars: $5 \mu \mathrm{m}$. (A) EM analysis of WT cells expressing $\mathrm{P}_{\mathrm{Gal}}$-APQ12-6His (left) or $\mathrm{P}_{\mathrm{Gal}}$-apq12-ah-6His (right) by the addition of galactose for $0.5 \mathrm{~h}, 1 \mathrm{~h}$, and $3 \mathrm{~h}$. Arrows are explained in the result section; red asterisks indicate ONMs encircled vesicles containing granular material. Abbreviation: $\mathrm{N}$, nucleus. Scale bar: $200 \mathrm{~nm}$. (B) Quantification of phenotypes from (A). $n=25$ cells were analyzed for each time point and strain. (C) $P_{\text {Gal1- }} A P Q 12$ was overexpressed for $3 \mathrm{~h}$ by the addition of galactose. Top panel: cells expressing yeGFP-Brl1 along with a nuclear marker NLS-mRFP. Bottom panel: cells expressing yeGFP tagged ribosomal subunit Rpl25, and an ER luminal marker dsRED-HDEL. Scale bar: $3 \mu \mathrm{m}$ (D) Model for the formation of membrane structures in response to $A P Q 12$ overexpression. See result for details. (A) Localization of the cytoplasmic PA sensors (Q2-mCherry) in response to $\mathrm{P}_{\text {Gal1 }}-A P Q 12$

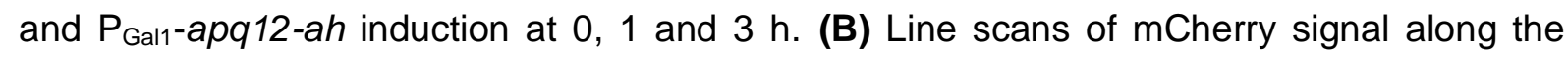
indicated lines across the cells in (A). Arrowheads mark the peaks corresponding to the cytoplasmic membrane (C) and NE (N). (C) Quantification of data from (A) showing percentage of cells with nuclear rim localization after $3 \mathrm{~h}$ of $\mathrm{P}_{\mathrm{Gal1}}-A P Q 12$ induction. 100 cells carrying either $\mathrm{P}_{\mathrm{Gal1}}-\mathrm{APQ12}$ or $\mathrm{P}_{\mathrm{Gal1}}$-apq12-ah from one representative experiment were analyzed for nuclear rim localization of the PA sensor. (D) Localization of the nuclear PA

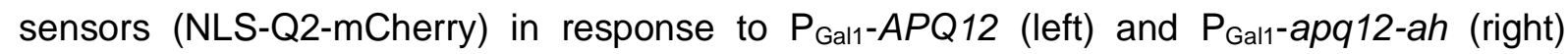
induction at 0,1 and $3 \mathrm{~h}$. (E) Line scans of mCherry signal along the indicated lines across the nuclei in (D). Arrowheads mark the peaks corresponding to the NE (N). (F) Quantification of data from (D) showing percentage of cells with nuclear rim localization done as in (C). (A, 
1099 (A) yeGFP-Apq12 localization in WT, td-brr6 and td-brl1 cells carrying the NPC marker 1100 NUP85-tdTomato. Cells were incubated for $0 \mathrm{~h}$ and $3 \mathrm{~h}$ with $\mathrm{P}_{\text {Gal1- }}$ UBR1 induction at $37^{\circ} \mathrm{C}$.

1101 (B) Line scans along the NE of cells shown in the insets in (A). (C) Scatterplot with Pearson 1102 correlation coefficient ( $r$ ) of GFP and mCherry fluorescence intensities (in arbitrary units, a.u.) 1103 along the nuclear rim of 5 cells. (D) yeGFP-Apq12-ah localization in WT, td-brr6 and td-brl1 1104 cells carrying the NPC marker NUP85-tdTomato. Cells were incubated for $0 \mathrm{~h}$ and $3 \mathrm{~h}$ with $1105 \mathrm{P}_{\text {Gal1 }}-U B R 1$ induction at $37^{\circ} \mathrm{C}$. (E) Line scans along the NE of cells shown in the insets in (D). 1106 (F) Scatterplot with Pearson correlation coefficient ( $r$ ) of GFP and mCherry fluorescence 1107 intensities (in arbitrary units, a.u.) along the nuclear rim of 5 cells. (A, D) Scale bars: $5 \mu \mathrm{m}$. 1108 (G) $t d$-brl1 and $t d$-brr6 cells with yeGFP-APQ12 or yeGFP-apq12-ah were incubated for $3 \mathrm{~h}$ 1109 under restrictive conditions (galactose, $37^{\circ} \mathrm{C}$ ) to induce degradation of Brl1 and Brr6 and the 1110 formation of herniations. Fixed and embedded cells were analyzed for localization of yeGFP1111 Apq12 and yeGFP-Apq12-ah by immuno-EM using GFP antibodies and $10 \mathrm{~nm}$ gold labelled 1112 protein A. Blue arrowheads indicate the localization of $10 \mathrm{~nm}$ gold particles. Scale bars: 100 $1113 \mathrm{~nm}$.

\section{Figure 9. Interaction of Brl1 and Brr6 is regulated by AaH of APQ12}

1116 (A) Immunoblot showing endogenous levels of Brl1 in WT APQ12, apq12 1 and apq12-ah 1117 backgrounds, using anti-Brl1 antibody. Tub2 is loading control. (B) Quantification of Brl1 from 1118 (A) normalized to Tub2. Error bars are $S D, n=3 .{ }^{* *} \mathrm{p}<0.01$. (C) Immunoblot showing levels of 1119 Brr6-yeGFP in WT (APQ12) and apq12-ah backgrounds using anti-GFP antibody. Tub2 is 1120 loading control. (D) Quantification of Brr6 from (C) normalized to Tub2. Error bars are SD, $n$ $1121=3 .{ }^{* * *} \mathrm{p}<0.001$. (E) Localization of Brr6-yeGFP and Brl1-yeGFP in WT APQ12 (left) and 1122 apq12-ah cells (right) and corresponding line scans along NE of cells shown in the insets. 1123 Scale bar: $5 \mu \mathrm{m}$. (F) Co-IP of Apq12, Brl1 and Brr6. Brr6-yeGFP was immunoprecipitated 1124 with GFP antibodies. Apq12-6HA was detected with anti-HA and Brl1 with Brl1 antibodies.

1125 (G) Quantification of the ratio between Apq12 and Brr6-yeGFP and, Brl1 and Brr6-yeGFP in 1126 WT $A P Q 12$ and apq12-ah backgrounds. Error bars are SD, $\mathrm{n}=3$; t-test; * $\mathrm{p}<0.05$.

1129 (A) The diameter of liposomes of PM or NE lipids was measured by DLS with 20 acquisitions 1130 at room temperature before and after dialysis with the MST buffer. (B) Binding of AaH and $1131 \mathrm{AaH}$-ah peptides to liposomes measured by float-up. The data were compared to the input. 1132 Values are given as means \pm S.E.M $\left(n=3\right.$; t-test: $\left.{ }^{* *} p<0.01\right)$. (C) Quantification of data from 
1133 Fig. 2C. Number of analyzed gold particles for each category is given in the figure. (D) 1134 Immuno-EM images of cells with and without yeGFP-Apq12 and yeGFP-Apq12-ah at NPCs.

1135 For yeGFP-Apq12 and yeGFP-Apq12-ah 12 and 33 NPCs were analyzed. Scale bars: 50 $1136 \mathrm{~nm}$. (E) Analysis of the membrane topology of Apq12. The indicated yeast cells were 1137 analyzed by immunoblotting for the biotinylation of the $\mathrm{HBH}$ tag for cytoplasmic or nuclear 1138 localization. $\mathrm{N}$ and $\mathrm{C}$ terminally tagged Pom152 is used as a control.

1140 Figure S2. Lipid analysis of WT and apq12 $\triangle$ cells

1141 Lipids were extracted from APQ12 WT, apq12 1 and apq12-ah cells incubated at the 1142 indicated temperatures and subsequently analyzed by nano-ESI-MS/MS. Samples were 1143 taken after $2 \mathrm{~h}$ at $37^{\circ} \mathrm{C}, 4 \mathrm{~h}$ at $30^{\circ} \mathrm{C}$ and $18 \mathrm{~h}$ at $16^{\circ} \mathrm{C}$. (A) Plots of lipid species in mol\% of 1144 measured lipids. (B) Plots of functional categories of lipids; glycerophospholipids and 1145 glycerolipids (GPL), sphingolipids (SP), sterols (ST), and storage lipids. (C) Plots showing 1146 the number of double bonds in GPL. (D) Plots showing the chain lengths of the GPL. $n=3$. 1147 Values are given as means + SD. t-test ${ }^{*} p<0.05,{ }^{* *} p<0.01,{ }^{* *} p<0.001,{ }^{* * *} p<0.0001$.

1149 Figure S3. Overexpression of $A P Q 12-6 H i s$ has a similar impact as that of $A P Q 12$

1150 (A, B) Induction of $\mathrm{P}_{\text {Gal1 }}-A P Q 12$ caused the same phenotypes in yeGFP-BRL1 NUP85tdTomato $(\mathrm{A})$ and yeGFP-BRR6 NUP85-tdTomato $(\mathrm{B})$ cells as seen for $\mathrm{P}_{\mathrm{Gal1}}-\mathrm{APQ12-6His.}$ (C, D) Cells with the vector control p425- $P_{\text {Gal1 }}$ show no impact on yeGFP-Brl1, yeGFP-Brr6 and Nup85-tdTomato upon addition of galactose $(\mathrm{t}=0)$. (E) Live-cell imaging of yeGFP-Brl1 and Nup85-tdTomato with induction of $\mathrm{P}_{\text {Gal1- }}-A P Q 12$ by addition of galactose $(\mathrm{t}=0)$. (F) Livecell imaging of yeGFP-Brr6 and Nup85-tdTomato with induction of $\mathrm{P}_{\text {Gal1 }}-A P Q 12$ by addition of galactose $(t=0)$. (A-F) Scale bars: $5 \mu \mathrm{m}$.

Figure S4. Overexpression of APQ12-6His weakly affects localization of the INM protein Asi3 but strongly affects the ER luminal marker HDEL

1160 (A, B) Impact of $\mathrm{P}_{\text {Gal1 }}$-APQ12 overexpression on the INM protein Asi3-yeGFP $(\mathrm{A})$ and the ER marker dsRed-HDEL (B). Induction of $\mathrm{P}_{\text {Gal1 }}-A P Q 12$ was triggered by the addition of galactose $(\mathrm{t}=0)$. The arrows in $(\mathrm{A})$ indicate small enrichments of Asi3-yeGFP at the NE after $3 \mathrm{~h}$ of

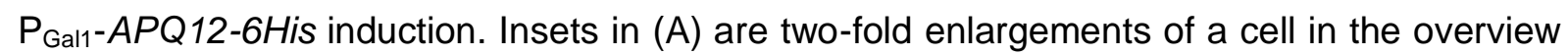
marked by an asterisk. (A, B) BF: brightfield. Scale bars: (A, B) $5 \mu \mathrm{m}$.

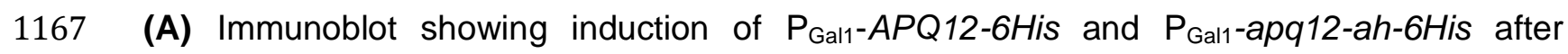
1168 addition of galactose. His antibodies detected Apq12-6His and Apq12-ah-6His. Tub2 is 1169 loading control. (B) EM analysis of $\mathrm{P}_{\mathrm{Gal1}}-\mathrm{APQ12}-6$ His cells at $\mathrm{t}=0$ and vector control pGal1 
1170 cells at $t=1$ of galactose addition. Scale bars: $250 \mathrm{~nm}$. (C) Live-cell imaging of Apq12-yeGFP 1171 (left) and Apq12-ah-yeGFP (right) in combination with Nup85-tdTomato upon galactose ( $\mathrm{t}=0$ )

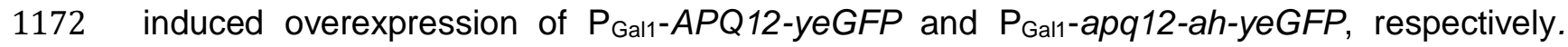
1173 White arrowheads indicate NE extensions. Scale bars: $3 \mu \mathrm{m}$. (D) Immuno-EM localization of 1174 Apq12-yeGFP in cells expressing $\mathrm{P}_{\mathrm{Gal1} 1}-A P Q 12-y e G F P$ for 0.5 and $3 \mathrm{~h}$ induction by addition 1175 of galactose. The $10 \mathrm{~nm}$ gold particles indicate localization of Apq12-yeGFP. The ochre 1176 rectangles on the left illustrate the magnifications that are shown on the upper right. The 1177 cartoons on the lower right are schematic views on the ER/ONM of the EM pictures in the top 1178 right. Gold particles indicating the localization of Apq12-yeGFP are shown in the cartoons. 1179 Abbreviation: N, nucleus. Scale bars: $250 \mathrm{~nm}$ and enragements $50 \mathrm{~nm}$.

Figure S6. APQ12 overexpression induces lipid metabolism flow from storage lipids towards membrane lipids

1183 (A-D) Samples were prepared from cells incubated at $30^{\circ} \mathrm{C}$ and expressing $\mathrm{P}_{\text {Gal1 }}-A P Q 12-$ 11846 His and $\mathrm{P}_{\mathrm{Gal1}}$-Apq12-ah-6His upon addition of galactose for indicated times, and lipids were extracted for subsequent analysis by nano-ESI-MS/MS. (A) Plots of lipid species in mol\% of measured lipids. (B) Plots of functional categories of lipids; glycerophospholipids and glycerolipids (GPL), sphingolipids (SP), sterols (ST), and storage lipids. (C) Plots showing the number of double bonds in GPL. (D) Plots showing the chain lengths of the GPL. $n=3$. Values are given as means + SD. t-test ${ }^{*} p<0.05,{ }^{* *} p<0.01$.

1192 (A) Localization of yeGFP-Apq12 and yeGFP-Apq12-ah was analyzed in td-brl1 and td-brr6 1193 cells by immuno-EM as described in Fig. 8G. The $10 \mathrm{~nm}$ gold particles indicate localization of 1194 yeGFP-Apq12 and yeGFP-Apq12-ah. Scale bars: $200 \mathrm{~nm}$, enlargements $100 \mathrm{~nm}$. (B) 1195 Quantification of data from Fig. 8G and Fig. S7A. Gold particles reflecting localization of 1196 yeGFP-Apq12 and yeGFP-Apq12-ah at herniations were classified as indicated in the figure. 1197 Localization of 31 gold particles was analyzed for td-brr6 APQ12-yeGFP, 38 for td-brl1 1198 APQ12-yeGFP, 37 for td-brr6 apq12-ah-yeGFP and 33 for td-brl16 apq12-ah-yeGFP cells.

1199 (C) Graph quantifying the number of particles, reflecting yeGFP-Apq12 and yeGFP-Apq121200 ah, per $\mu \mathrm{m}$ of the NE. 24 gold particles were analyzed for yeGFP-APq12, 32 for td-brl1 yeGFP-APQ12 and 39 for td-brr6 yeGFP-APQ12 cells. 


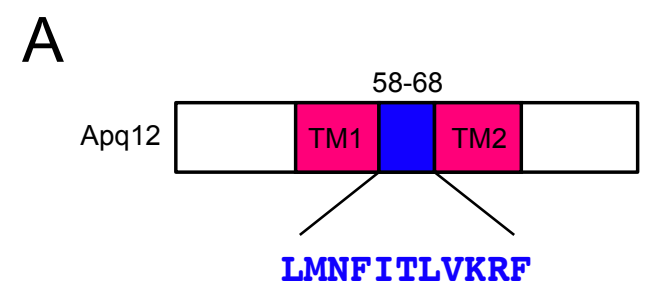

B

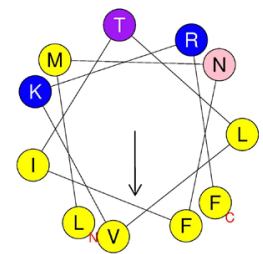

$\mathrm{AaH}$

0.595

LMNF ITLVKRF

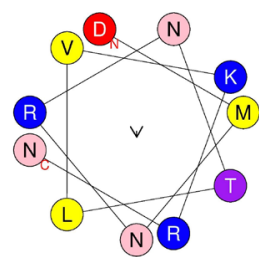

AaH-ah

0.054

DMNRNTLVKRN

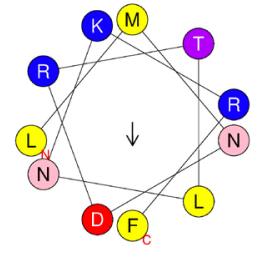

$\mathrm{AaH}^{\text {F5DI6RV9N }}$

0.222

LMNDRTLNKRF
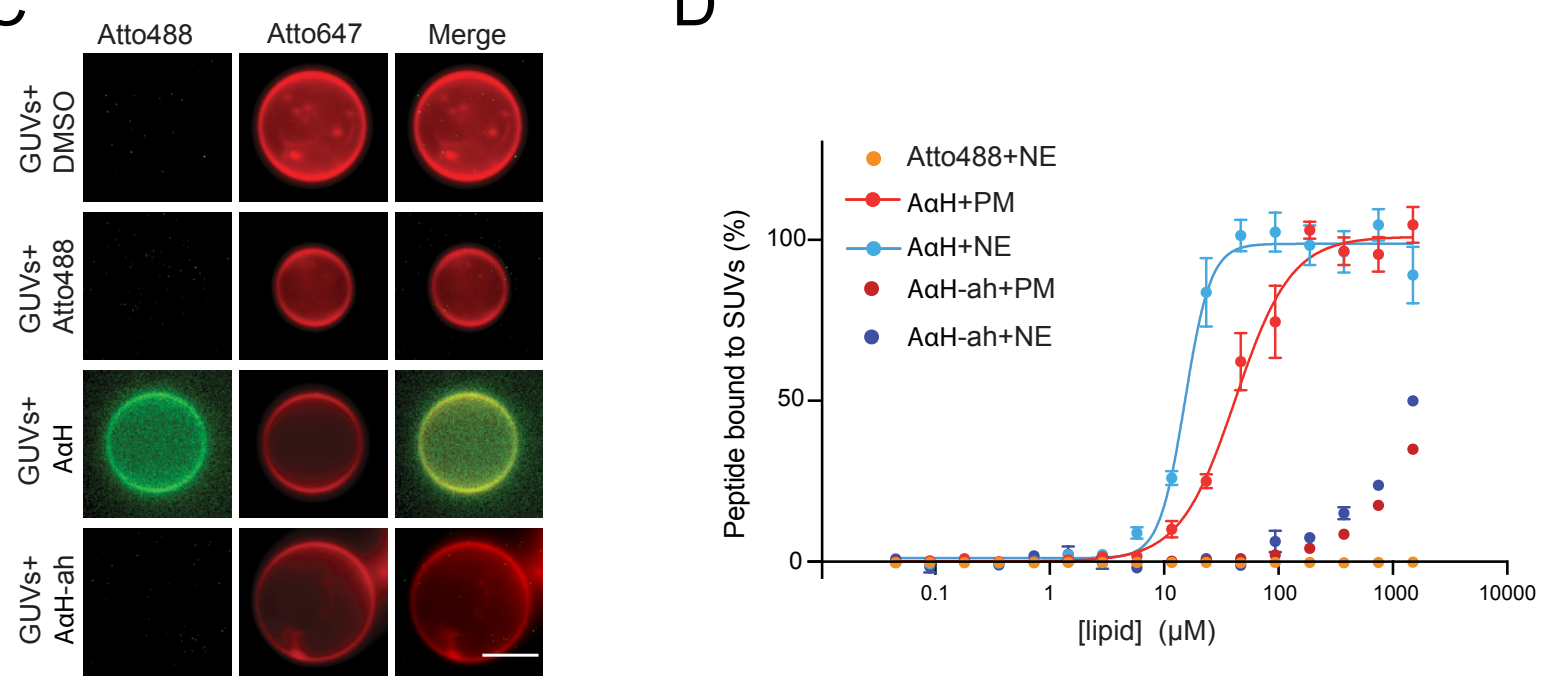

D lipid] $(\mu \mathrm{M})$ 


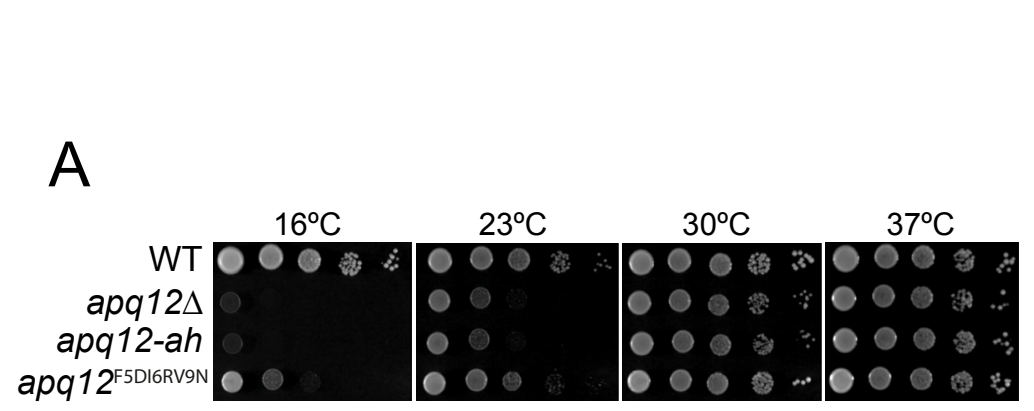

B

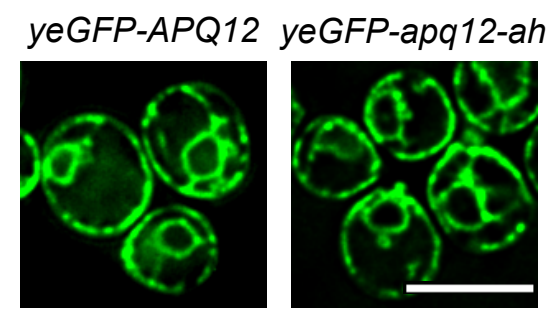

\section{C}
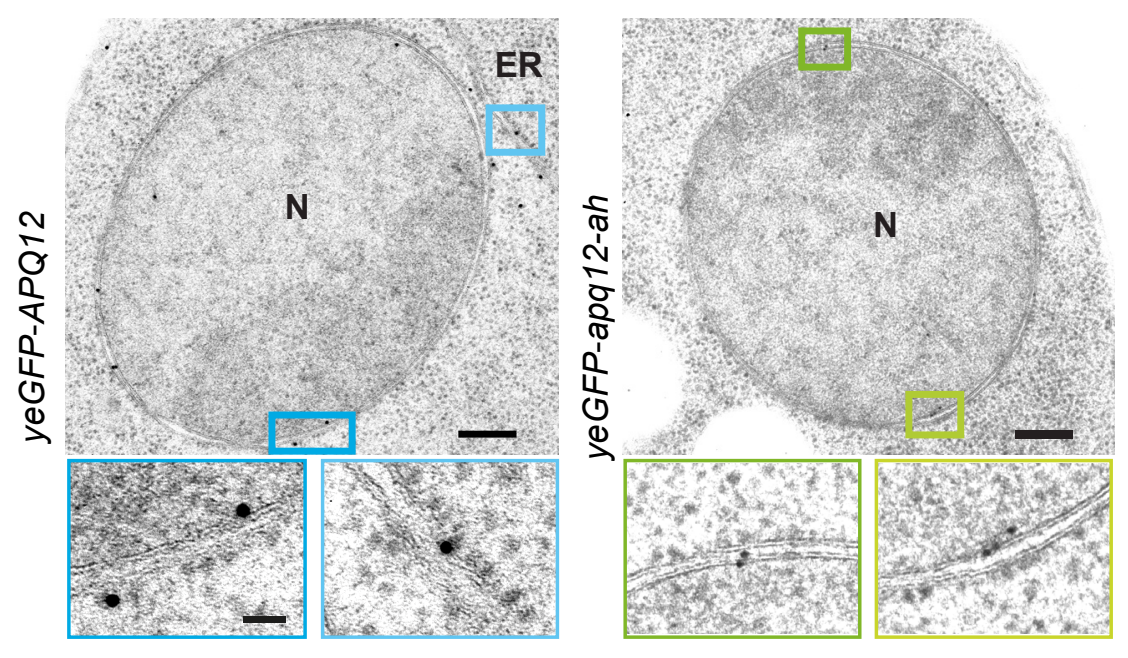

$E$

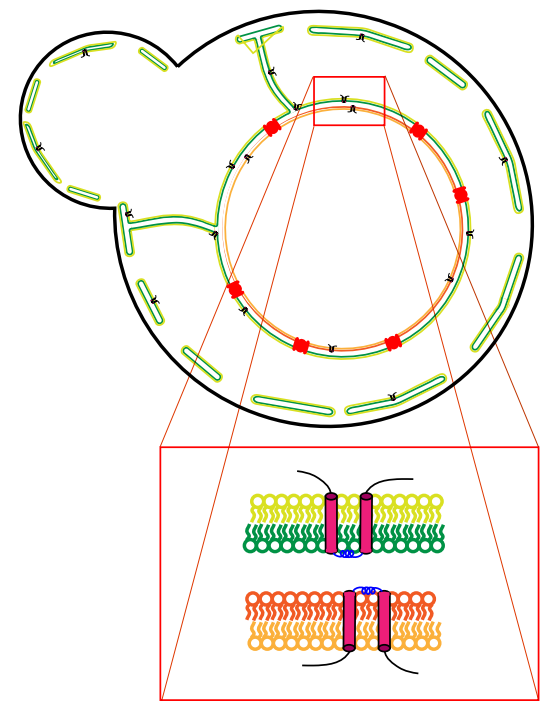

$\mathrm{D}$
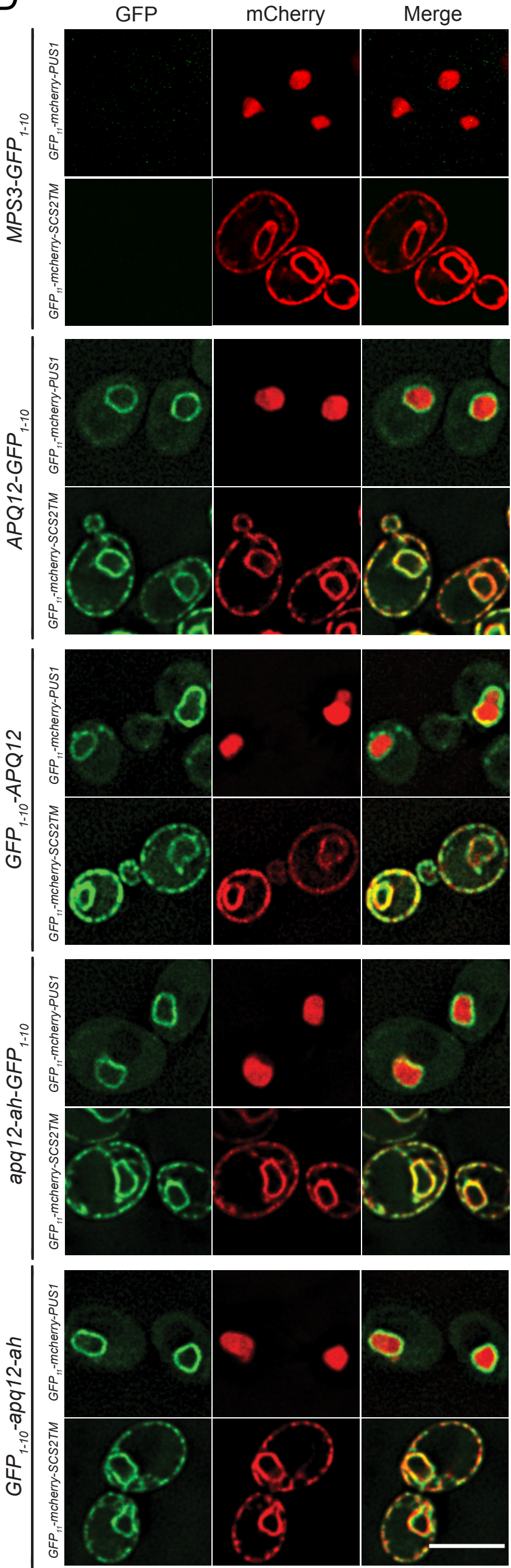
A $\quad 16^{\circ} \mathrm{C}$

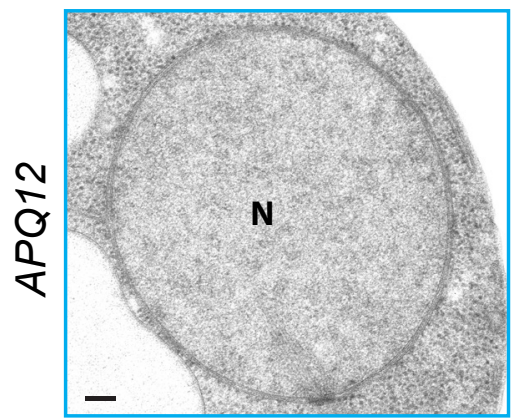

$37^{\circ} \mathrm{C}$

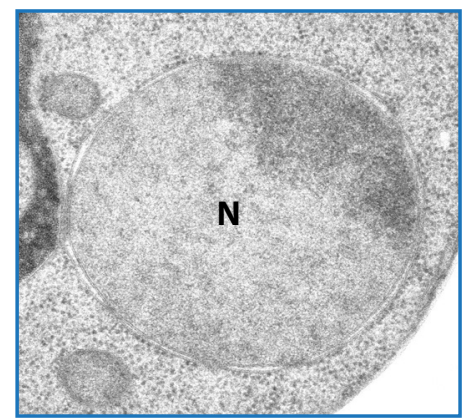

B
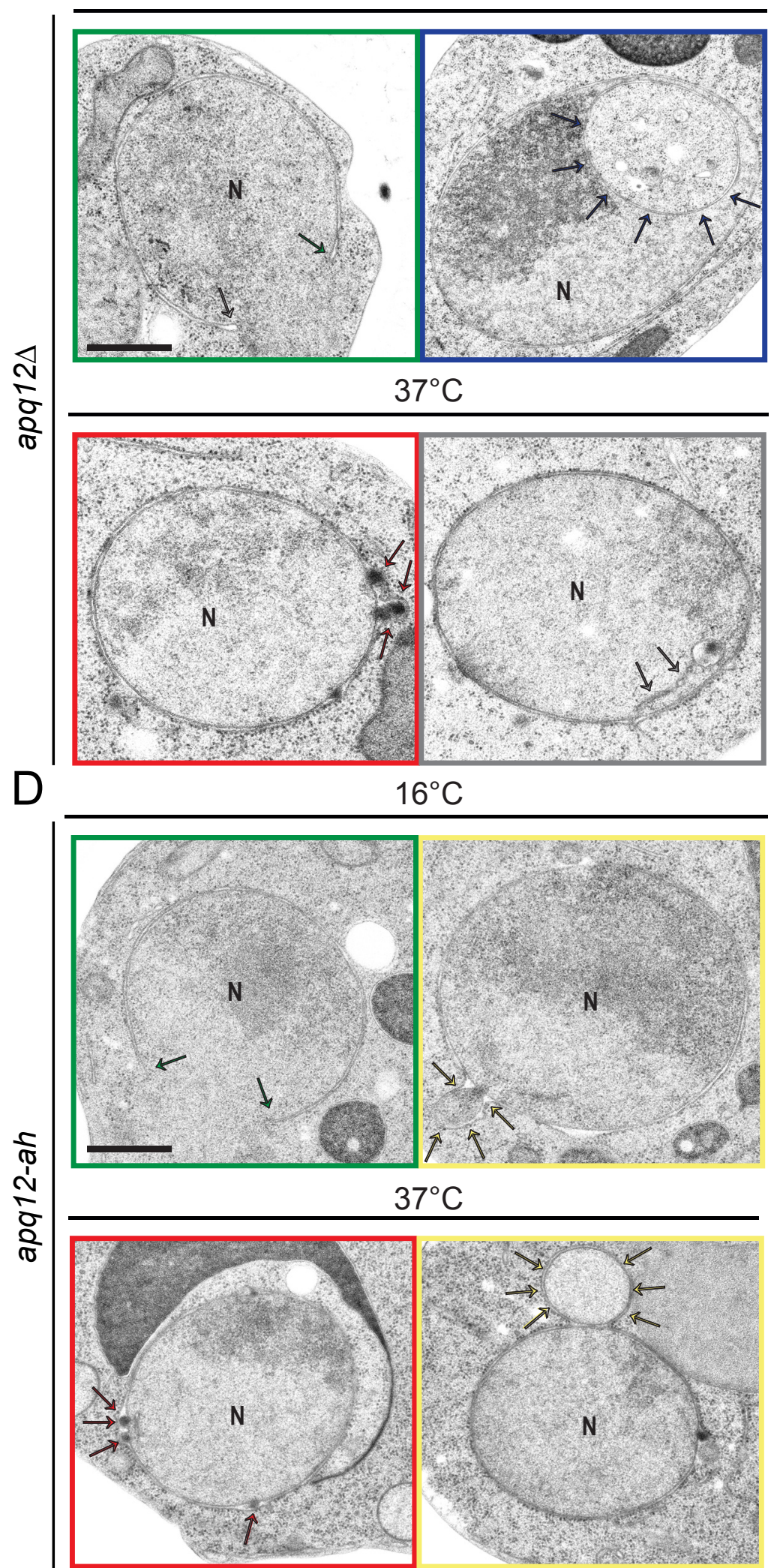

C

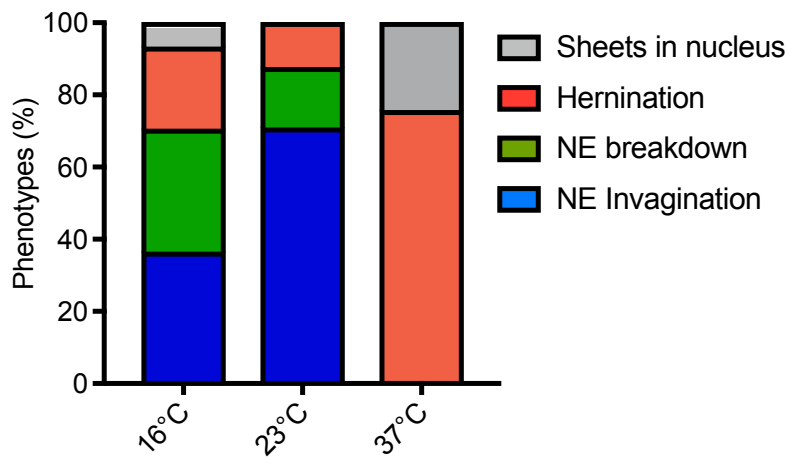

E

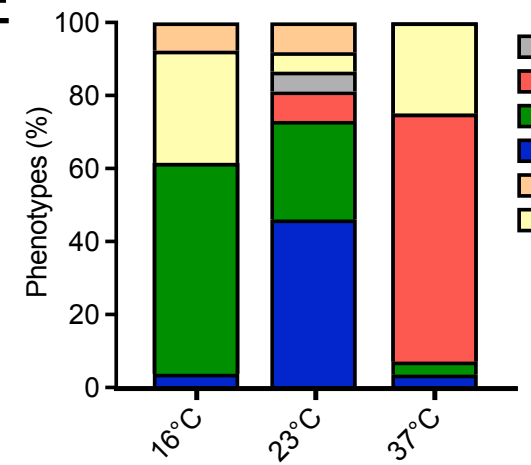

Sheets in nucleus Herniations

NE breakdown

NE invaginations

Extended ER

Extrusions in NE 
A

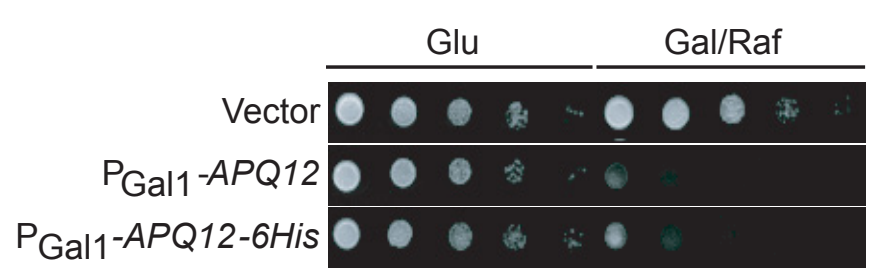

C

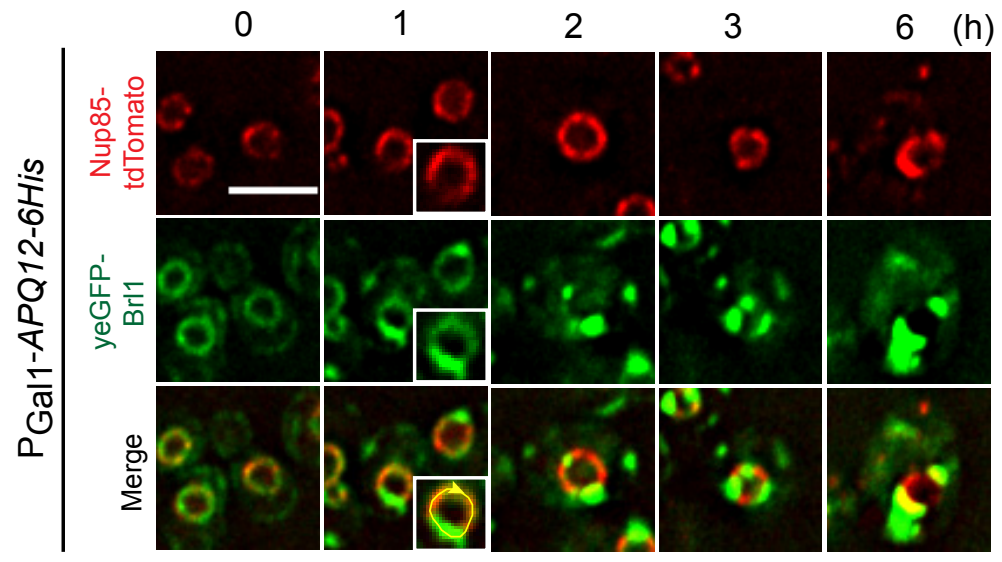

F

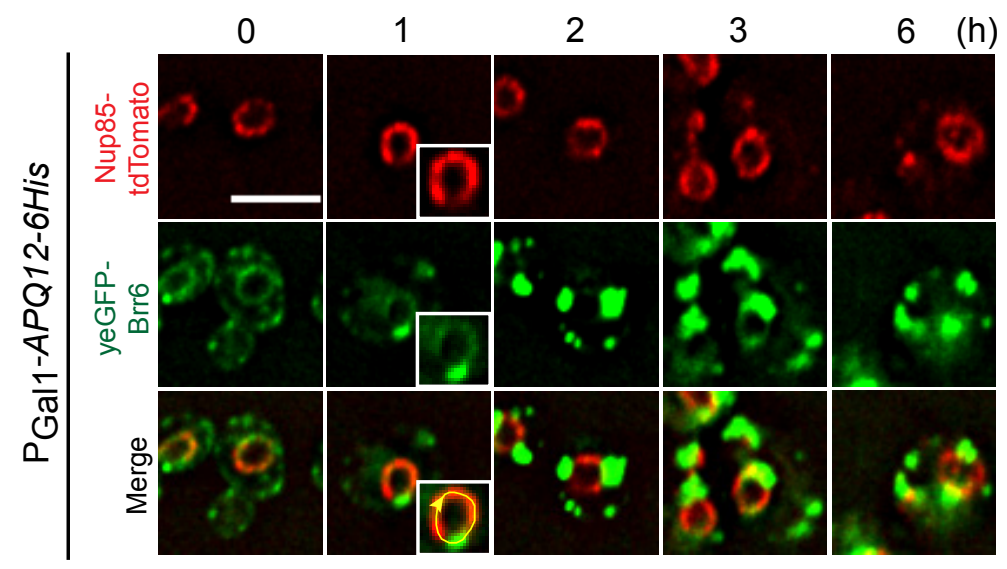

B

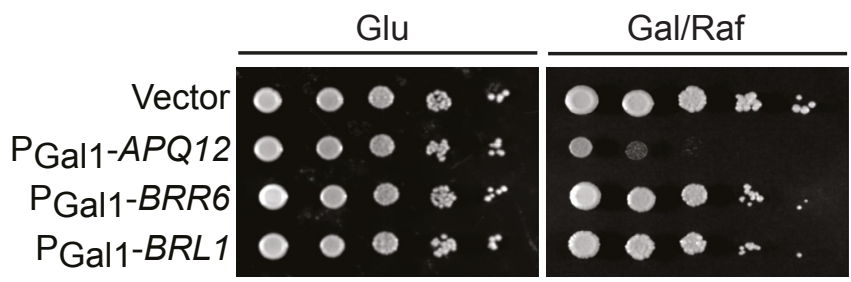

E
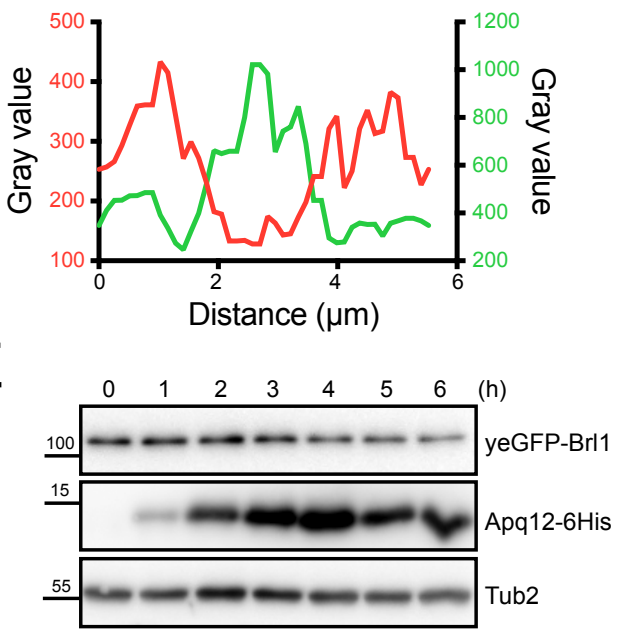

G

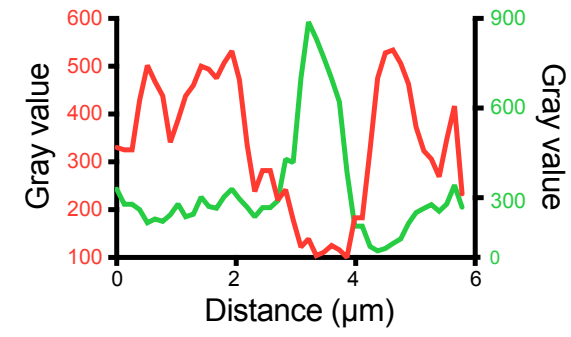

$\mathrm{H}$

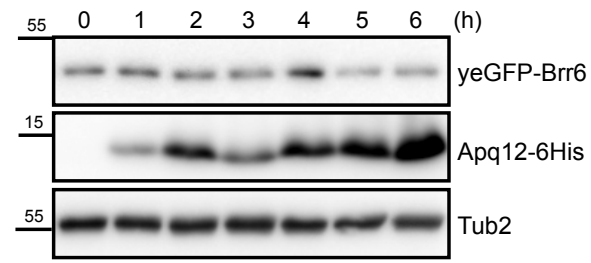

I

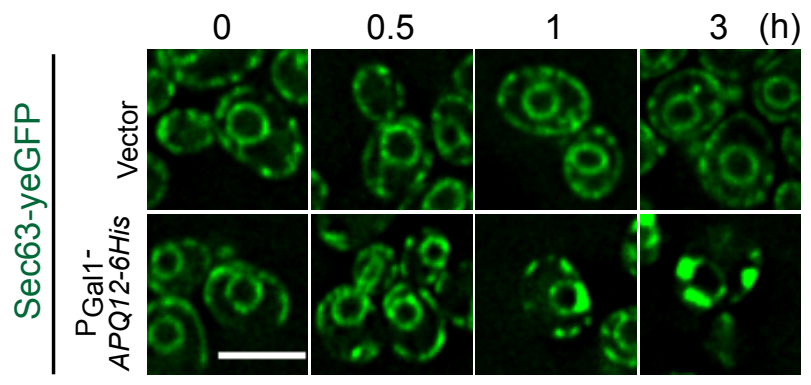

$J$

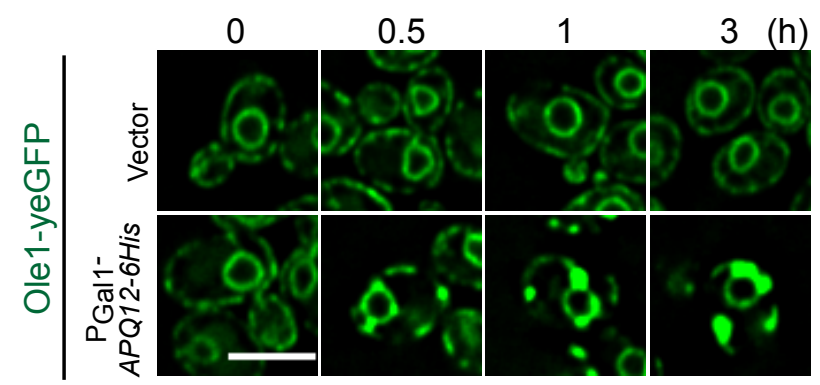




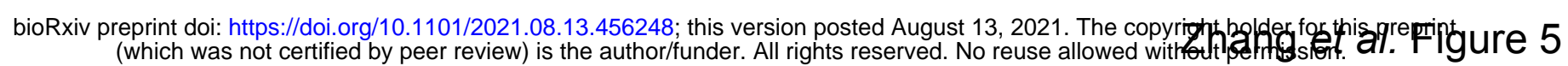

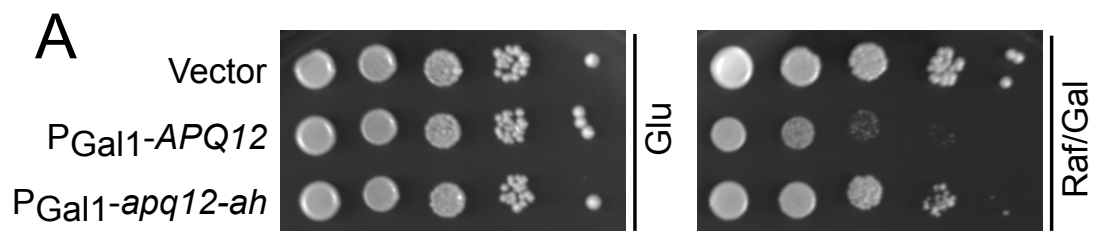

B

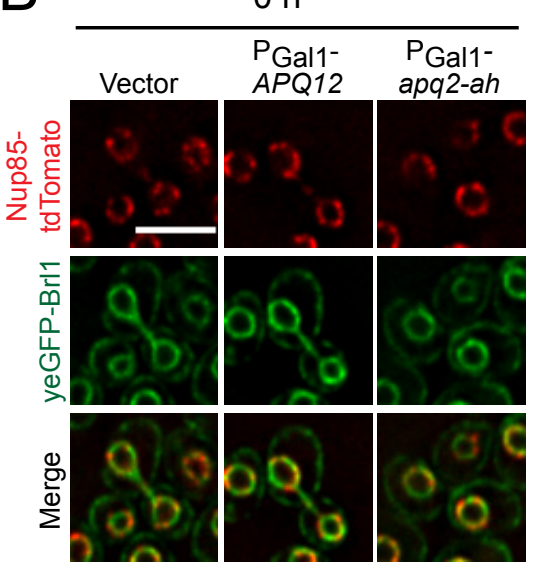

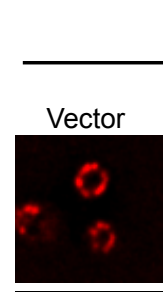
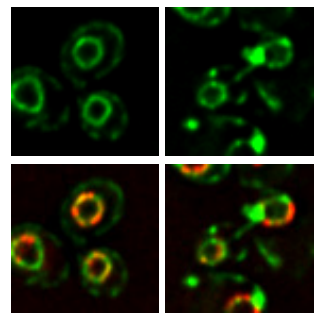

$1 \mathrm{~h}$

PGal1-
APQ12
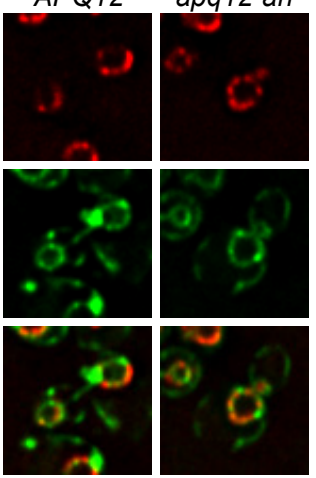

$3 \mathrm{~h}$

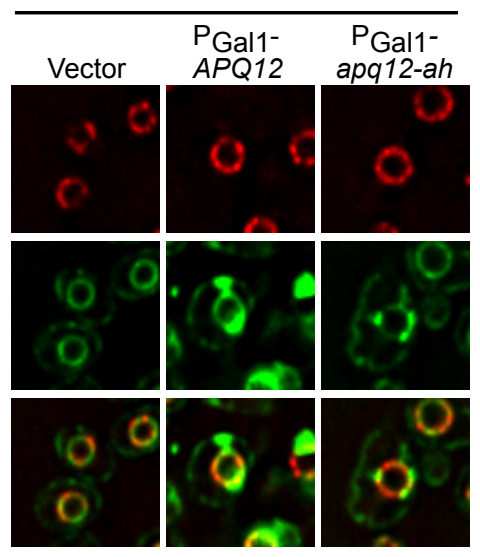

C

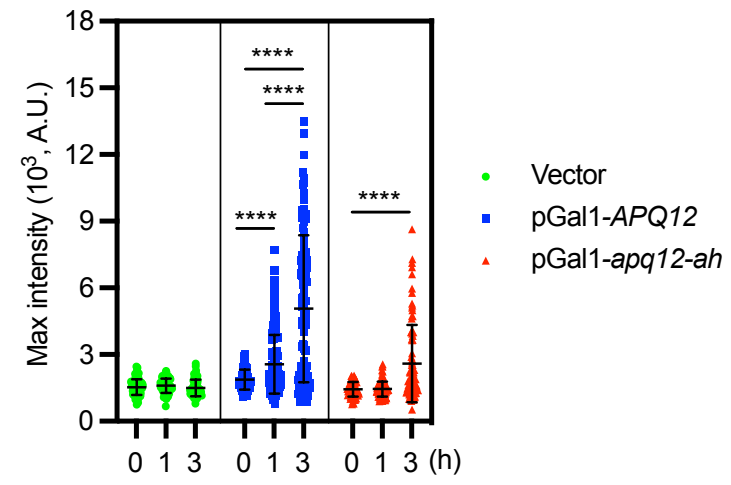

D
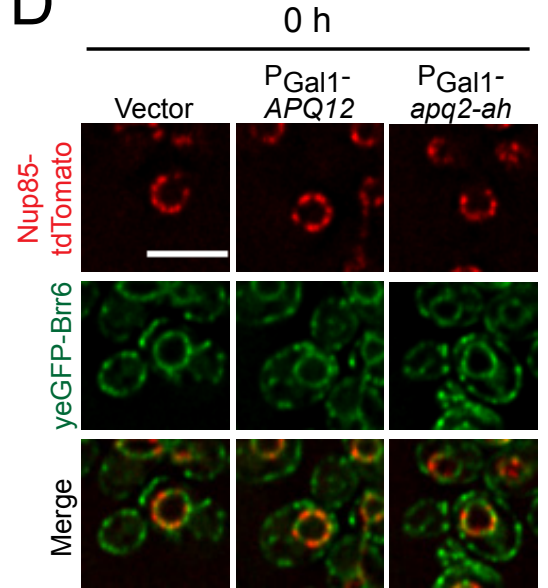
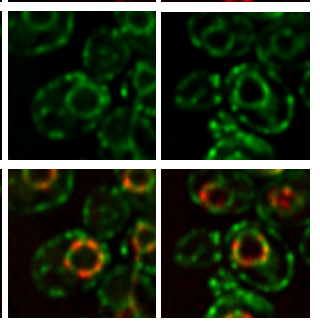
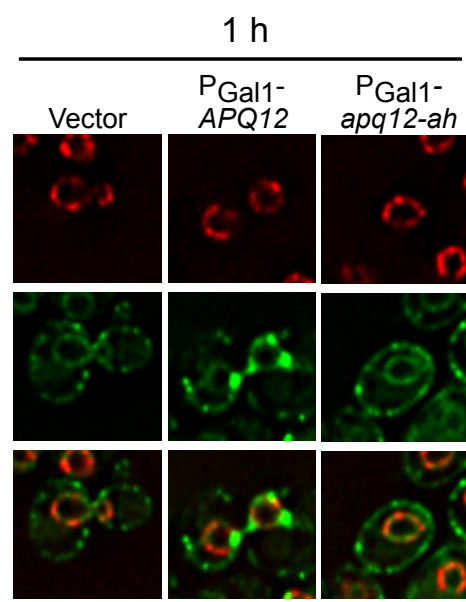
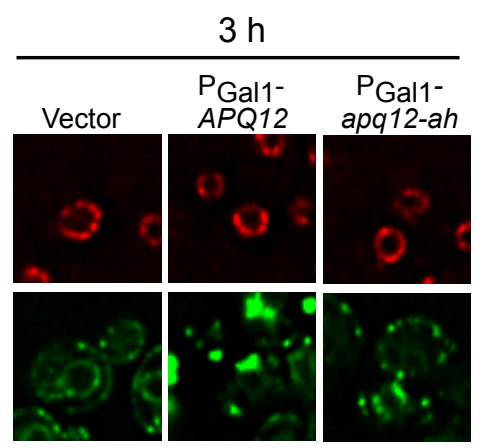

E

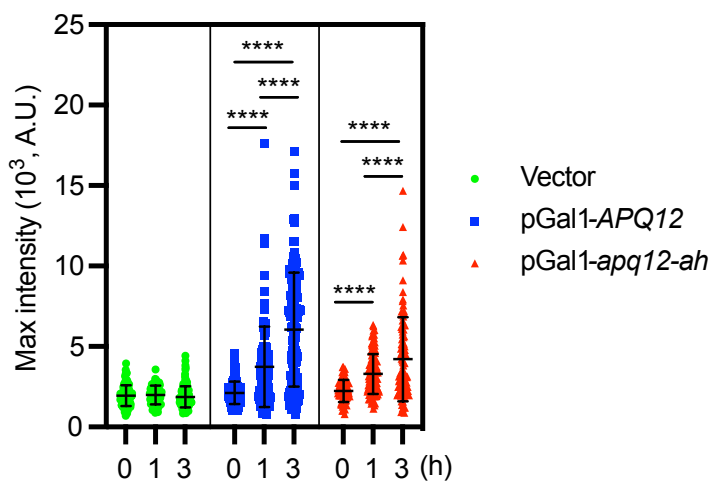


A

$$
\mathrm{P}_{\text {GAL1 }}-A P Q 12-6 H i s
$$
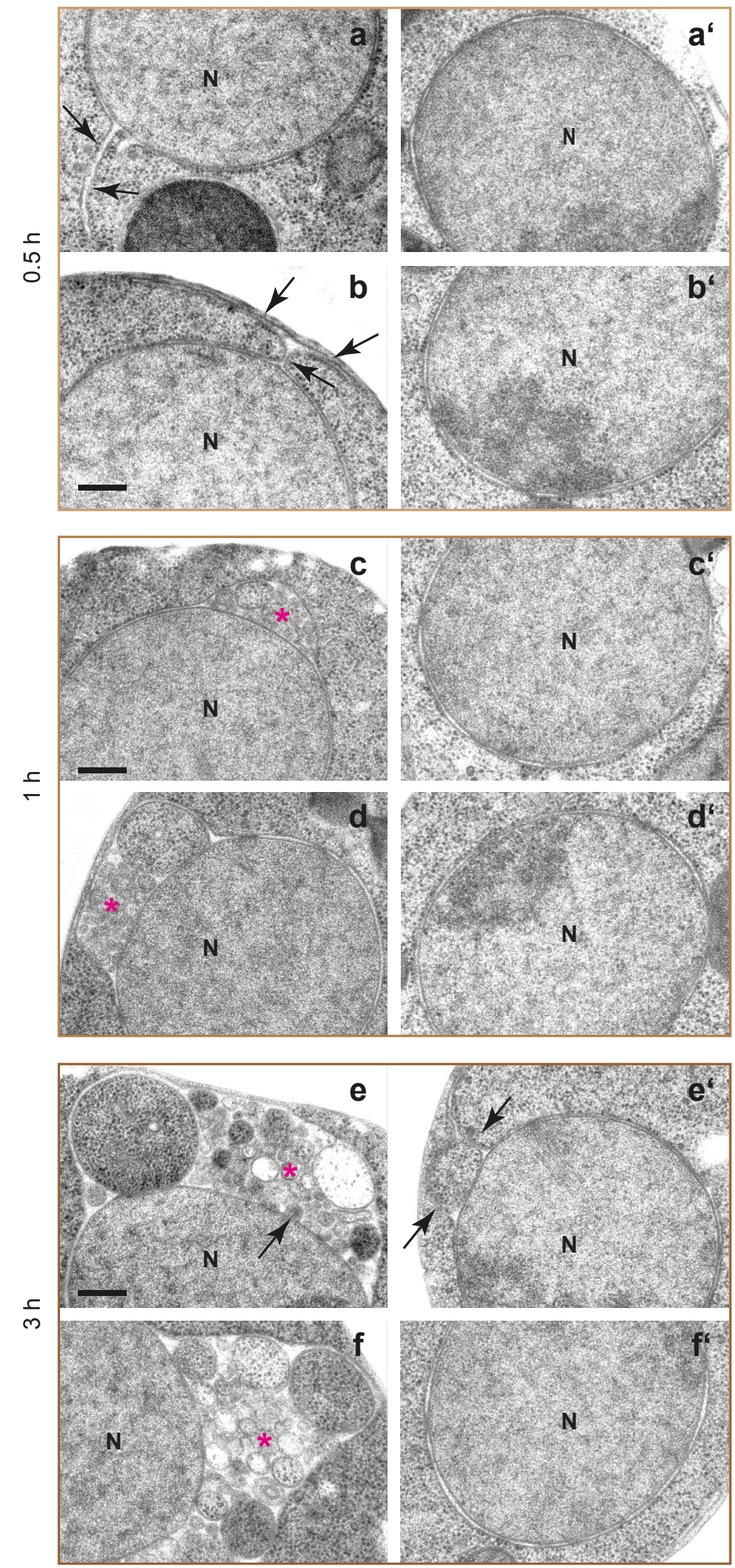

B

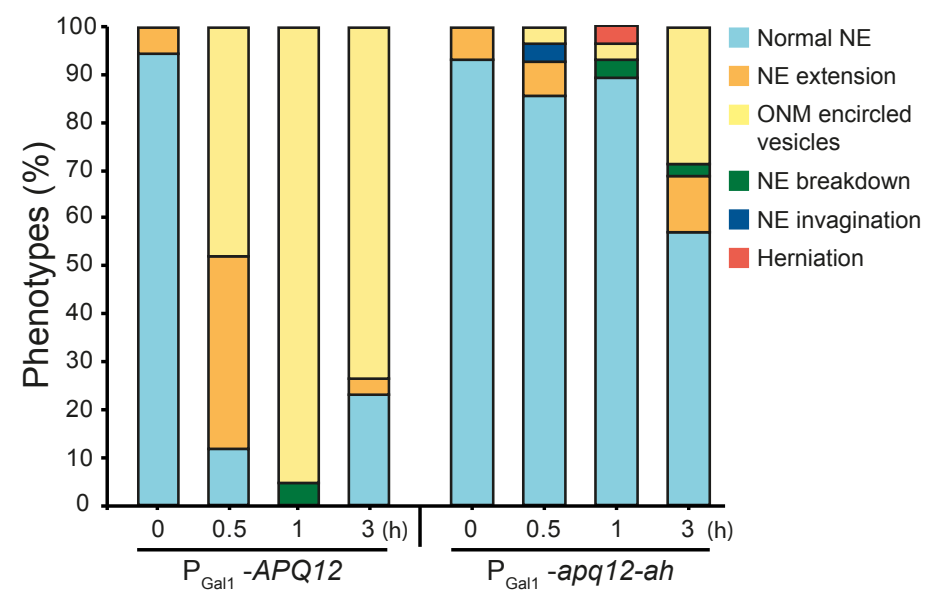

C
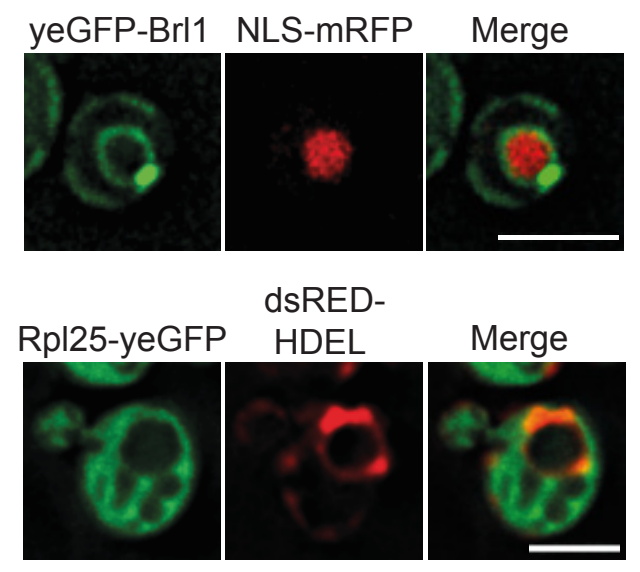

D

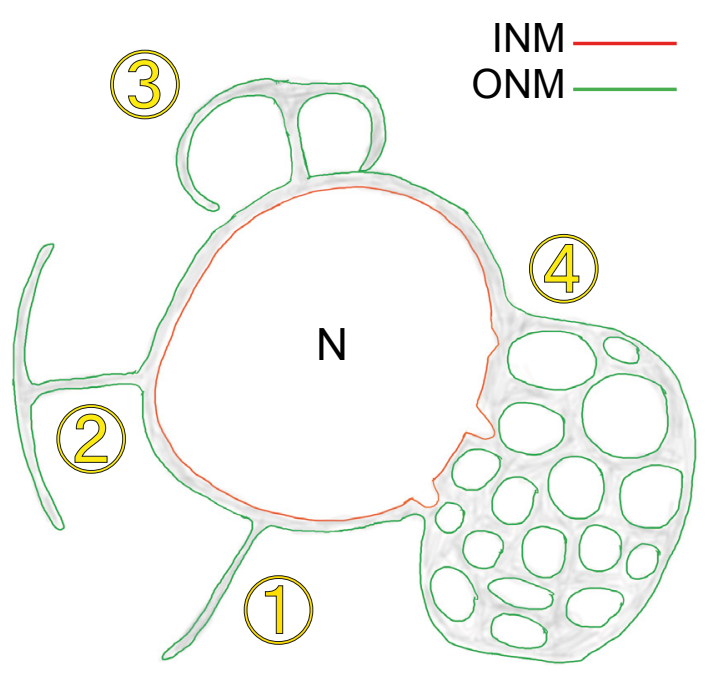


A
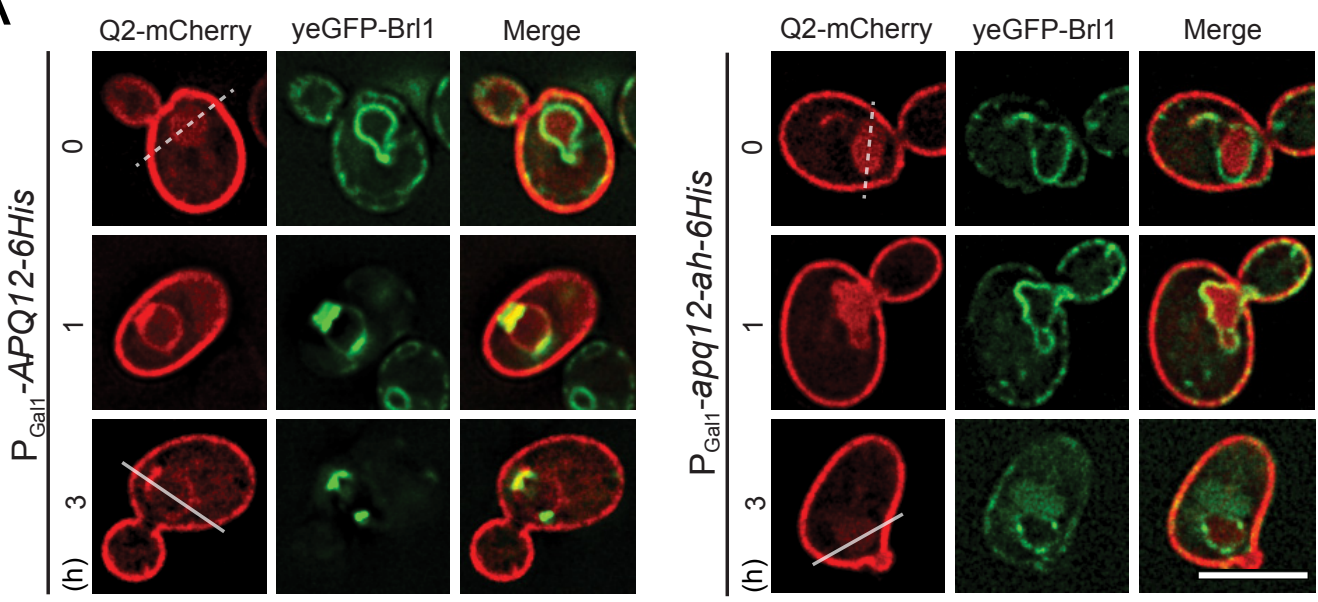

B
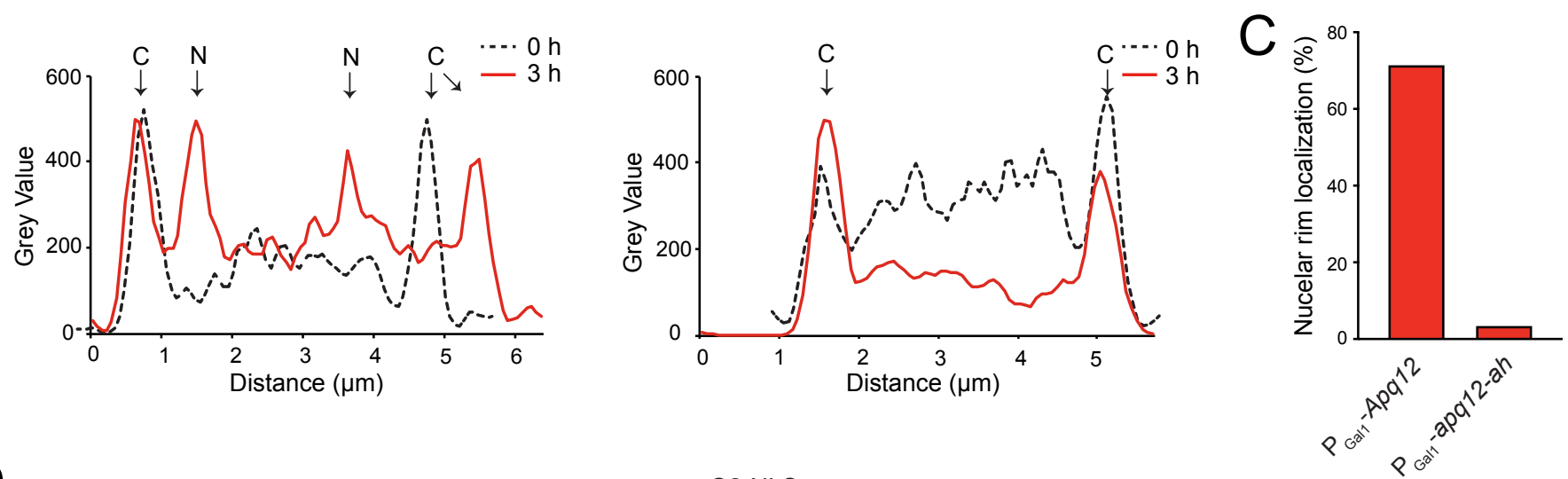

D
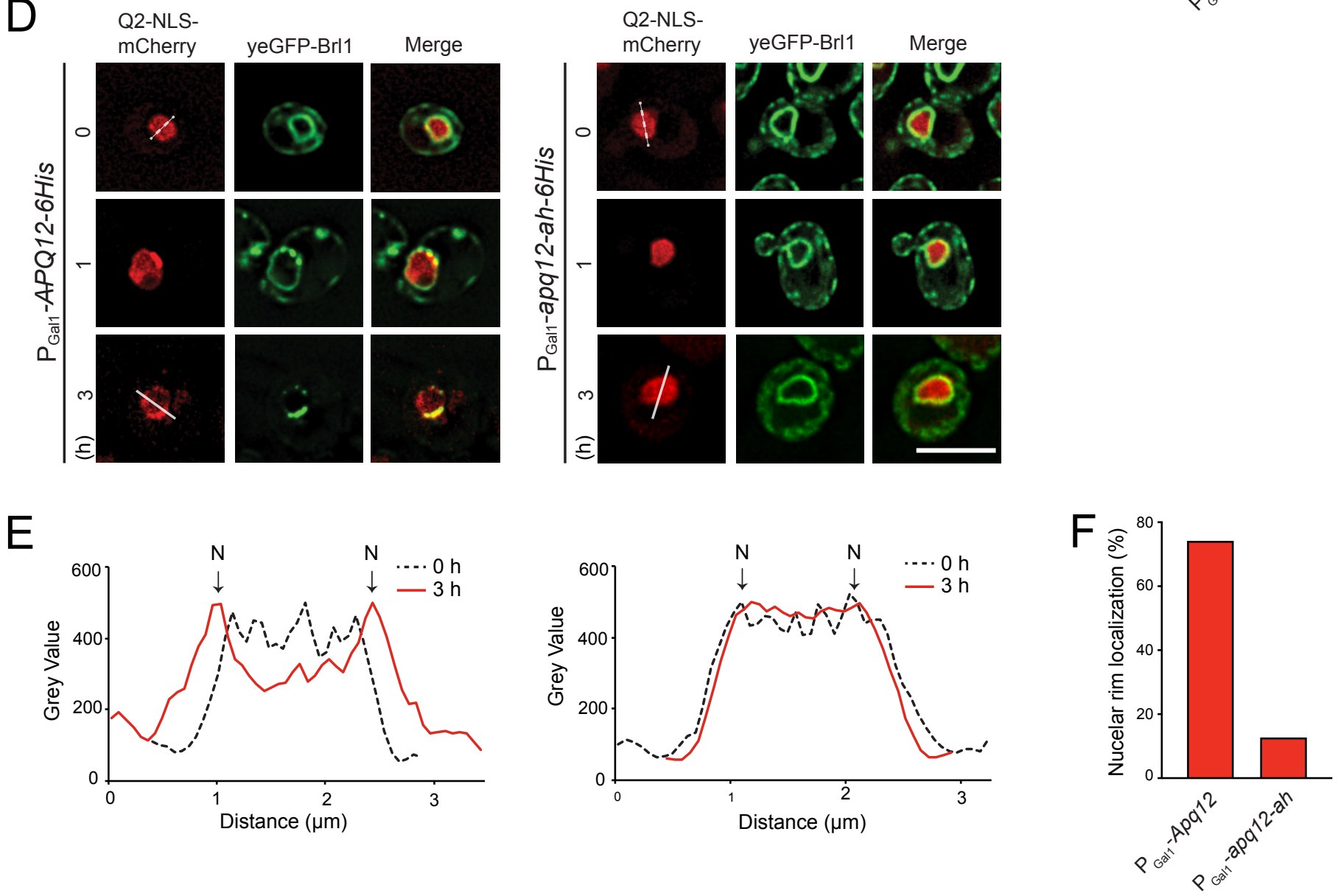


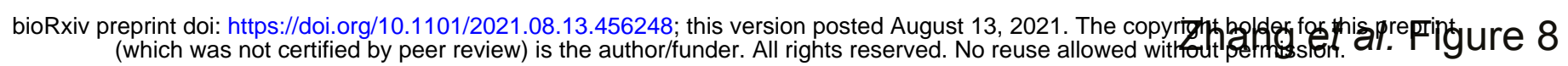

A
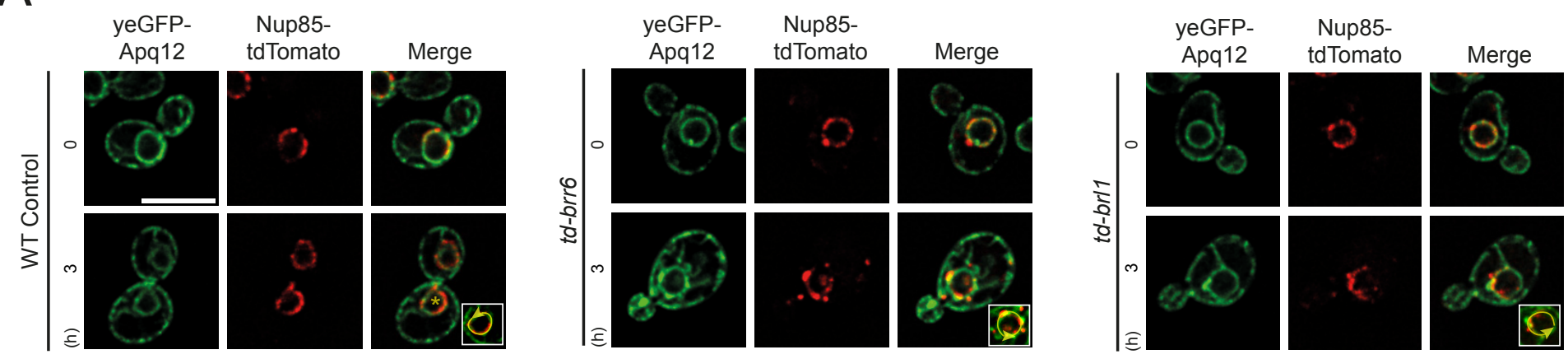

B
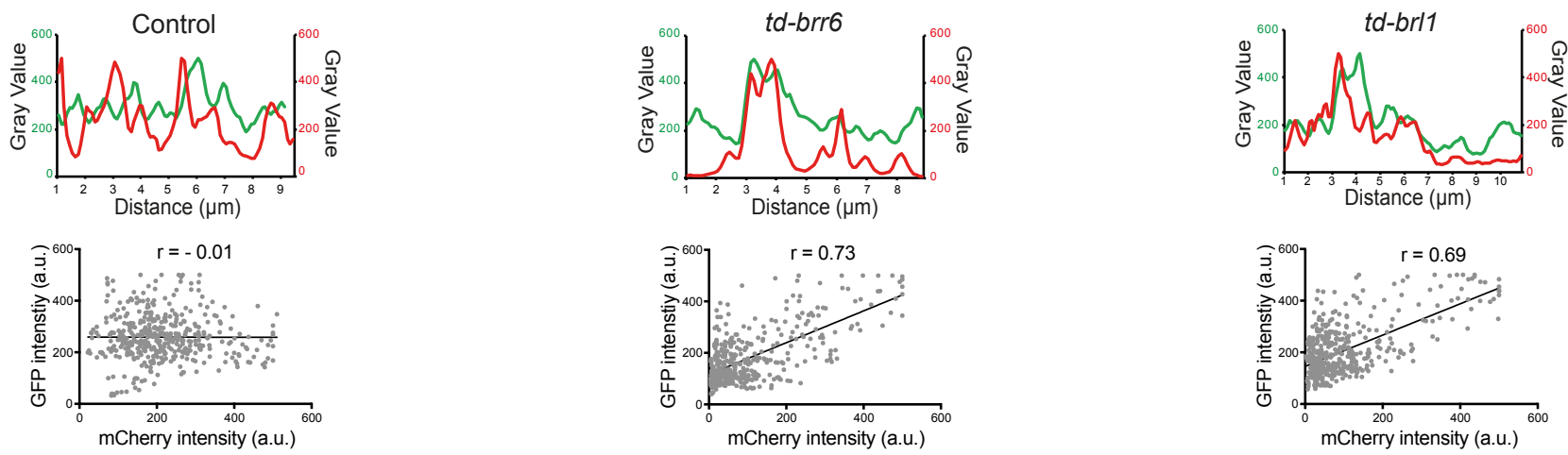

C

D
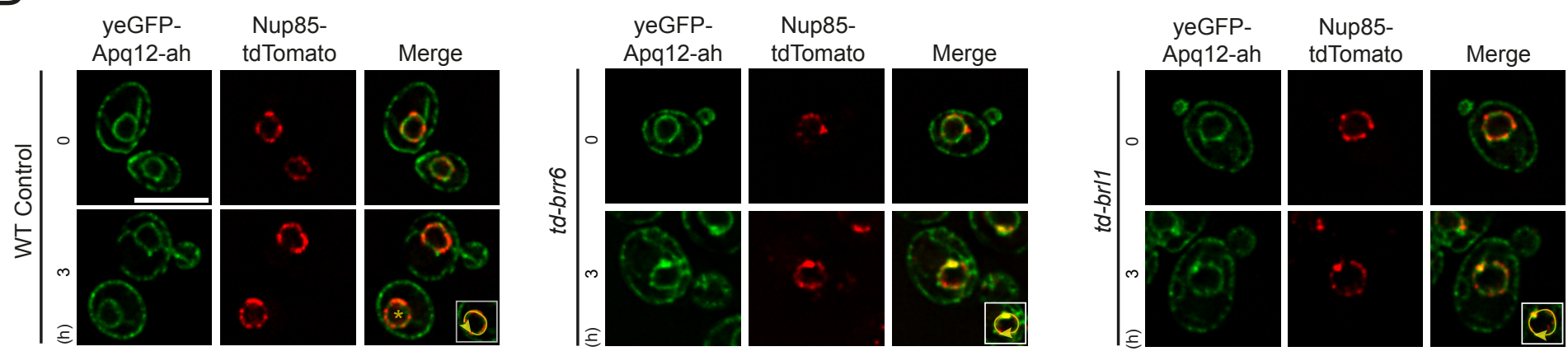

E
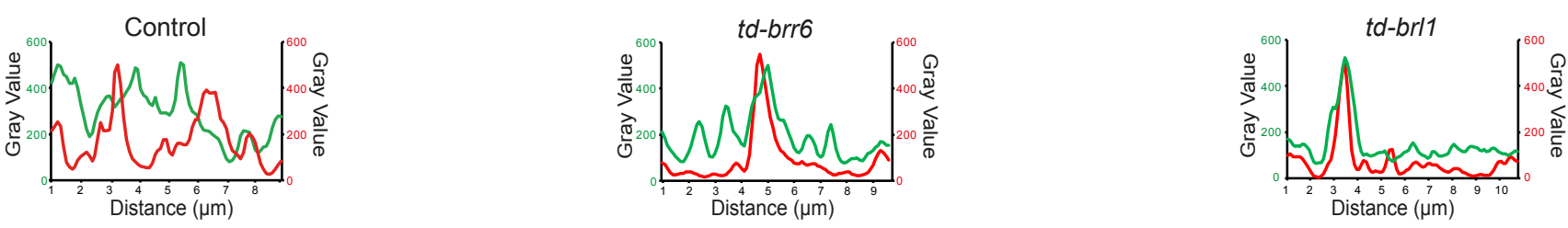

F
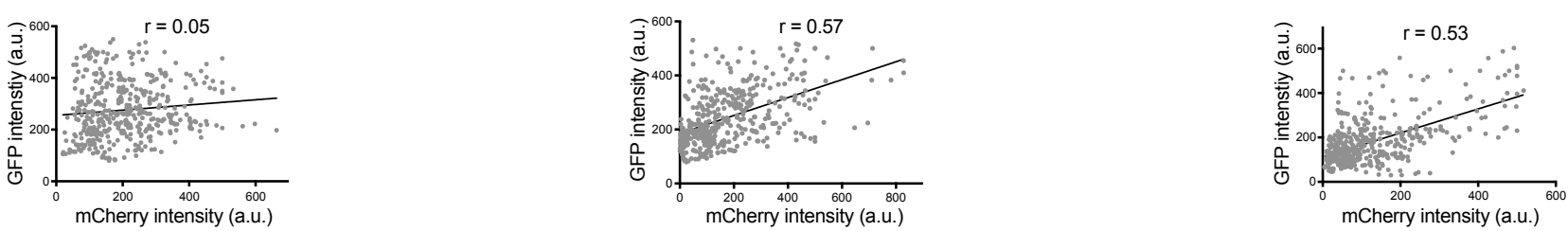

G

td-brl1 yeGFP-APQ12
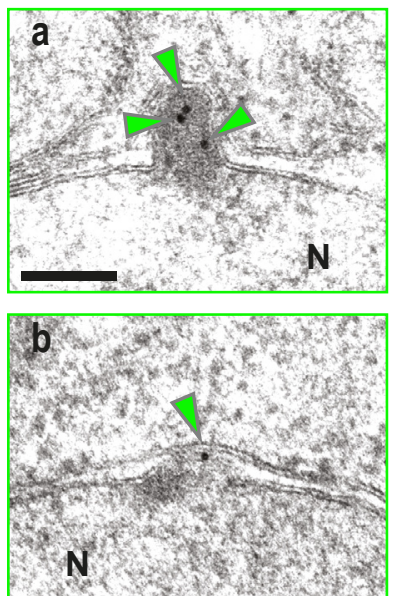

td-brl1 yeGFP-apq12-ah
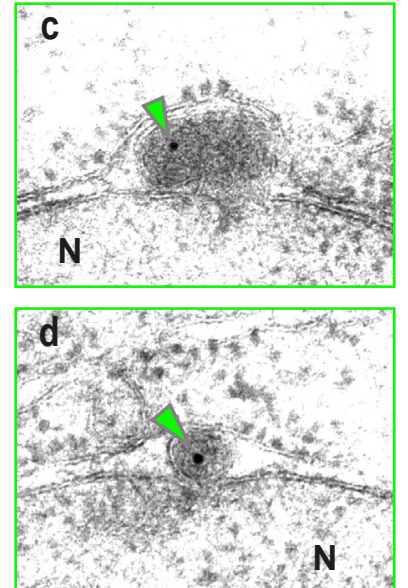

td-brr6 yeGFP-APQ12

td-brr6 yeGFP-apq12-ah
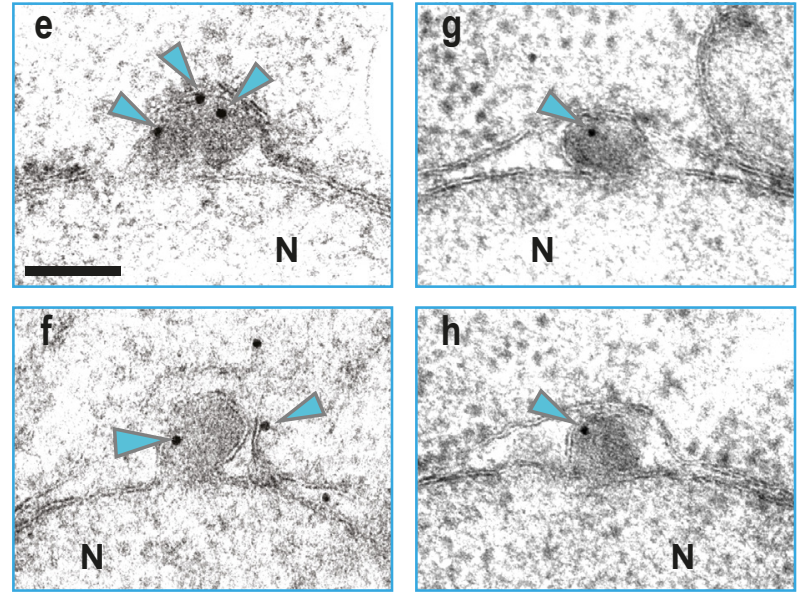
A
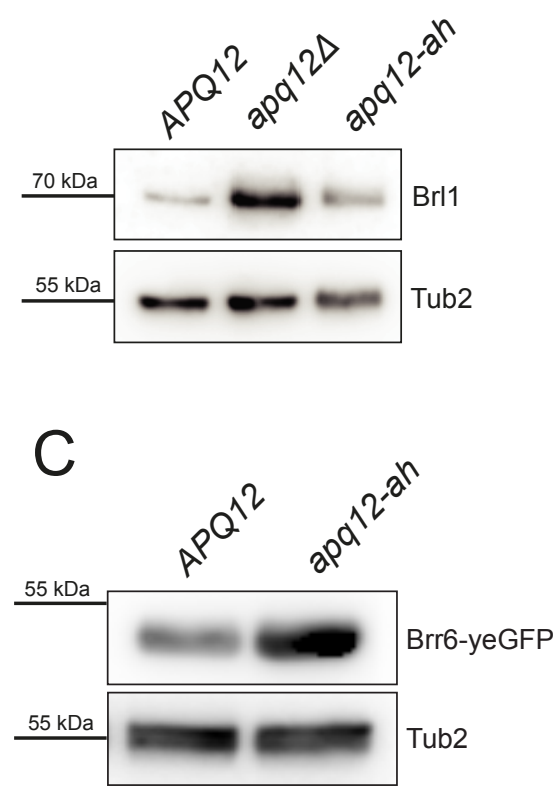

E
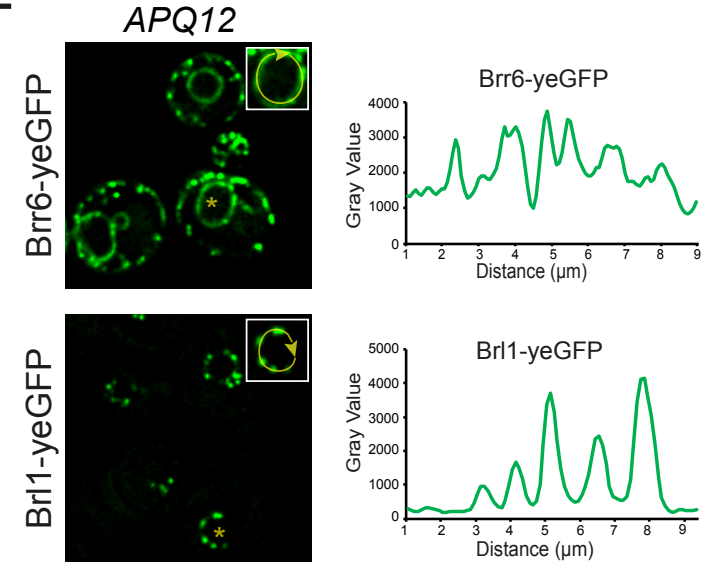

B

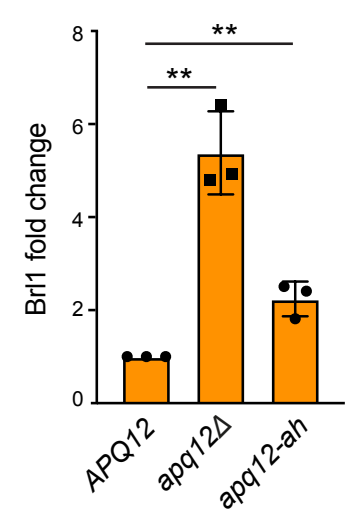

D

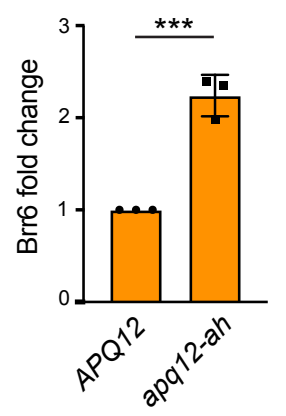

$\mathrm{F}$

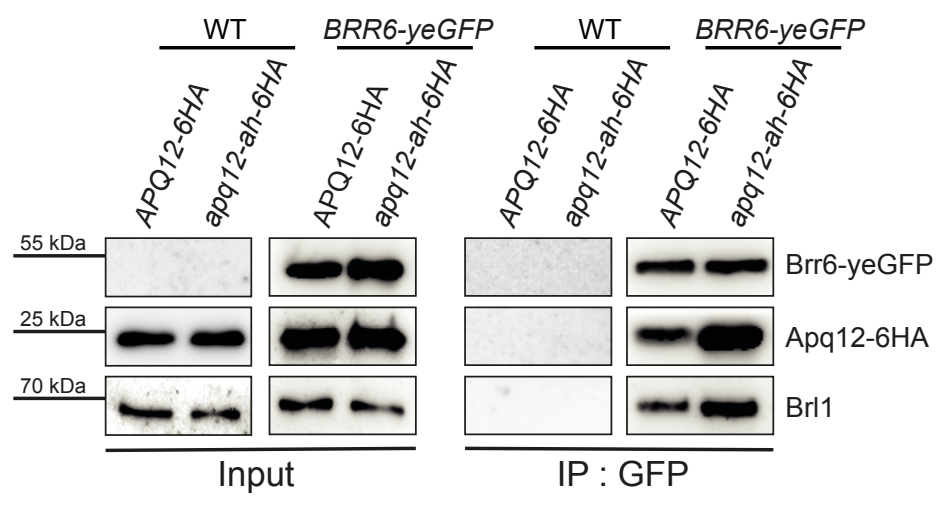

apq12-ah
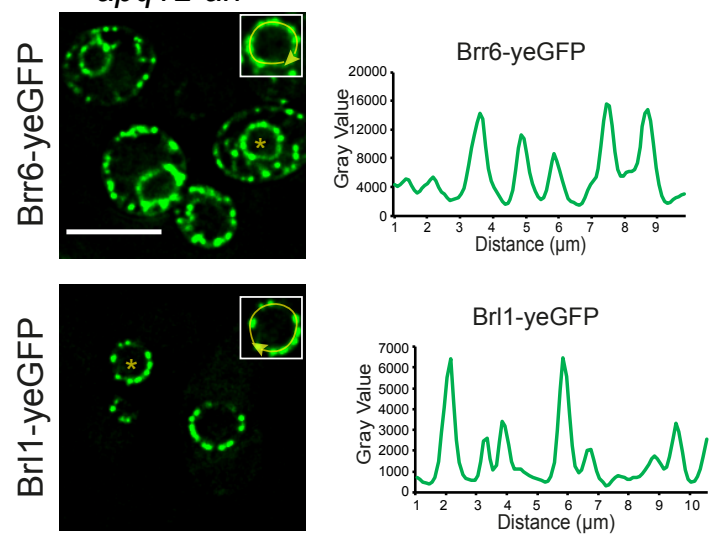

G
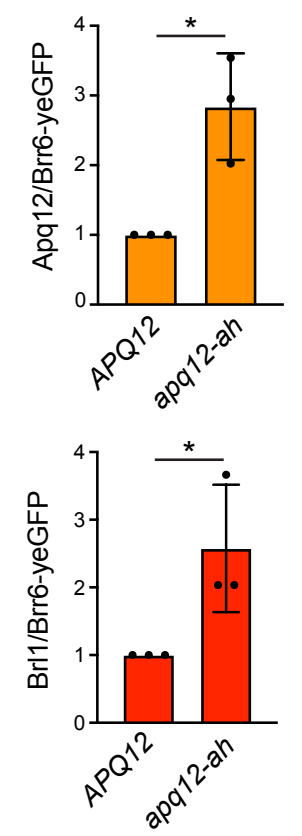
A

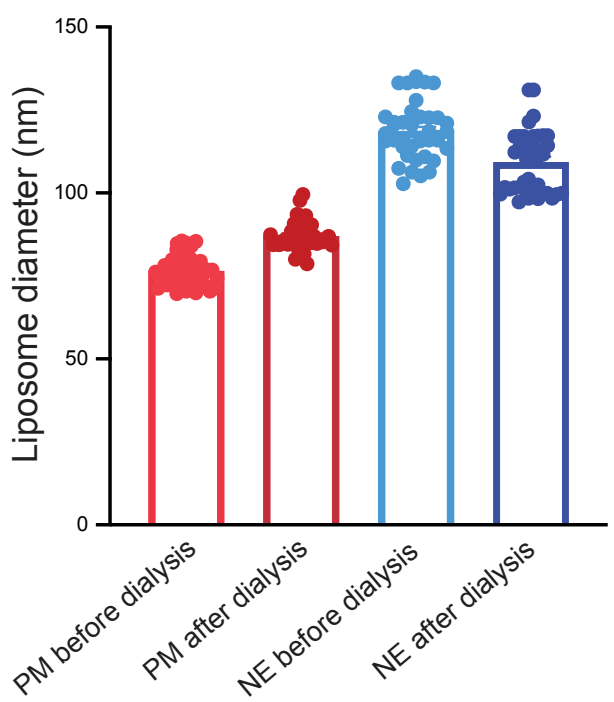

B

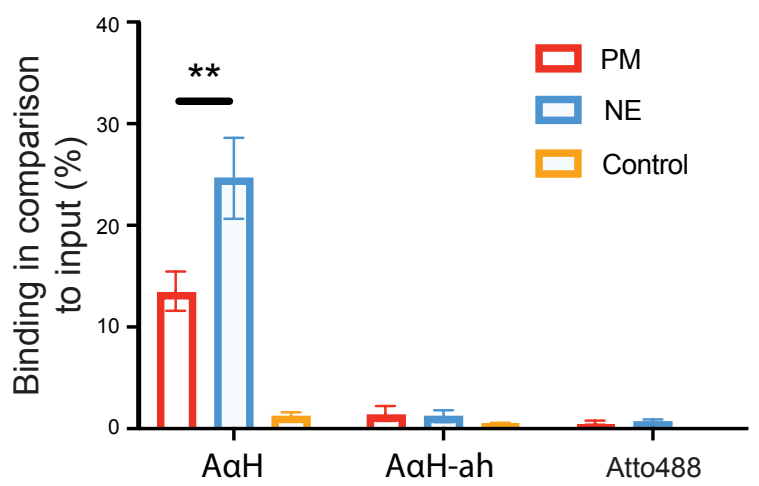

C

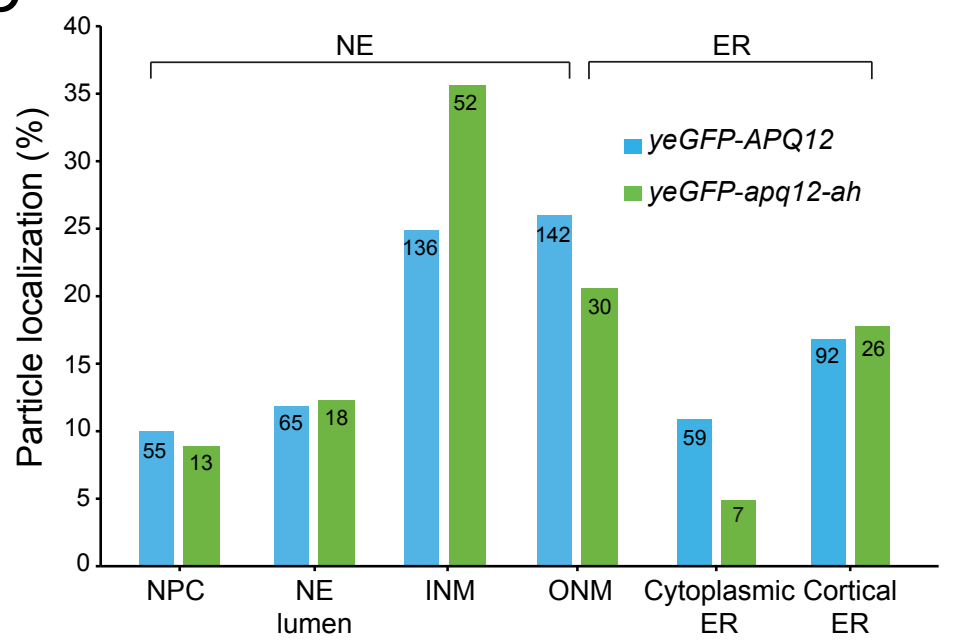

yeGFP-Apq12 marked NPCs $(10 \%)$

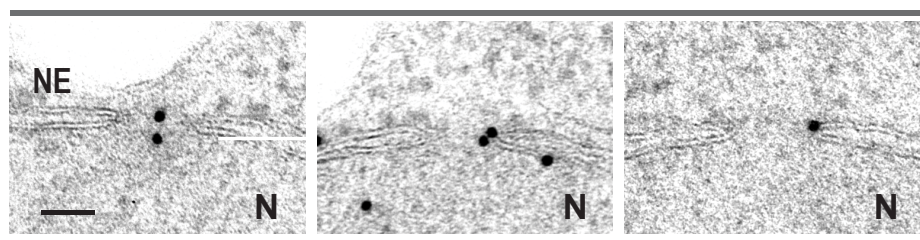

yeGFP-Apq12-ah marked NPCs (16\%)

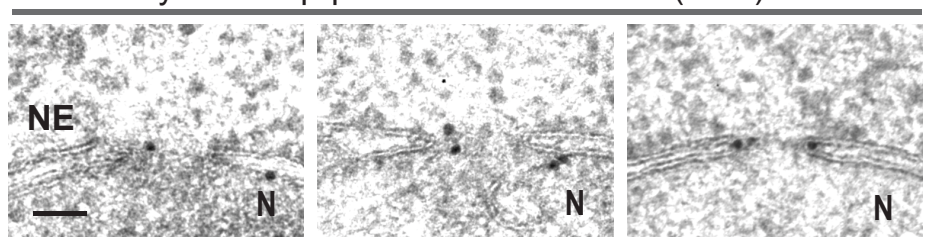

NPCs without yeGFP-Apq12 (90\%)

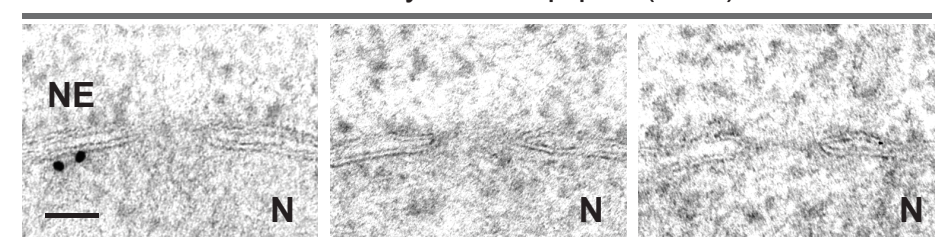

NPCs without yeGFP-Apq12-ah (84\%)

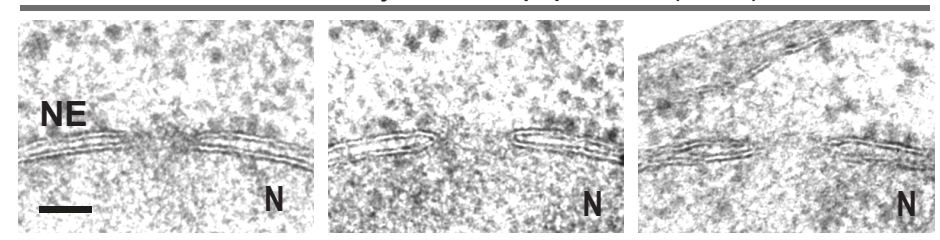

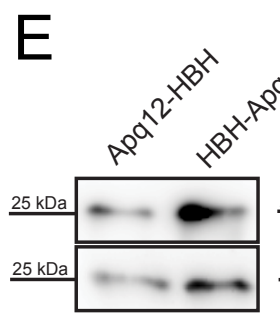
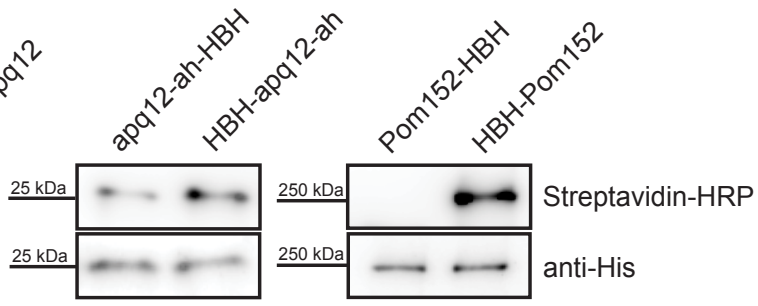
A
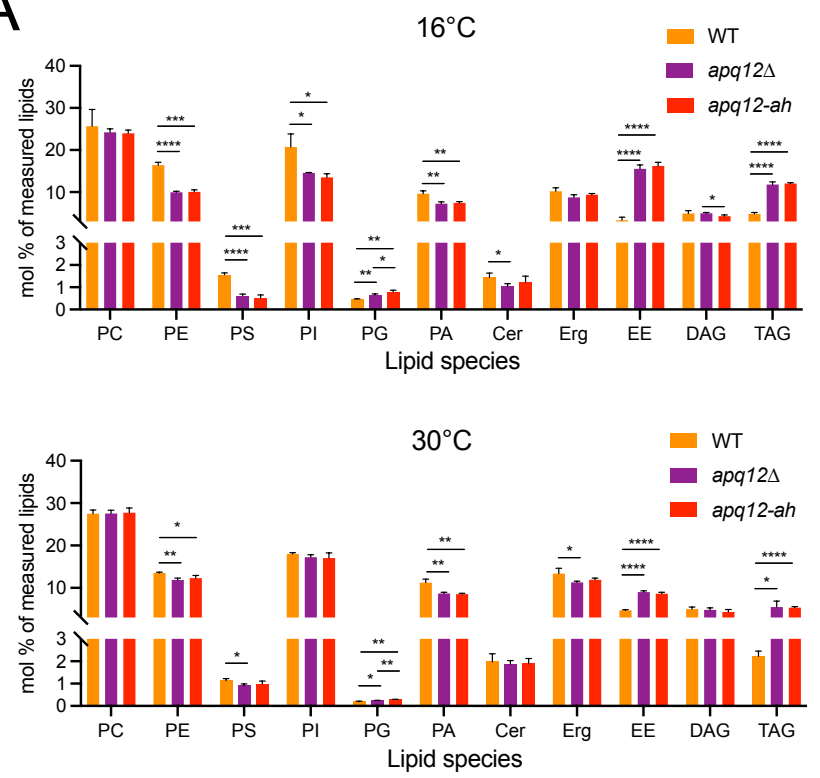

$37^{\circ} \mathrm{C}$

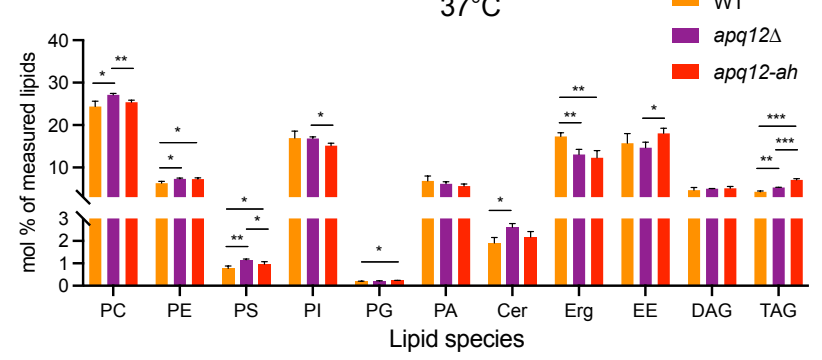

B
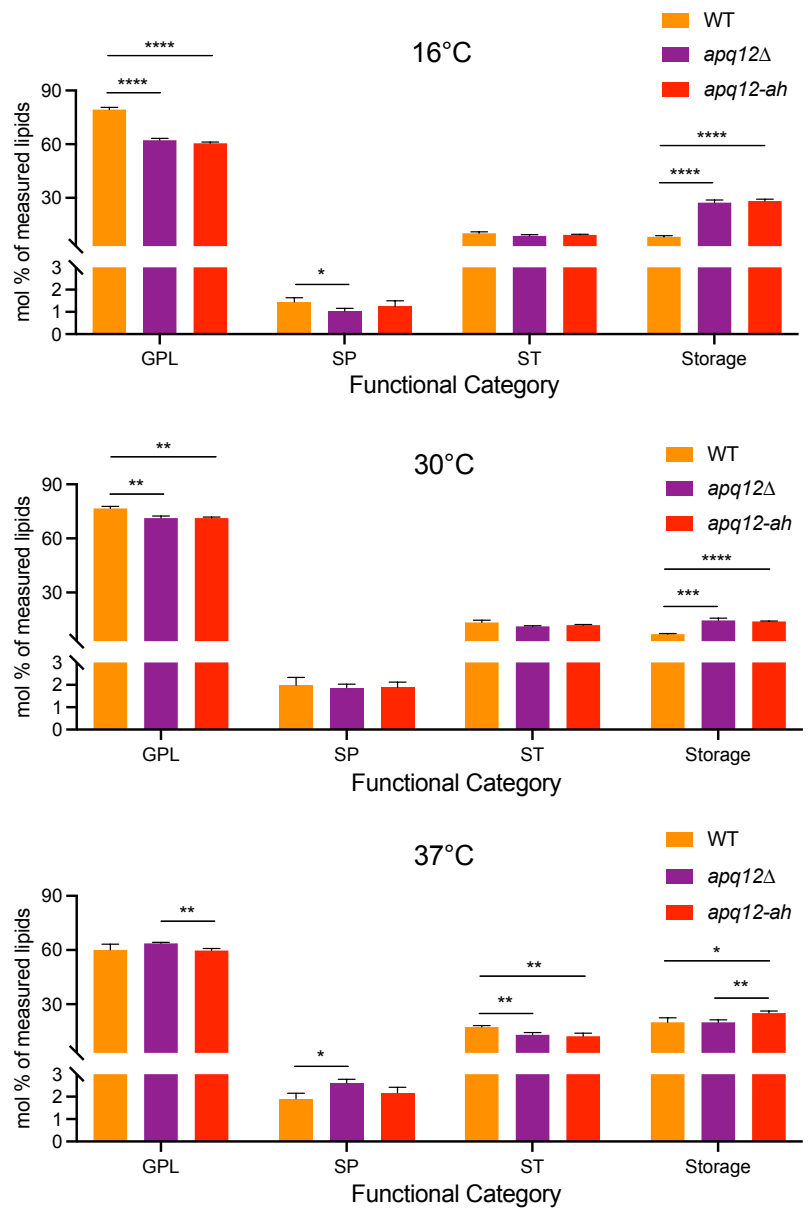

C
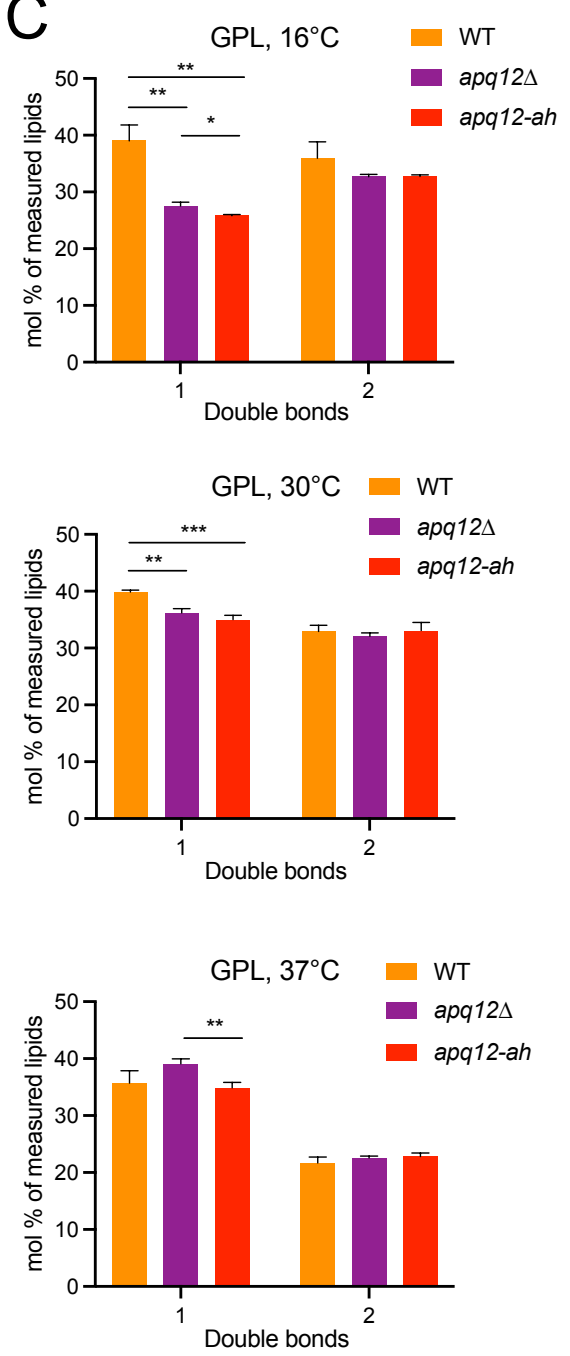

D

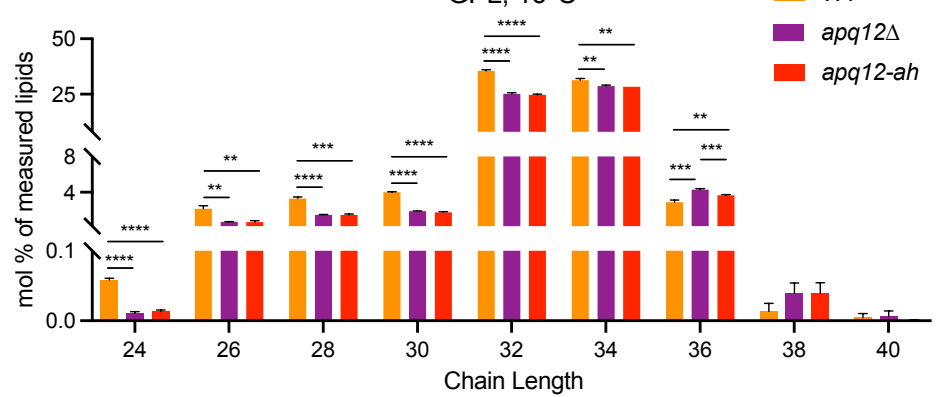

$\mathrm{GPL}, 30^{\circ} \mathrm{C}$

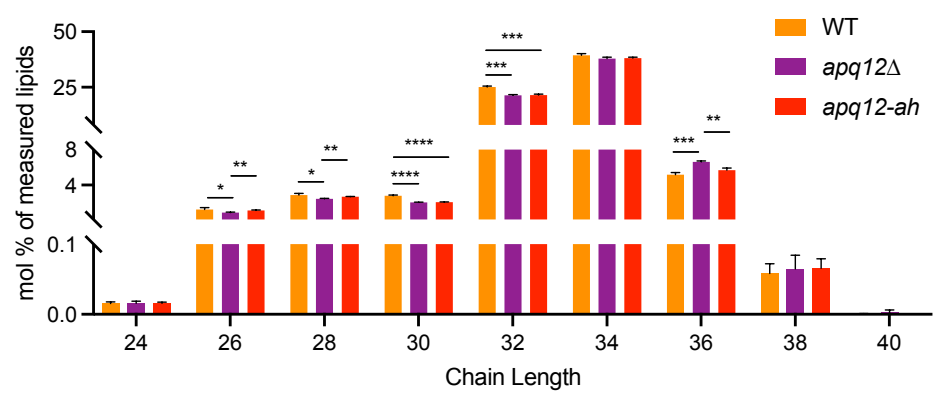

$\mathrm{GPL}, 37^{\circ} \mathrm{C} \quad \mathrm{WT}$

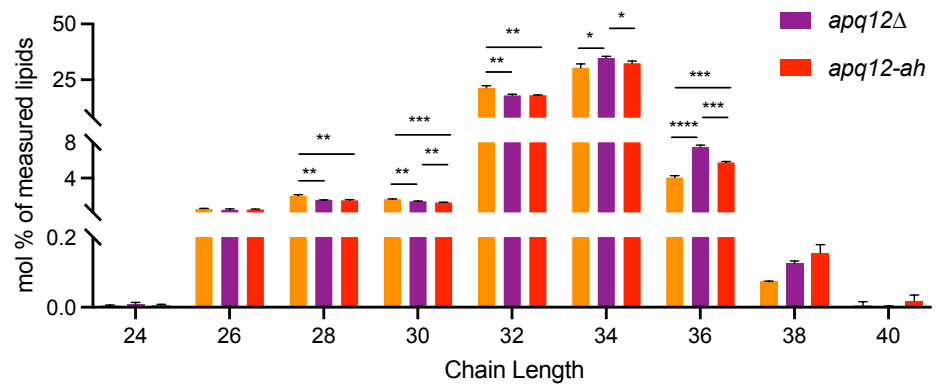


A

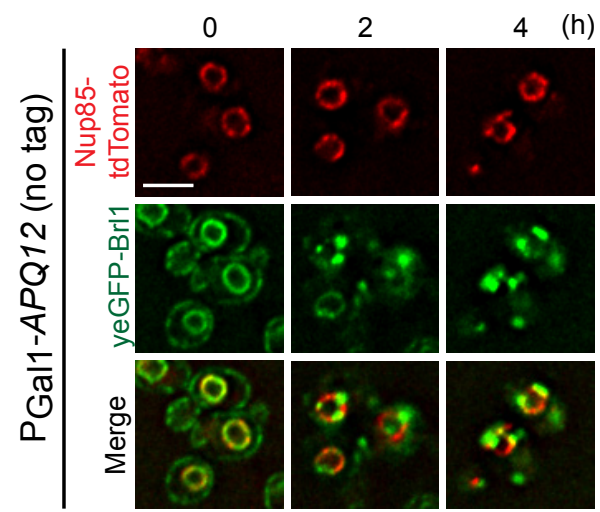

C

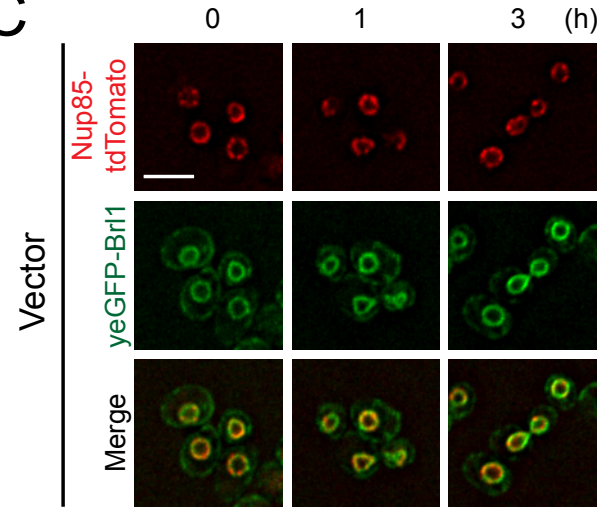

B

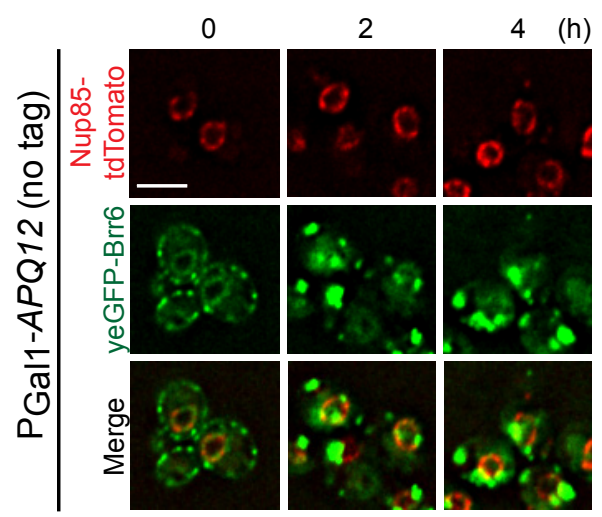

D

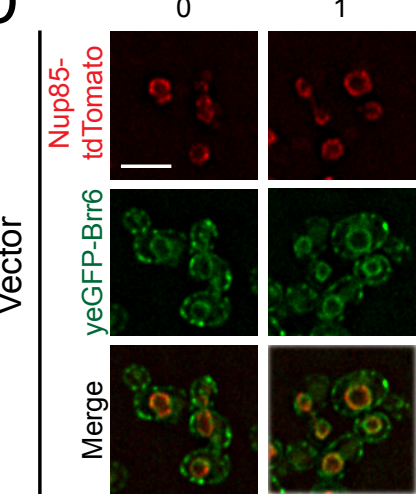

3 (h)
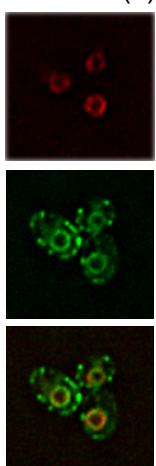

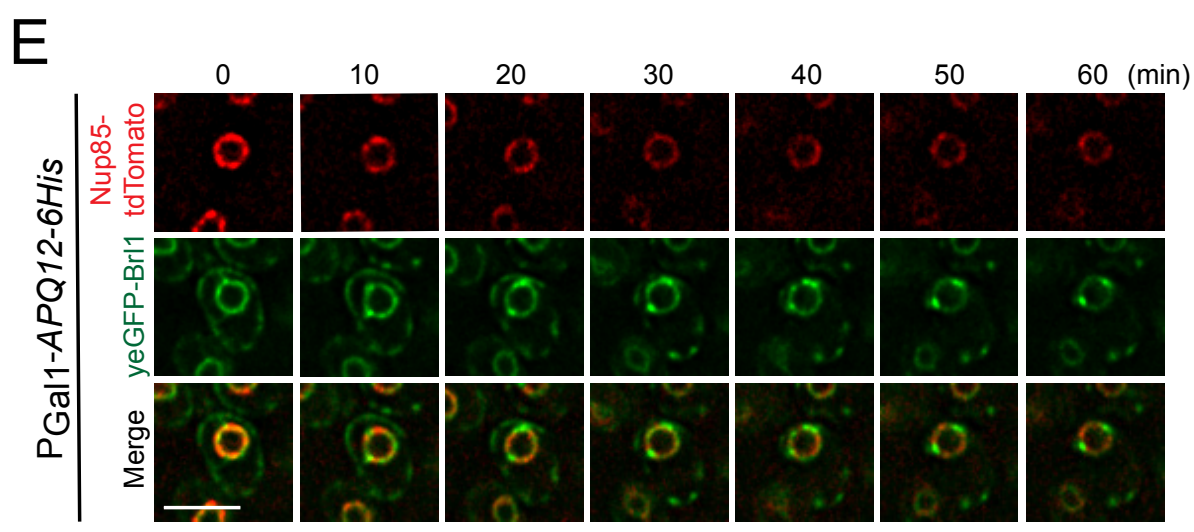

F

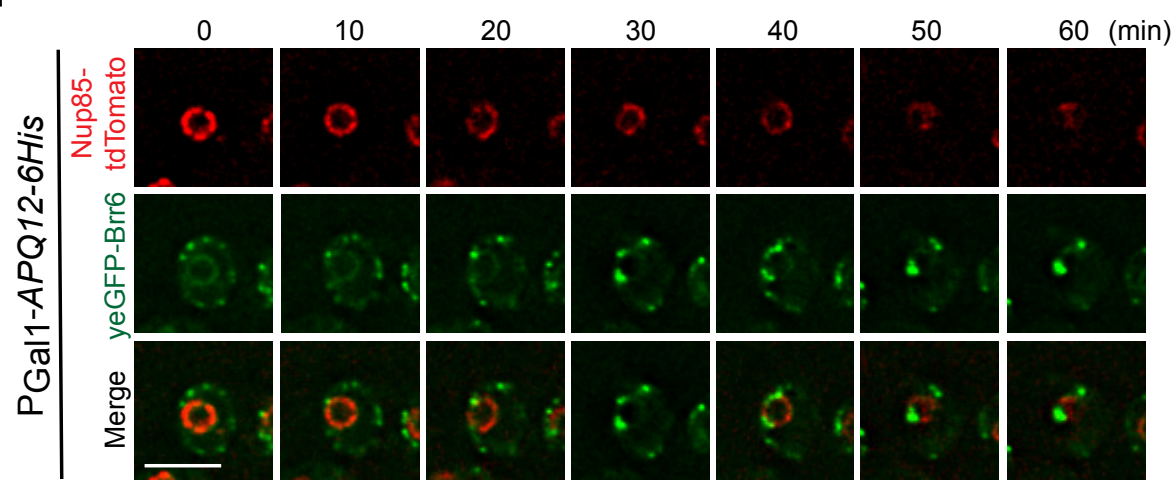



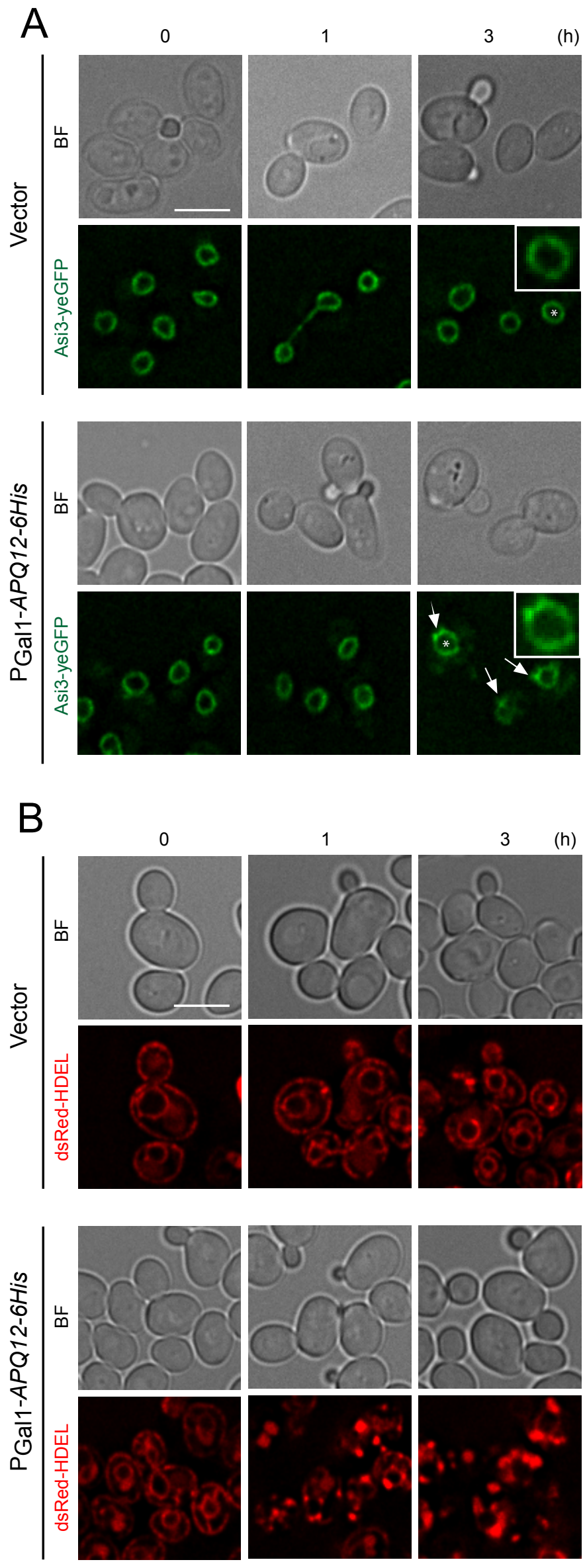
A

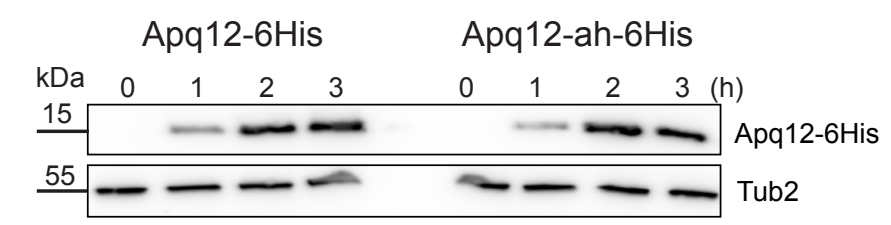

B
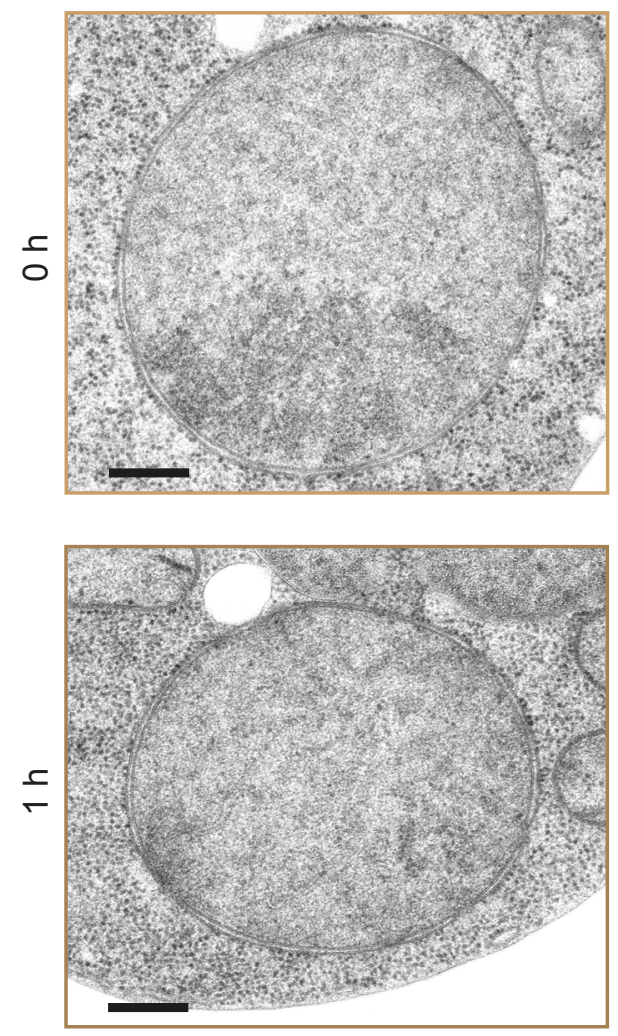

C PGa11-APQ12-yeGFP

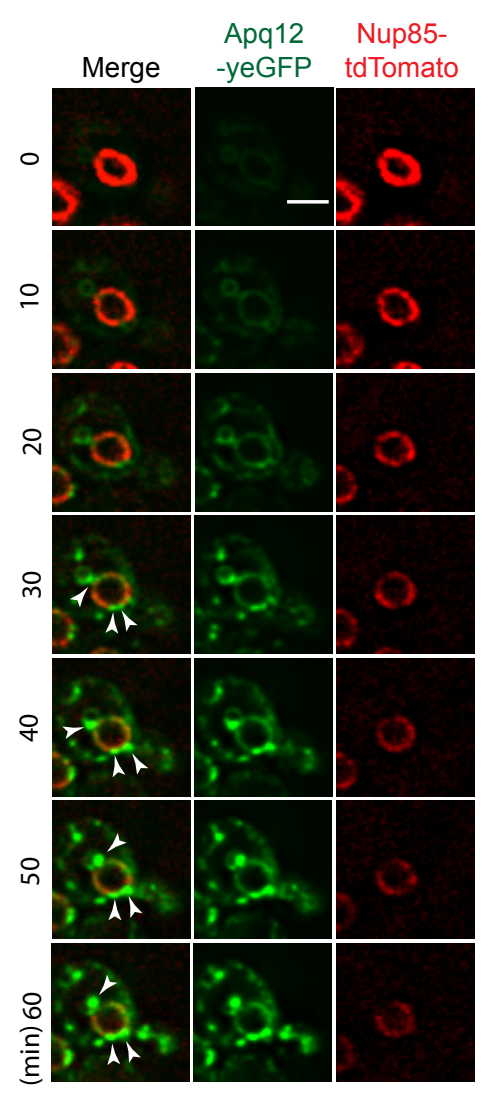

PGal1-apq12-ah-yeGFP

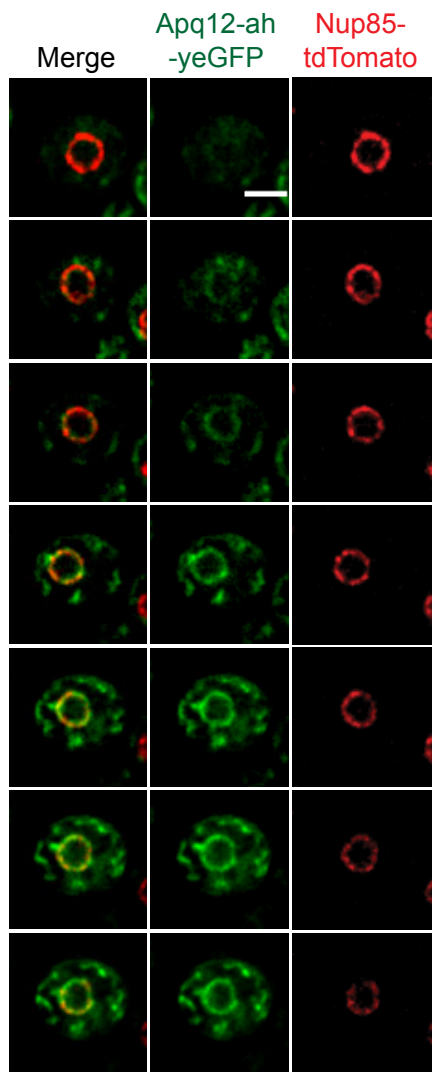

D

PGal1-APQ12-yeGFP
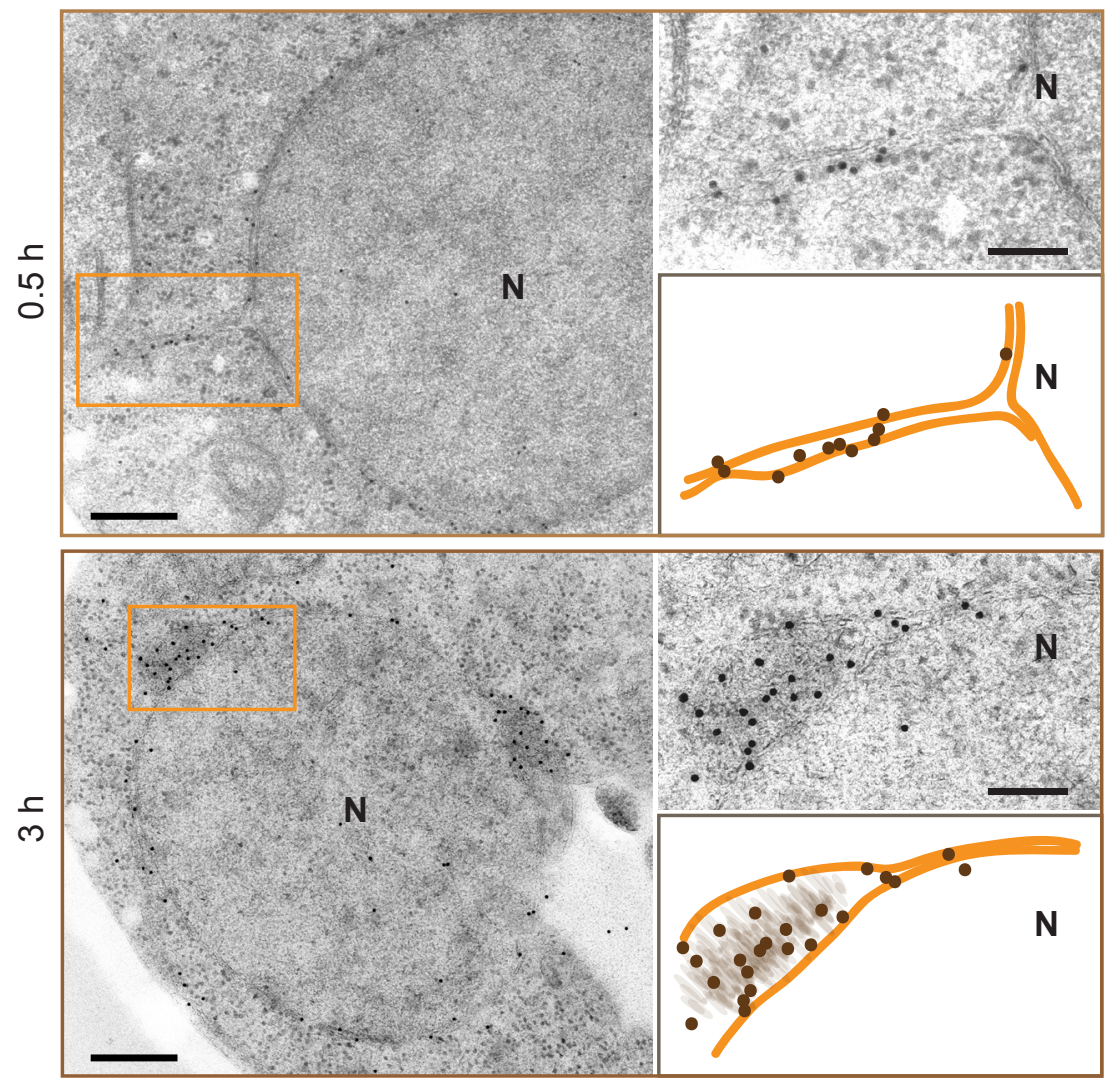
A
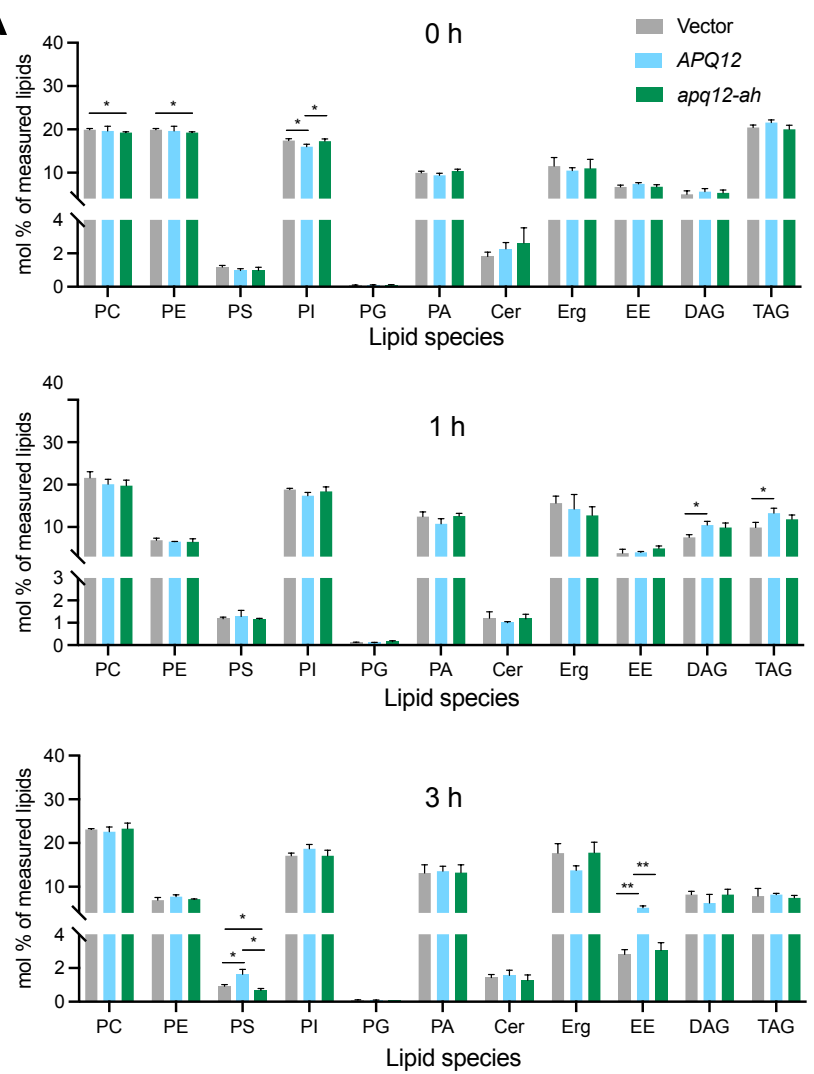

B
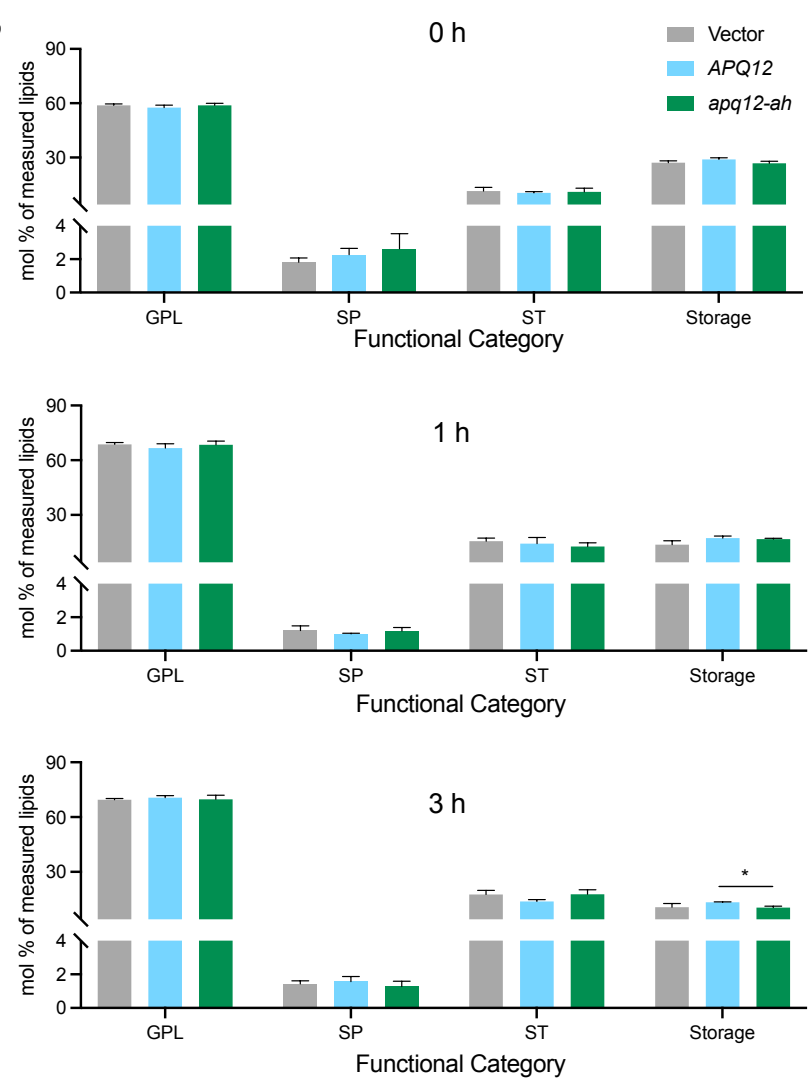

C
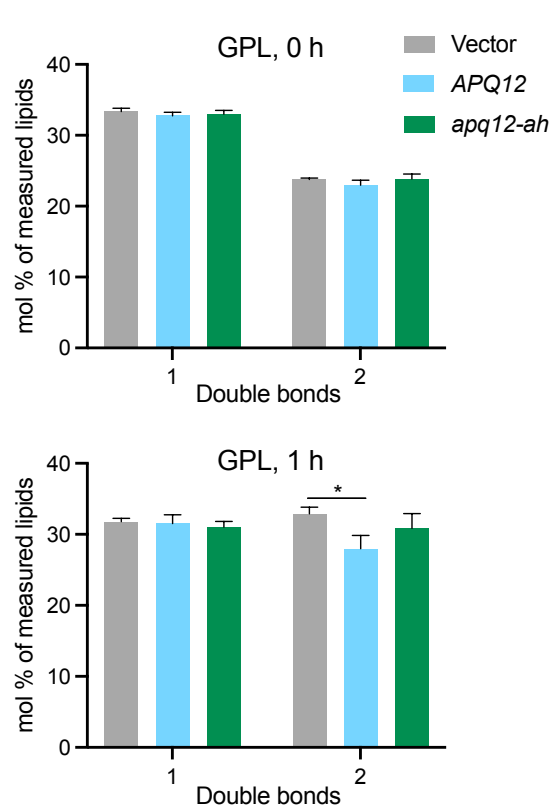

GPL, $3 \mathrm{~h}$

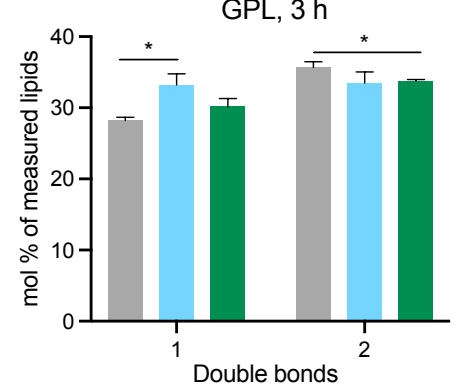

D
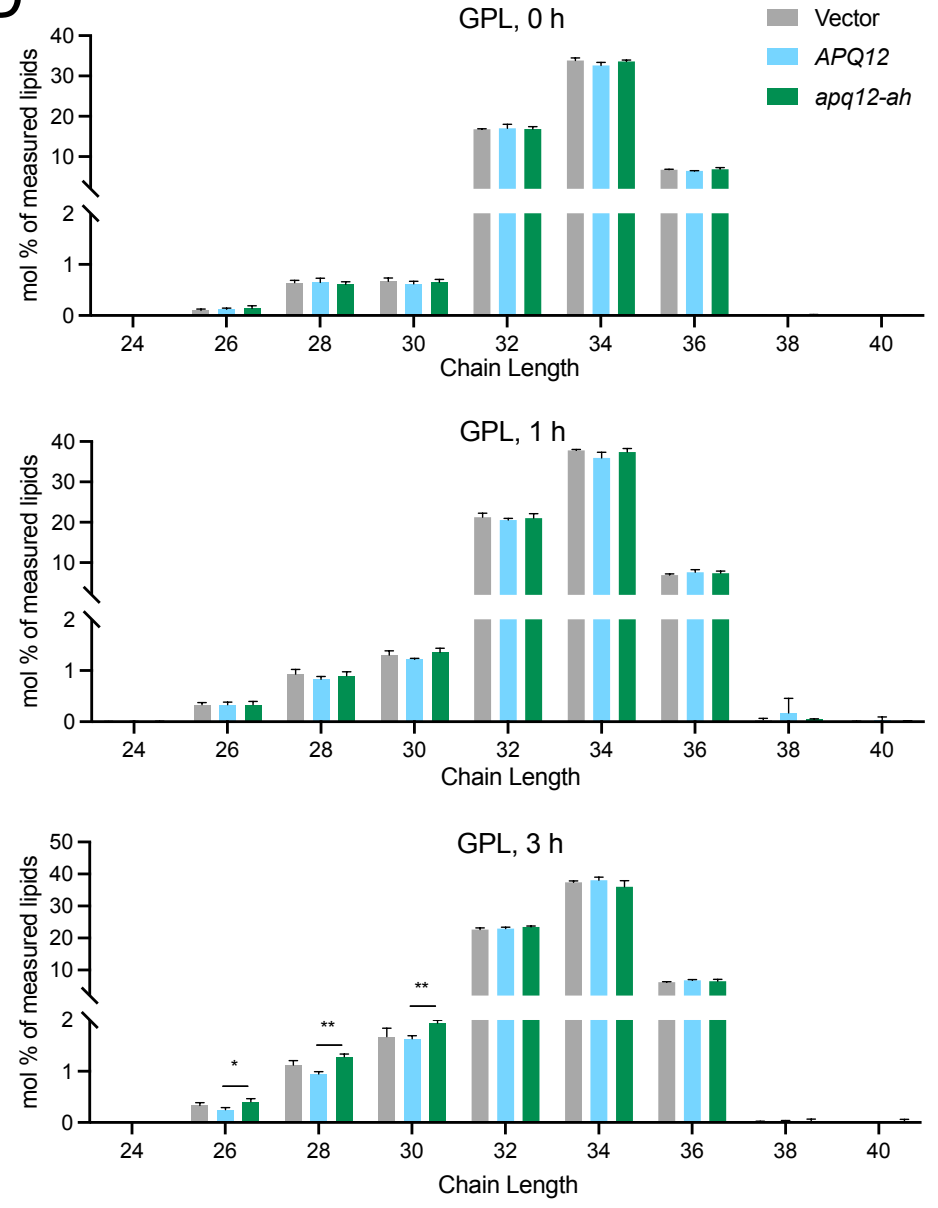
A
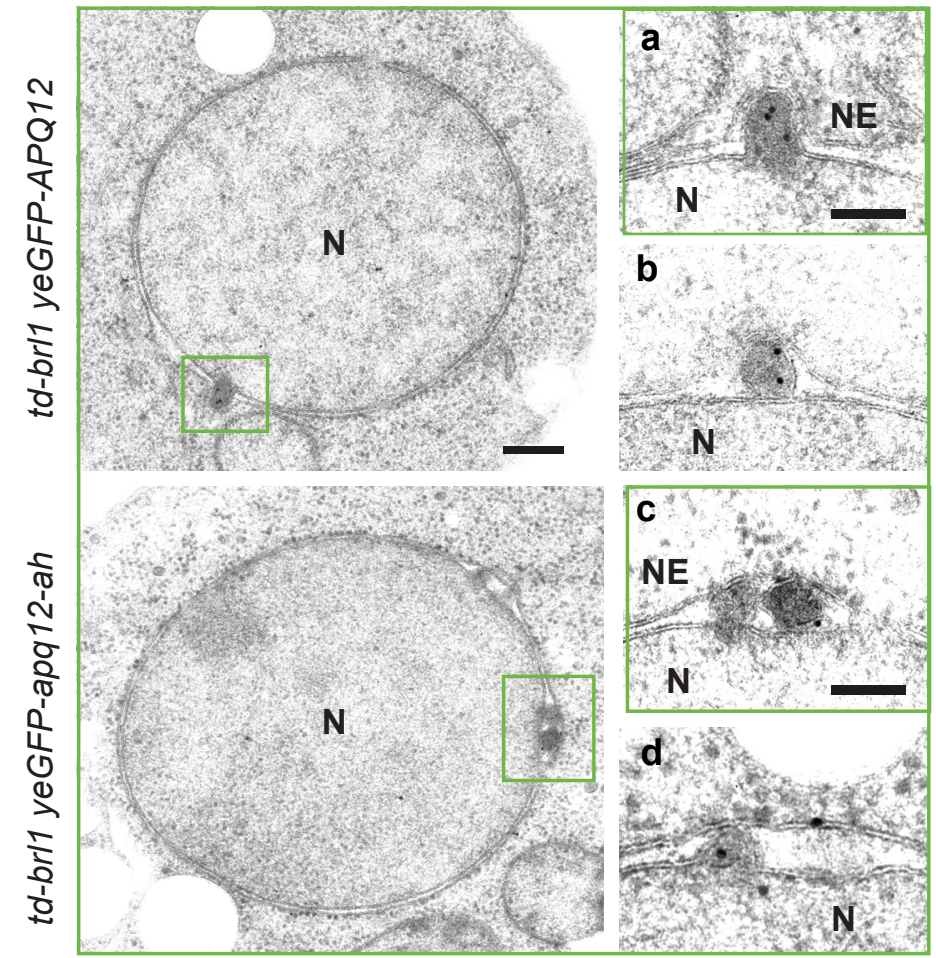

.

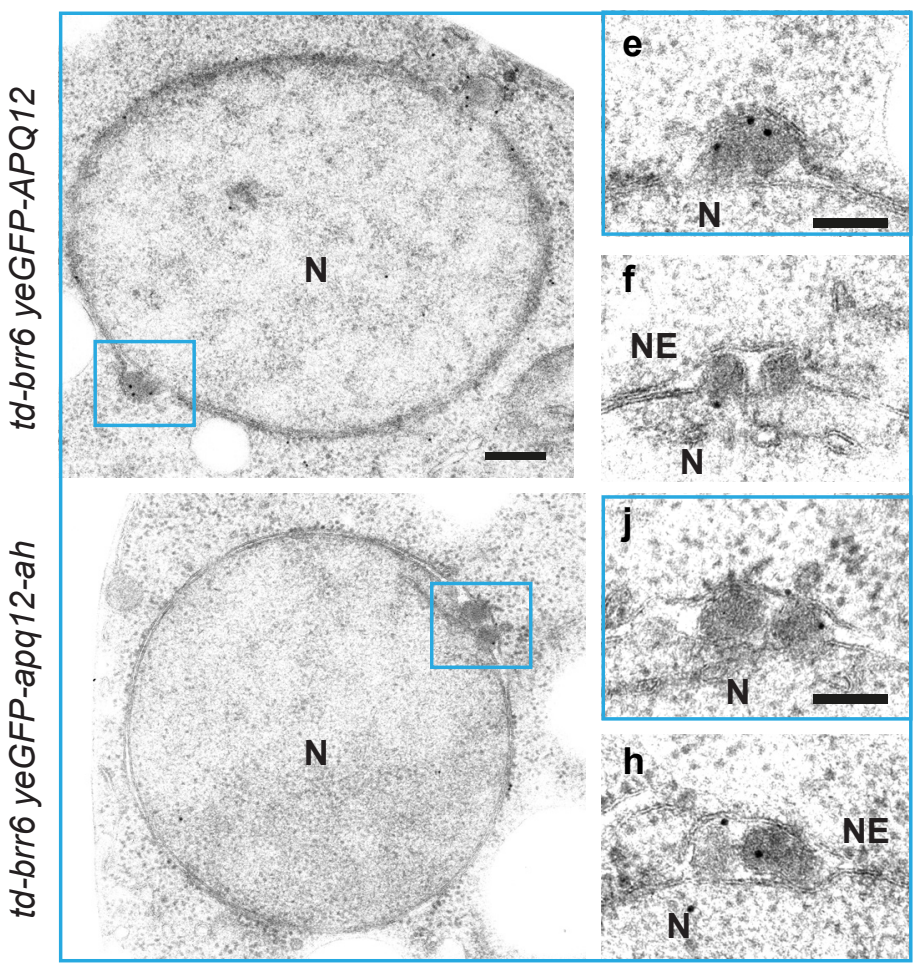

B

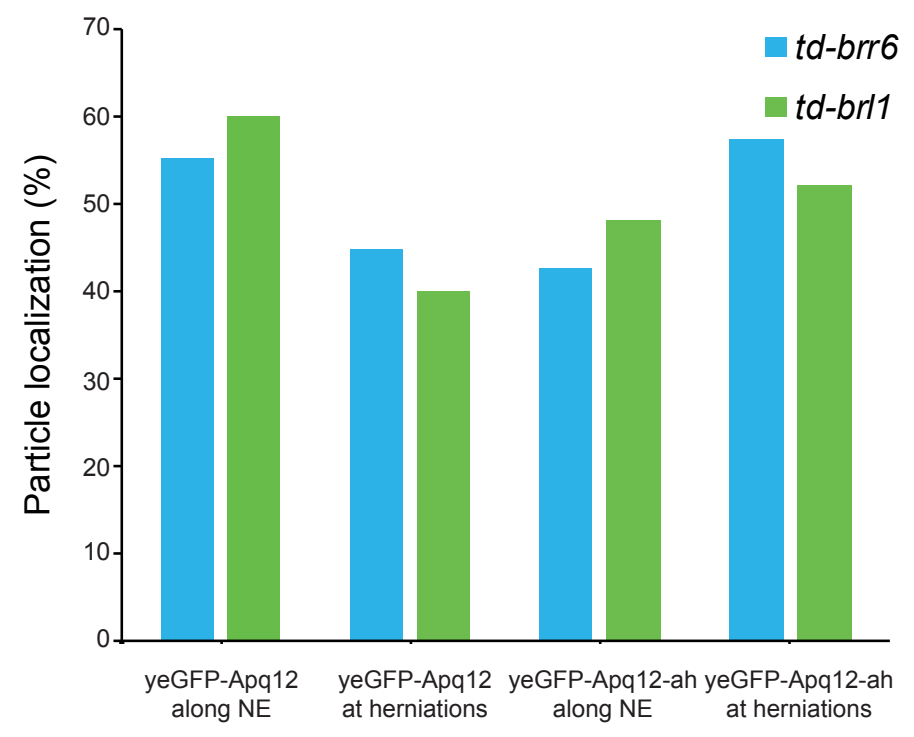

C

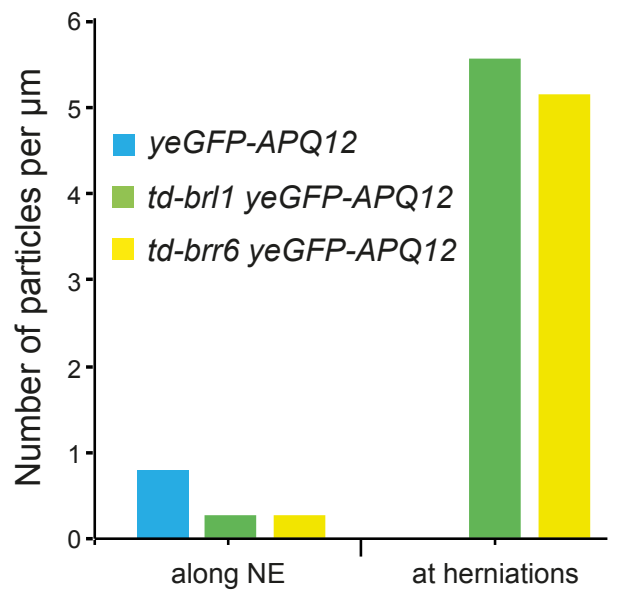


Table S1: Yeast strains and plasmids used in this study.

\begin{tabular}{|c|c|c|c|}
\hline Strain ID & Description & Source/Reference & Figure \\
\hline & ESM356-1 & & $2 A, 3 A$ \\
\hline YZW873 & 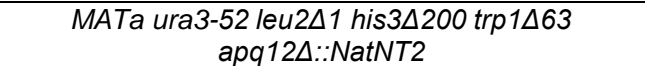 & This study & $2 \mathrm{~A}, 3 \mathrm{~B}, 9 \mathrm{~A}, \mathrm{~S} 2$ \\
\hline YZW952 & $\begin{array}{c}\text { MATa ura3-52 leu2 } \Delta 1 \text { his3 } \Delta 200 \text { trp1 } \Delta 63 \text { apq12- } \\
\text { ah-NatNT2 }\end{array}$ & This study & $2 \mathrm{~A}, 3 \mathrm{D}, 9 \mathrm{~A}, \mathrm{~S} 2$ \\
\hline YJV063 & $\begin{array}{c}\text { MATa ura3-52 leu2 } 11 \text { his } 3 \Delta 200 \text { trp1 } 1 \Delta 63 \\
\text { APQ12(F5DI6RV9N)::KanMX }\end{array}$ & This study & $2 \mathrm{~A}$ \\
\hline YZW895 & 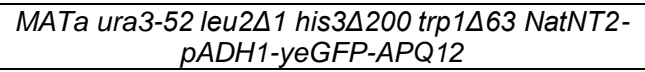 & This study & 2B, C, S1D \\
\hline AAK0032 & 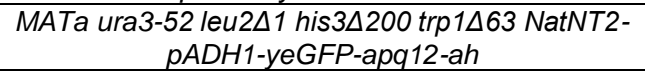 & This study & 2B, C, S1D \\
\hline AAK0018 & 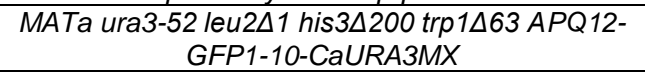 & This study & $2 \mathrm{D}$ \\
\hline & 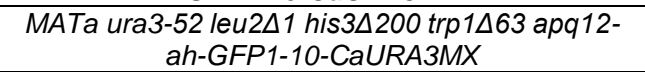 & This study & $2 \mathrm{D}$ \\
\hline AAK0019 & $\begin{array}{c}\text { MATa ura3-52 leu2 } 11 \text { his3 } 200 \text { trp1 } 163 \text { MPS3- } \\
\text { GFP1-10-CaURA3MX }\end{array}$ & This study & $2 \mathrm{D}$ \\
\hline AAK0033 & 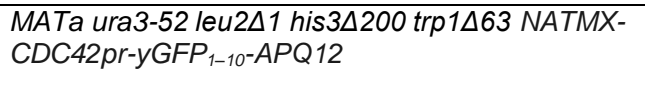 & This study & $2 \mathrm{D}$ \\
\hline AAK0034 & 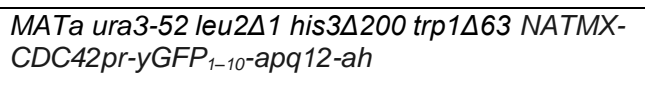 & This study & $2 \mathrm{D}$ \\
\hline YZW783 & 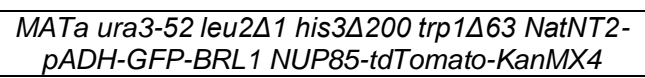 & This study & $4 C, 5 B, S 3 A, C, E$ \\
\hline YZW782 & 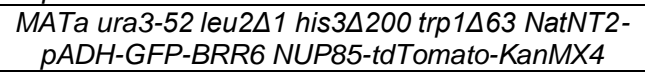 & This study & $4 F, 5 D, S 3 B, D, F$ \\
\hline YZW931 & 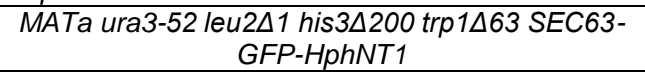 & This study & 41 \\
\hline YZW932 & 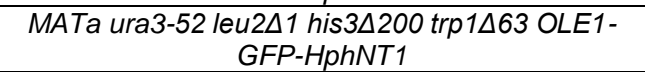 & This study & $4 \mathrm{~J}$ \\
\hline YZW738 & 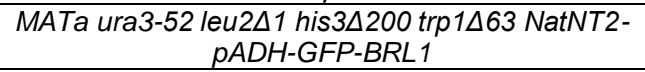 & This study & $6 \mathrm{C}, 7 \mathrm{~A}, \mathrm{D}$ \\
\hline & 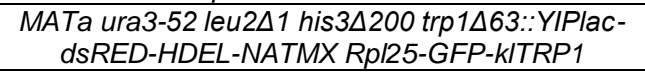 & This study & $6 \mathrm{C}$ \\
\hline AAK0011 & $\begin{array}{c}\text { GPY658 NatNT2-pADH1-yeGFP-APQ12, nup85- } \\
\text { tdTomato-HYG }\end{array}$ & This study & $8 \mathrm{~A}$ \\
\hline AAK0012 & $\begin{array}{c}\text { GPY658 KanMX6-pCup1-Ubi-R-DHFR(ts)-Myc- } \\
\text { BRR6 NatNT2-pADH1-yeGFP-APQ12, nup85- } \\
\text { tdTomato-hphMX4 }\end{array}$ & This study & $8 \mathrm{~A}, \mathrm{G}, \mathrm{S} 7 \mathrm{~A}$ \\
\hline AAK0013 & $\begin{array}{c}\text { GPY658 KanMX6-pCup1-Ubi-R-DHFR(ts)-Myc- } \\
\text { BRL1 NatNT2-pADH1-yeGFP-APQ12, nup85- } \\
\text { tdTomato-hphMX4 }\end{array}$ & This study & $8 \mathrm{~A}, \mathrm{G}, \mathrm{S} 7 \mathrm{~A}$ \\
\hline $\begin{array}{l}\text { YJV068 } \\
\end{array}$ & $\begin{array}{c}\text { GPY658 NatNT2-pADH1-yeGFP-apq12-ah, } \\
\text { nup85-tdTomato-HYG }\end{array}$ & This study & $8 \mathrm{D}$ \\
\hline YJV069 & $\begin{array}{c}\text { GPY658 KanMX6-pCup1-Ubi-R-DHFR(ts)-Myc- } \\
\text { BRR6 NatNT2-pADH1-yeGFP-apq12-ah, nup85- } \\
\text { tdTomato-hphMX4 }\end{array}$ & This study & $8 \mathrm{D}, \mathrm{G}, \mathrm{S} 7 \mathrm{~A}$ \\
\hline YJV070 & $\begin{array}{c}\text { GPY658 KanMX6-pCup1-Ubi-R-DHFR(ts)-Myc- } \\
\text { BRL1 NatNT2-pADH1-yeGFP-apq12-ah, nup85- } \\
\text { tdTomato-hphMX4 }\end{array}$ & This study & $8 \mathrm{D}, \mathrm{G}, \mathrm{S} 7 \mathrm{~A}$ \\
\hline YJV014 & 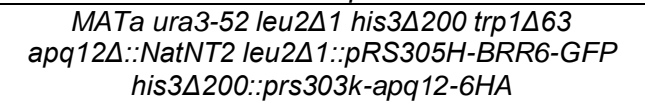 & This study & $9 \mathrm{C}, \mathrm{E}, \mathrm{F}$ \\
\hline YJV015 & 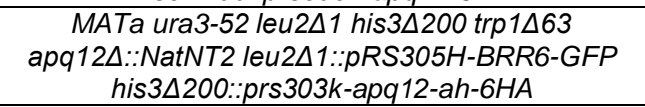 & This study & $9 \mathrm{C}, \mathrm{E}, \mathrm{F}$ \\
\hline YZW491 & $\begin{array}{c}\text { MATa ura3-52 leu2 } 21 \text { his3 } 200 \text { trp1 } 163 \text { BRL1- } \\
\text { GFP-HphNT1 }\end{array}$ & This study & $9 \mathrm{E}$ \\
\hline YJV073 & 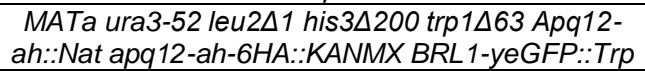 & This study & $9 \mathrm{E}$ \\
\hline YJV058 & 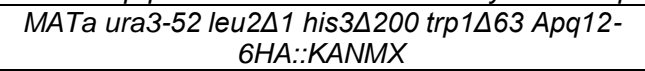 & This study & $9 \mathrm{~F}$ \\
\hline YJV059 & 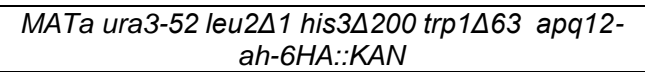 & This study & $9 \mathrm{~F}$ \\
\hline YZW902 & $\begin{array}{c}\text { MATa ura3-52 leu2 } \Delta 1 \text { his3 } \Delta 200 \text { trp1 } \triangle 63 \text { APQ12- } \\
\text { HBH-HphMX4 }\end{array}$ & This study & S1E \\
\hline
\end{tabular}




\begin{tabular}{|c|c|c|c|}
\hline YZW906 & $\begin{array}{c}\text { MATa ura3-52 his3 } 200 \text { trp1 } 163 \text { KanMX6-pGal1- } \\
\text { HBH-APQ12 }\end{array}$ & This study & S1E \\
\hline AAK0020 & 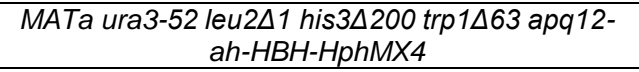 & This study & S1E \\
\hline AAK0021 & $\begin{array}{c}\text { MATa ura3-52 leu2 } \Delta 1 \text { his3 } \Delta 200 \text { trp1 } \Delta 63 \text { trp1- } \\
\text { pGAL-HBH-apq12-ah }\end{array}$ & This study & S1E \\
\hline YZW967 & 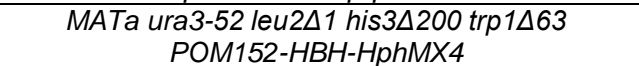 & This study & S1E \\
\hline YZW945 & $\begin{array}{c}\text { MATa ura3-52 his3 } 200 \text { trp1 } 163 \text { KanMX6-pGal1- } \\
\text { HBH-POM152 }\end{array}$ & This study & S1E \\
\hline YZW858 & 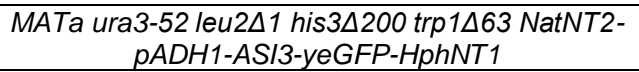 & Zhang et al., 2018 & S4A \\
\hline YZW292 & 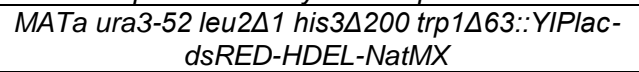 & This study & S4B \\
\hline YZW929 & 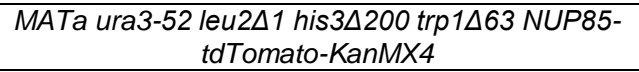 & This study & S5C \\
\hline Plasmid ID & Description & Source/Reference & \\
\hline & pFA6a-KanMX4 & M. Knop & \\
\hline pCM45 & pFA6a-tdTomato-KanMX6 & M. Knop & \\
\hline pCM49 & pFA6a-tdTomato-hphMX6 & M. Knop & \\
\hline pYM-N9 & ADH1promoter-yeGFP-natNT2 & Janke et al., 2004 & \\
\hline pKS134 & pFA6a-NatNT2 & M. Knop & \\
\hline pYM25 & yeGFP-hphNT1 & Janke et al., 2004 & \\
\hline pZW111 & p426Gal-APQ12-6His & this study & \\
\hline pZW112 & p425Gal-apq12-ah-6His & this study & \\
\hline Opi1 Q2-mCh & pRS316-CYC1prom-OPI1 Q2-mCh & this study & \\
\hline $\begin{array}{l}\text { NLS-Opi1 Q2- } \\
\text { mCh }\end{array}$ & pRS316-CYC1prom-NUP60(1-24)-OPI1 & Romanauska et al., 2018 & \\
\hline pZW114 & p426Gal-APQ12-yeGFP-8His & Romanauska et al., 2018 & \\
\hline pZW110 & p426Gal-APQ12 & this study & \\
\hline pAAK0010 & p415Gal-APQ12-6HIS & this study & \\
\hline pAAK0011 & p415Gal-apq12-ah-6HIS & this study & \\
\hline pSJ1256 & pFA6-link-yGFP ${ }_{1-10}-$ CaURA3MX & Smoyer et al., 2016 & \\
\hline pS1643 & pFA6-NATMX-CDC42pr-yGFP $P_{1-10}$ & Smoyer et al., 2016 & \\
\hline pSJ1321 & pRS315-NOP1pr-GFP $11-P U S 1$ & Smoyer et al., 2016 & \\
\hline \multirow[t]{2}{*}{ pSJ1568 } & pRS315-NOP1pr-GFP 11 -mCherry-SCS2TM & Smoyer et al., 2016 & \\
\hline & pFA6a-TRP1-pGal1-HBH & Tagwerker et al., 2006 & \\
\hline pZW37 & pFA6a-HBH-HphMX4-S3 & Zhang et al., 2018 & \\
\hline
\end{tabular}

